\title{
Optimizing the third-and-a-half post-Newtonian gravitational radiation-reaction force for numerical simulations
}

\author{
Guillaume Faye ${ }^{1, \text { * }}$ and Gerhard Schäfer ${ }^{1, \text { 田 }}$ \\ ${ }^{1}$ Theoretisch-Physikalisches Institut, Friedrich-Schiller-Universität, Max-Wien-Platz 1, 07743 Jena, Germany
}

(Dated: November 17, 2018)

\begin{abstract}
The gravitational radiation-reaction force acting on perfect fluids at 3.5 post-Newtonian order is cast into a form which is directly applicable to numerical simulations. Extensive use is made of metric-coefficient changes induced by functional coordinate transformations, of the continuity equation, as well as of the equations of motion. We also present an expression appropriate for numerical simulations of the radiation field causing the worked out reaction force.

PACS numbers: 04.25.-g
\end{abstract}

\section{INTRODUCTION}

The reaction force acting on isolated perfect fluids due to gravitational radiation emission has been expressed by means of Burke-Thorne-like potentials up to the $7 / 2$ post-Newtonian (3.5 PN) order, which corresponds to the seventh order in power of the inverse of the speed of light $c$ [1]. However, this particular form is apparently not convenient for computational purposes as it involves up to the seventh time derivative of multipole moments. A similar problem happens with the $1 \mathrm{PN}$ radiation field which causes the 3.5 PN reaction force, since up to four time derivatives do apply therein.

It is well known, e.g. see [2], that the order of time derivatives in the $2.5 \mathrm{PN}$ reaction force crucially depends on the chosen coordinate system. The influence of general changes of coordinates onto the metric coefficients of a many particle system has been worked out in e.g., [3], to the $2 \mathrm{PN}$ order. In generalizing this result to perfect fluids, combining it with the choice of a suitable set of variables, we shall be able to reduce the $3.5 \mathrm{PN}$ reaction force to a fifth time derivative object. Furthermore, applying the continuity equation and the equations of motion will result in expressions where only one time derivative remains left, which poses no problems for a numerical implementation. In this way we generalize a previous work by Rezzolla et al. [4 to the case of nonzero mass-multipole moments. We are then able to give the complete set of equations describing the fluid evolution up to $1 \mathrm{PN}+3.5 \mathrm{PN}$ order in a similar form as in a former paper by Blanchet, Damour and Schäfer [5]. In addition, we present the full explicit expression for the 1 $\mathrm{PN}$ radiation field, adapted to numerical simulations.

Though the formalism we propose limits to the case of adiabatic fluids, it may still be used in a large range of astrophysical applications. Particularly it provides a natural way to generalize the simulations of Oohara and Nakamura on the coalescence of binary neutron stars $[\underline{6}$ ]

\footnotetext{
*Electronic address: G.Faye@tpi.uni-jena.de

${ }^{\dagger}$ Electronic address: G.Schaefer@tpi.uni-jena.de
}

achieved at the $1 \mathrm{PN}$ conservative and 2.5 PN dissipative levels, by adding the $3.5 \mathrm{PN}$ contributions to the gravitational reaction force. Our equations are also appropriate to study the effect of the gravitational damping, including the mass quadrupole [], the mass octupole, as well as the current quadrupole (treated separately in paper [4]), on the evolution of fluid modes in rotating compact stars [8] in the case where the fluid viscosity is neglected. The role played by the bulk viscosity in reducing the Chandrasekhar-Friedman-Schutz instability [9, 10] may actually be significant, but it can be temporarily ignored, regarding the complexity of the problem. Our model of matter will eventually be improved in future works. Let us point out finally that the adiabaticity condition only means the entropy conservation of fluid particles along their trajectories, and does not impose any specific relation between energy, entropy and pressure. The equation of state linking these three variables can thus be freely chosen. Suitable choices [11, 12] permit to model for instance rather realistic stellar collapse processes within the present formalism.

\section{EFFECT OF A GAUGE TRANSFORMATION ON A METRIC}

In order to determine the coordinate system that minimizes the order of time derivatives appearing in the reaction force, we need to know the specific effect of a general change of coordinates on the functional form of the metric. More precisely, we must extend the "contact transformation" investigated in paper [3] for systems of point-like particles to the case where the gravitational field is generated by a continuous distribution of matter. To determine the order at which we need to operate, we notice that the parts of the metric we are interested in contribute to the $1 \mathrm{PN}$ acceleration, as well as to the 2.5 and 3.5 PN dissipative dynamics. Products of terms corresponding to the $1 \mathrm{PN}$ and $2.5 \mathrm{PN}$ level in the equations of motion cannot be neglected since they affect the 3.5 PN evolution. Therefore, the gauge transformation must be a priori investigated up to the quadratic order. However, we shall see that the linear approximation is suf- 
ficient provided the difference between the old and new coordinates is given in terms of the new variables. Still, some formulas, like formula (4.2) which shows how two successive gauge transformations of order $1 \mathrm{PN}$ and 2.5 +3.5 differs from the transformation induced by the sum of the associated change of coordinates, can only be obtained within the quadratic approximation. Now, rather than limiting ourselves to the quadratic level, we shall give for completeness most of the relations presented in this section to arbitrary high orders. The resulting expressions will be longer, but more general and not fundamentally more complicated. They may be useful for future works, although not strictly required in the present paper. The reader solely interested in the application to the actual problem of eliminating time derivatives from the $3.5 \mathrm{PN}$ reaction force may skip this section. The reader exclusively interested in the final result may go directly to the end of section IV (equations 4.31).

In a given coordinate grid, the metric $g_{\mu \nu}$ (Greek indices run from 0 to 3 , and Latin indices from 1 to 3 ) is a function of the coordinate $x^{\alpha}=\left(c t=x^{0}, \mathbf{x}=\right.$ $x^{i}$ ) and depends functionally on certain matter fields $X_{1}, X_{2}, \ldots, X_{k}, \ldots$ In the case of barotropic fluids, for instance, they can be the mass density $\rho$ and the 4 -velocity field $u^{\mu}$ normalized to unity $\left(u^{\mu} u_{\mu}=-1\right)$, or the baryonic mass density $\rho_{*}$ and the linear 3 -momentum density $M_{i}$, or other sets of relevant variables. We have:

$$
g_{\mu \nu}=g_{\mu \nu}\left(x^{\alpha}, X_{1}\left(y^{\alpha}\right), X_{2}\left(y^{\alpha}\right), \ldots, X_{k}\left(y^{\alpha}\right)\right)
$$

in short form, $g_{\mu \nu}=g_{\mu \nu}\left(x^{\alpha}, X_{A}\left(y^{\alpha}\right)\right)$. In our notation, the variables $x^{\alpha}$ contain the entire dependence on the coordinates, whereas $y^{\alpha}$ are seen as mere "dummy" quantities. The fields $X_{A}\left(y^{\alpha}\right)$ are in fact themselves functionals of the components of the stress energy-tensor and of the metric, $X_{A}\left(y^{\alpha}\right)=F_{A}\left(y^{\alpha}, T^{\alpha \beta}, g_{\alpha \beta}\right)$, even if it is not indicated explicitly for simplicity. The important point is that a coordinate transformation, which is passive by essence, affects the function $X_{A}=X_{A}\left(y^{\alpha}\right)$.

Let us consider now the (exact) change of coordinates

$$
x^{\alpha}=x^{\prime \alpha}+\varepsilon_{x^{\prime}}^{\alpha}\left(x^{\prime \beta}, X_{A}^{\prime}\left(y^{\beta}\right)\right)
$$

where $\varepsilon_{x^{\prime}}^{\alpha}$ is a function of $x^{\prime \beta}$ and a functional of the fields $X_{A}^{\prime}\left(y^{\beta}\right)$. Such a transformation acts simultaneously on the coordinates, on the metric and on the fields $X_{A}\left(y^{\alpha}\right)$. As a consequence, the new components of $g_{\mu \nu}^{\prime}$ are related to the old ones by

$$
\begin{aligned}
g_{\mu \nu}^{\prime}\left(x^{\prime \alpha}, X_{A}^{\prime}\left(y^{\alpha}\right)\right) & =g_{\mu \nu}\left(x^{\alpha}, X_{A}\left(y^{\alpha}\right)\right) \\
+ & 2 g_{\lambda(\mu}\left(x^{\underline{\alpha}}, X_{A}\left(y^{\underline{\alpha}}\right)\right) \partial_{\nu)}^{\prime} \varepsilon_{x^{\prime}}^{\lambda}\left(x^{\prime \beta}, X_{A}^{\prime}\left(y^{\beta}\right)\right) \\
+ & g_{\lambda \rho}\left(x^{\alpha}, X_{A}\left(y^{\alpha}\right)\right) \partial_{\mu}^{\prime} \varepsilon_{x^{\prime}}^{\lambda}\left(x^{\prime \beta}, X_{A}^{\prime}\left(y^{\beta}\right)\right) \\
& \times \partial_{\nu}^{\prime} \varepsilon_{x^{\prime}}^{\rho}\left(x^{\prime \gamma}, X_{A}^{\prime}\left(y^{\gamma}\right)\right),
\end{aligned}
$$

where $\partial_{\lambda}^{\prime}$ represents the partial derivative with respect to $x^{\prime \lambda}$, while the brackets around non-underlined indices mean their symmetrization. The fields $X_{A}^{\prime}\left(y^{\alpha}\right)$ entering the argument of $g_{\mu \nu}^{\prime} \operatorname{read} X_{A}^{\prime}\left(y^{\alpha}\right)=F_{A}\left(y^{\alpha}, T^{\prime \alpha \beta}, g_{\alpha \beta}^{\prime}\right)$. Since the dependence on $T^{\prime \alpha \beta}\left(z^{\gamma}\right)$ and $g_{\alpha \beta}^{\prime}\left(z^{\gamma}\right)$ is purely functional, the position variables $z^{\gamma}$ appearing there must be regarded as dummy, but their other arguments are combinations of tensor components referring to the current coordinate system: $T^{\prime \alpha \beta}=T^{\alpha \beta}\left(z^{\gamma}, g_{\gamma \delta}^{\prime}, \ldots\right)$ and $g_{\alpha \beta}^{\prime}=g_{\alpha \beta}^{\prime}\left(z^{\gamma}, X_{B}^{\prime}\right)$. In the post-Newtonian framework, the computation of $g_{\mu \nu}^{\prime}$ is actually restricted to a finite order in powers of $\varepsilon_{x^{\prime}}^{\alpha}$ if we assume that the latter quantity is of order of the inverse of the speed of light $c$ or higher, namely $\varepsilon_{x^{\prime}}^{\alpha}=\mathcal{O}(1 / c)$. Therefore, when the function $g_{\mu \nu}\left(x^{\alpha}, X_{A}\left(y^{\alpha}\right)\right)$ is known, the dependence of $g_{\mu \nu}^{\prime}$ on $x^{\prime \alpha}$ can be obtained explicitly by expanding the righthand side of equation (2.1) in powers of $\varepsilon_{x^{\prime}}^{\alpha}=x^{\alpha}-x^{\prime \alpha}$ within the required precision. Since the order of expansion can be arbitrarily high, we may symbolically write

$$
\begin{aligned}
g_{\mu \nu}^{\prime}\left(x^{\prime \alpha}, X_{A}^{\prime}\left(y^{\alpha}\right)\right)= & g_{\mu \nu}\left(x^{\prime \alpha}, X_{A}\left(y^{\alpha}\right)\right)+\varepsilon_{x^{\prime}}^{\lambda}\left(x^{\prime \alpha}, X_{A}^{\prime}\left(y^{\alpha}\right)\right) \partial_{\lambda}^{\prime} g_{\mu \nu}\left(x^{\prime \beta}, X_{A}\left(y^{\beta}\right)\right)+2 g_{\lambda(\mu}\left(x^{\prime \underline{\alpha}}, X_{A}\left(y^{\underline{\alpha}}\right)\right) \partial_{\nu)}^{\prime} \varepsilon_{x^{\prime}}^{\lambda}\left(x^{\prime \beta}, X_{A}^{\prime}\left(y^{\beta}\right)\right) \\
& +\sum_{k=0}^{+\infty} \frac{1}{k !}\left(\frac{\varepsilon_{x^{\prime}}^{\lambda_{1} \ldots \lambda_{k} \lambda \rho}}{(k+1)(k+2)}\left(x^{\prime \alpha}, X_{A}^{\prime}\left(y^{\alpha}\right)\right) \partial_{\lambda_{1} \ldots \lambda_{k} \lambda \rho}^{\prime} g_{\mu \nu}\left(x^{\prime \beta}, X_{A}\left(y^{\beta}\right)\right)\right. \\
& +\frac{2}{k+1} \varepsilon_{x^{\prime}}^{\lambda_{1} \ldots \lambda_{k} \rho}\left(x^{\prime \alpha}, X_{A}^{\prime}\left(y^{\alpha}\right)\right) \partial_{\lambda_{1} \ldots \lambda_{k} \rho}^{\prime} g_{\lambda(\mu}\left(x^{\prime} \underline{\beta}, X_{A}\left(y^{\underline{\beta}}\right)\right) \partial_{\nu)}^{\prime} \varepsilon_{x^{\prime}}^{\lambda}\left(x^{\prime \gamma}, X_{A}^{\prime}\left(y^{\gamma}\right)\right) \\
& \left.+\varepsilon_{x^{\prime}}^{\lambda_{1} \ldots \lambda_{k}}\left(x^{\prime \alpha}, X_{A}^{\prime}\left(y^{\alpha}\right)\right) \partial_{\lambda_{1} \ldots \lambda_{k}} g_{\lambda \rho}\left(x^{\prime \beta}, X_{A}\left(y^{\beta}\right)\right) \partial_{\mu}^{\prime} \varepsilon_{x^{\prime}}^{\lambda} \partial_{\nu}^{\prime} \varepsilon_{x^{\prime}}^{\rho}\left(x^{\prime \gamma}, X_{A}^{\prime}\left(y^{\gamma}\right)\right)\right) .
\end{aligned}
$$

Note that according to the Einstein summation convention, repeated indices are summed over all their possible values; $\varepsilon_{x^{\prime}}^{\lambda_{1} \ldots \lambda_{k}}$ is a short form for the product $\varepsilon_{x^{\prime}}^{\lambda_{1}} \ldots \varepsilon_{x^{\prime}}^{\lambda_{k}}$, and $\partial_{\lambda_{1} \ldots \lambda_{k}}^{\prime}$ stands for $\partial_{\lambda_{1}}^{\prime} \ldots \partial_{\lambda_{k}}^{\prime}$. Again, the infinite sum must be regarded as a formal series and does not need to converge. It has to be truncated at a given order consistently with the general approximation scheme. There only remains, at this stage, to express the metric components appearing in the second member of equation (2.2) with the help of the new fields $X_{A}^{\prime}\left(y^{\alpha}\right)$ rather than the 
old $X_{A}\left(y^{\alpha}\right)$. It can be most easily performed by using the concept of Fréchet derivative. Let us consider that $g_{\mu \nu}\left(x^{\alpha}, X_{A}\left(y^{\alpha}\right)\right)$ is a function of the field variable $X_{1}$, on the affine space $\mathbb{R}$, having its domain on an affine space $\mathcal{E}_{1}$ (for instance, the space of smooth bounded real function operating on $\mathbb{R}^{4}$, embedded with a norm, say ||$\left._{\infty}\right)$. By definition, the Fréchet derivative of $g_{\mu \nu}\left(x^{\alpha}, X_{A}\left(y^{\alpha}\right)\right)$ with respect to $X_{1}$ is, when it exists, the continuous linear form, on the vector space associated to $\mathcal{E}_{1}$, that satisfies

$$
\begin{aligned}
& g_{\mu \nu}\left(x^{\alpha}, X_{1}^{\prime}\left(y^{\alpha}\right), X_{A>1}\left(y^{\alpha}\right)\right)-g_{\mu \nu}\left(x^{\alpha}, X_{A}\left(y^{\alpha}\right)\right) \\
& =D_{X_{1}}\left[g_{\mu \nu}\left(x^{\alpha}, X_{A}\left(y^{\alpha}\right)\right)\right] \cdot \delta_{\varepsilon_{x^{\prime}}}^{*} X_{1}\left(y^{\beta}\right)+o\left(\left|\delta_{\varepsilon_{x^{\prime}}}^{*} X_{1}\left(y^{\beta}\right)\right|_{\infty}^{2}\right)
\end{aligned}
$$

for any infinitesimal variation $\delta_{\varepsilon_{x^{\prime}}}^{*} X_{1}\left(y^{\beta}\right) \equiv X_{1}^{\prime}\left(y^{\beta}\right)-$ $X_{1}\left(y^{\beta}\right) \in \mathcal{E}_{1}$. Following usual notations, we have inserted a dot immediately before the variation $\delta_{\varepsilon_{x^{\prime}}}^{*} X_{1}\left(y^{\beta}\right)$ to indicate the action of the form resulting from the derivation. Denoting by $\mathcal{L}\left(\mathcal{E}_{1}\right)$ the vector space of the continu- ous linear form on $\mathcal{E}_{1}$, we have $D_{X_{1}}\left[g_{\mu \nu}\left(x^{\alpha}, X_{A}\left(y^{\alpha}\right)\right)\right] \in$ $\mathcal{L}\left(\mathcal{E}_{1}\right)$. Now, $\mathcal{L}\left(\mathcal{E}_{1}\right)$ can be itself embedded with a structure of affine space as the real number set $\mathbb{R}$, so that we can define the Fréchet derivative of functions $f$ : $\mathcal{E}_{1} \rightarrow \mathcal{L}\left(\mathcal{E}_{1}\right)$ in a similar way as before. The bilinear form $D_{X_{B}}\left[D_{X_{1}}\left[g_{\mu \nu}\left(x^{\alpha}, X_{A}\left(y^{\alpha}\right)\right)\right]\right]$ is said to be the second Fréchet derivative of $g_{\mu \nu}\left(x^{\alpha}, X_{A}\left(y^{\alpha}\right)\right)$ with respect to $X_{B}$ and $X_{1}$, and similarly for higher orders. As the derivative of the difference $g_{\mu \nu}\left(x^{\alpha}, X_{1}^{\prime}\left(y^{\alpha}\right), X_{A>1}\left(y^{\alpha}\right)\right)-$ $g_{\mu \nu}\left(x^{\alpha}, X_{A}\left(y^{\alpha}\right)\right)$ with respect to $x^{\beta}$ or $X_{B}\left(y^{\beta}\right)$ is equal to the difference of the derivatives, the operator $D_{X_{1} \ldots X_{A_{k}}}$ $(k \in \mathbb{N})$ is evidently symmetric and commutes with the space-time derivatives $\partial_{\lambda_{1} \ldots \lambda_{k}}$. We are then in order to achieve our goal of removing the dependence of the metric on $X_{A}\left(y^{\beta}\right)$ by expanding $g_{\mu \nu}^{\prime}\left(x^{\alpha}, X_{A}^{\prime}\left(y^{\alpha}\right)-\delta_{\varepsilon_{x^{\prime}}}^{*} X_{A}\left(y^{\alpha}\right)\right)$ around the fields $X_{A}^{\prime}\left(y^{\alpha}\right)$ after a generalized Taylor theorem [13]. This yields the fundamental relation:

$$
\begin{gathered}
\delta_{\varepsilon_{x^{\prime}}}^{*} g_{\mu \nu} \equiv g_{\mu \nu}^{\prime}-g_{\mu \nu}=\sum_{l=1}^{+\infty} \frac{(-1)^{l}}{l !} D_{X_{B_{1}} \ldots X_{B_{l}}}\left[g_{\mu \nu}\right] .\left(\delta_{\varepsilon_{x^{\prime}}}^{*} X_{B_{1}}, \ldots, \delta_{\varepsilon_{x^{\prime}}}^{*} X_{B_{l}}\right) \\
+\sum_{l=0}^{+\infty} \frac{(-1)^{l}}{l !}\left(\varepsilon_{x^{\prime}}^{\lambda} \partial_{\lambda} D_{X_{B_{1}} \ldots X_{B_{l}}}\left[g_{\mu \nu}\right] \cdot\left(\delta_{\varepsilon_{x^{\prime}}}^{*} X_{B_{1}}, \ldots, \delta_{\varepsilon_{x^{\prime}}}^{*} X_{B_{l}}\right)+2 D_{X_{B_{1}} \ldots X_{B_{l}}}\left[g_{\lambda(\mu}\right] \cdot\left(\delta_{\varepsilon_{x^{\prime}}}^{*} X_{B_{1}}, \ldots, \delta_{\varepsilon_{x^{\prime}}}^{*}, X_{B_{l}}\right) \partial_{\nu)} \varepsilon_{x^{\prime}}^{\lambda}\right) \\
+\sum_{k, l \geq 0} \frac{(-1)^{l}}{k ! l !}\left(\frac{\varepsilon_{x^{\prime}}^{\lambda_{1} \ldots \lambda_{k} \lambda \rho}}{(k+1)(k+2)} \partial_{\lambda_{1} \ldots \lambda_{k} \lambda \rho} D_{X_{B_{1}} \ldots X_{B_{l}}}\left[g_{\mu \nu}\right] \cdot\left(\delta_{\varepsilon_{x^{\prime}}}^{*} X_{B_{1}}, \ldots, \delta_{\varepsilon_{x^{\prime}}}^{*} X_{B_{l}}\right)\right. \\
\quad+\frac{2}{k+1} \varepsilon_{x^{\prime}}^{\lambda_{1} \ldots \lambda_{k} \rho} \partial_{\lambda_{1} \ldots \lambda_{k} \rho} D_{X_{B_{1}} \ldots X_{B_{l}}}\left[g_{\lambda(\mu}\right] \cdot\left(\delta_{\varepsilon_{x^{\prime}}}^{*} X_{B_{1}}, \ldots, \delta_{\varepsilon_{x^{\prime}}}^{*}, X_{B_{l}}\right) \partial_{\nu)} \varepsilon_{x^{\prime}}^{\lambda} \\
\left.+\varepsilon_{x^{\prime}}^{\lambda_{1} \ldots \lambda_{k}} \partial_{\lambda_{1} \ldots \lambda_{k}} D_{X_{B_{1}} \ldots X_{B_{l}}}\left[g_{\lambda \rho}\right] \cdot\left(\delta_{\varepsilon_{x^{\prime}}}^{*}, X_{B_{1}}, \ldots, \delta_{\varepsilon_{x^{\prime}}}^{*} X_{B_{l}}\right) \partial_{\mu} \varepsilon_{x^{\prime}}^{\lambda} \partial_{\nu} \varepsilon_{x^{\prime}}^{\rho}\right)
\end{gathered}
$$

where $\left(\delta_{\varepsilon_{x^{\prime}}}^{*}, X_{B_{1}}, \ldots, \delta_{\varepsilon_{x^{\prime}}}^{*} X_{B_{l}}\right)$ denotes the vector to which the $l$-form $D_{X_{B_{1}} \ldots X_{B_{l}}}\left[g_{\mu \nu}\right]$ applies. It tends toward zero as $c$ goes to infinity since $X_{B}^{\prime}$ and $X_{B}$ coincide when $\varepsilon_{x^{\prime}}^{\alpha}=0$. As initially required, all quantities entering the above expression depend implicitly on the same variables, say $x^{\alpha}$ and $X_{A}$. In the grid $x^{\prime \alpha}=x^{\alpha}-\varepsilon_{x^{\prime}}^{\alpha}\left(x^{\prime \beta}, X_{B}^{\prime}\right)$, the new components of the metric are given, at last, by the function $g_{\mu \nu}^{\prime}\left(x^{\prime \alpha}, X_{A}^{\prime}\right)=g_{\mu \nu}\left(x^{\prime \alpha}, X_{A}^{\prime}\right)+\delta_{\varepsilon_{x^{\prime}}}^{*} g_{\mu \nu}\left(x^{\prime \alpha}, X_{A}^{\prime}\right)$. In the same way, after a further transformation $x^{\prime \alpha}=$ $x^{\prime \prime \alpha}+\varepsilon_{x^{\prime \prime}}^{\prime \alpha}\left(x^{\prime \prime \beta}, X_{B}^{\prime \prime}\right)$, the metric components become $g_{\mu \nu}^{\prime \prime}\left(x^{\prime \prime \alpha}, X_{A}^{\prime \prime}\right)=g_{\mu \nu}^{\prime}\left(x^{\prime \prime \alpha}, X_{A}^{\prime \prime}\right)+\delta_{\varepsilon_{x^{\prime \prime}}^{\prime}}^{*} g_{\mu \nu}^{\prime}\left(x^{\prime \prime \alpha}, X_{A}^{\prime \prime}\right)$. This proves the interesting relation:

$$
\begin{aligned}
x^{\mu} & =x^{\prime \prime \mu}+\varepsilon_{x^{\prime \prime}}^{\prime \mu}\left(x^{\prime \prime \alpha}, X_{A}^{\prime \prime}\right) \\
+ & \varepsilon_{x^{\prime}}^{\mu}\left(x^{\prime \prime \alpha}+\varepsilon^{\prime \alpha}{ }_{x^{\prime \prime}}\left(x^{\prime \prime \beta}, X_{B}^{\prime \prime}\right), X_{A}^{\prime \prime}-\delta_{\varepsilon_{x^{\prime \prime}}^{\prime}}^{*} X_{A}^{\prime}\right) \\
& =x^{\prime \prime \mu}+\varepsilon_{x^{\prime \prime}}^{\prime \mu}\left(x^{\prime \prime \alpha}, X_{A}^{\prime \prime}\right)+\varepsilon_{x^{\prime}}^{\mu}\left(x^{\prime \prime \alpha}, X_{A}^{\prime \prime}\right)
\end{aligned}
$$

$$
\begin{array}{r}
+\sum_{\substack{l, k \geq 0 \\
l+k \geq 1}} \frac{(-1)^{l}}{k ! l !} \varepsilon^{l} \varepsilon_{x^{\prime \prime} \ldots \lambda_{k}}^{\lambda_{1} \ldots \lambda_{\lambda_{1} \ldots \lambda_{k}}^{\prime \prime}} D_{X_{B_{1}}^{\prime \prime} \ldots X_{B_{l}}^{\prime \prime}}^{\prime \prime}\left[\varepsilon_{x^{\prime}}^{\mu}\left(x^{\prime \prime \alpha}, X_{A}^{\prime \prime}\right)\right] \\
\cdot\left(\delta_{\varepsilon_{x^{\prime \prime}}^{\prime}}^{*} X_{B_{1}}^{\prime}, \ldots, \delta_{\varepsilon_{x^{\prime \prime}}^{\prime}}^{*} X_{B_{l}}^{\prime}\right) .
\end{array}
$$

The preceding transformation between the initial and final coordinates defines a function $\varepsilon_{x^{\prime \prime}}^{\mu}$ by $x^{\mu}=x^{\prime \prime}+\varepsilon_{x^{\prime \prime}}^{\mu}$. Equation (2.4) shows that this function is not the mere superposition of $\varepsilon_{x^{\prime}}^{\mu}$ and $\varepsilon_{x^{\prime \prime}}^{\prime \mu}$ but also involves non-linear contributions. We already know that the metric components $g_{\mu \nu}^{\prime}$ obtained after the first coordinate change are given by $g_{\mu \nu}^{\prime}=g_{\mu \nu}+\delta_{\varepsilon_{x^{\prime}}}^{*} g_{\mu \nu}$. The ten functions $g_{\mu \nu}^{\prime \prime}$ can be similarly deduced from $g_{\mu \nu}^{\prime}: g_{\mu \nu}^{\prime \prime}=g_{\mu \nu}^{\prime}+\delta_{\varepsilon_{x^{\prime \prime}}^{\prime}}^{*} g_{\mu \nu}^{\prime}$. On the other hand, as the grid $\left\{x^{\prime \prime \mu}\right\}$ is related to the old coordinate system $\left\{x^{\mu}\right\}$ by $x^{\alpha}=x^{\prime \prime \alpha}+\varepsilon_{x^{\prime \prime}}^{\alpha}$, we have $g_{\mu \nu}^{\prime \prime}=g_{\mu \nu}+\delta_{\varepsilon_{x^{\prime \prime}}}^{*} g_{\mu \nu}$ (which can be checked explicitly). Finally, the quantities $g_{\mu \nu}^{\prime \prime \prime}=g_{\mu \nu}+\delta_{\varepsilon_{x^{\prime}}^{\mu}+\varepsilon_{x^{\prime \prime}}^{\prime \prime}}^{*} g_{\mu \nu}$ represent the components of the metric in the coordinates system $\left\{x^{\prime \prime \prime}{ }^{\alpha}\right\}$ such that $x^{\alpha}=x^{\prime \prime \prime} \alpha+\varepsilon_{x^{\prime}}^{\alpha}\left(x^{\prime \prime \prime}{ }^{\beta}, X_{A}^{\prime \prime \prime}\left(y^{\beta}\right)\right)+$ 
$\varepsilon_{x^{\prime \prime}}^{\alpha}\left(x^{\prime \prime \prime} \beta, X_{A}^{\prime \prime \prime}\left(y^{\beta}\right)\right)$. They refer to the same gravitational field as $g_{\mu \nu}^{\prime}$, or $g_{\mu \nu}^{\prime \prime}$. In all what precedes, the new coordinates are defined by implicit equations for given $\varepsilon_{x^{\prime}}^{\alpha}$ and $\varepsilon_{x^{\prime \prime}}^{\prime \alpha}$, e.g. $x^{\prime \alpha}=x^{\alpha}-\varepsilon_{x^{\prime}}^{\alpha}\left(x^{\prime \beta}, X_{A}^{\prime}\left(y^{\beta}\right)\right)$. However, they can be formally inverted by applying recursively the Taylor formula and rearranging the summation,

$$
\begin{aligned}
& x^{\prime \alpha}=x^{\alpha}-\sum_{n, m \geq 0}^{+\infty} \frac{(-1)^{n}}{m !} \sum_{s=1}^{+\infty} \sum_{\substack{k_{1}+\ldots+k_{s}=n \\
k_{i} \geq 1}} \frac{1}{k_{1} ! \ldots k_{s} !} D\left[\varepsilon^{\lambda_{1}^{(n)} \ldots \lambda_{k_{n}}^{(n)}} \partial_{\lambda_{1}^{(n)} \ldots \lambda_{k_{n}}^{(n)}} \times\right.
\end{aligned}
$$

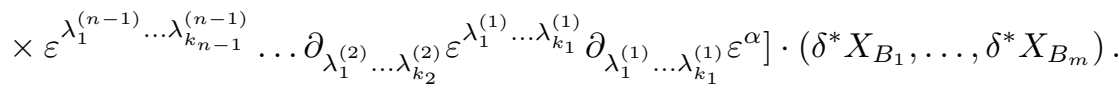

In most of applications, the post-Newtonian metric components show the structure of a sum of products of elementary factors, each of them being itself a $n$-tuple spatial integral of a given function $f\left(\mathbf{y}, \mathbf{z}_{1}, \ldots, \mathbf{z}_{n-1}, t, X_{A}(\mathbf{y}, c t)\right)$ of the coordinates and the fields. With the help of the Leibniz rule, the Fréchet derivative $D_{X_{A}}\left[g_{\mu \nu}\left(x^{\alpha}, X_{B}\left(y^{\alpha}\right)\right)\right]$ can be written as a sum of terms matching the pattern:

$$
F\left(x^{\alpha}, X_{C}\left(y^{\alpha}\right)\right) D_{X_{A}}\left[\int d^{3} \mathbf{y}_{1} \ldots d^{3} \mathbf{y}_{n} f\left(\mathbf{y}_{\mathbf{1}}, \mathbf{y}_{\mathbf{2}}, \ldots, \mathbf{y}_{\mathbf{n}}, t, X_{D}\left(\mathbf{y}_{\mathbf{1}}, c t\right)\right)\right] \cdot \delta_{\varepsilon_{x^{\prime}}}^{*} X_{B}
$$

where $F\left(x^{\alpha}, X_{C}\left(y^{\alpha}\right)\right)$ is a function of $x^{\alpha}$ and a functional of $\left.X_{C}\left(y^{\alpha}\right)\right)$. After the definition of $D_{X_{A}}$, the latter expression also reads:

$$
\int d^{3} \mathbf{y}_{1} \delta_{\varepsilon_{x^{\prime}}}^{*} X_{A} \int d^{3} \mathbf{y}_{2} \ldots d^{3} \mathbf{y}_{n} F\left(x^{\alpha}, X_{C}\left(y^{\alpha}\right)\right) \partial_{X_{A}} f\left(\mathbf{y}_{\mathbf{1}}, \mathbf{y}_{\mathbf{2}}, \ldots, \mathbf{y}_{\mathbf{n}}, t, X_{D}\left(\mathbf{y}_{\mathbf{1}}, c t\right)\right)
$$

From what we conclude that $D_{X_{A}}\left[g_{\mu \nu}\left(x^{\alpha}, X_{B}\left(y^{\alpha}\right)\right)\right] . \delta_{\varepsilon_{x^{\prime}}}^{*} X_{A}$ admits an integral representation of the type $\int d^{3} \mathbf{y} \delta_{\varepsilon_{x^{\prime}}}^{*} X_{A}\left(\delta g_{\mu \nu} / \delta X_{A}\right)$. The so-defined quantity $\delta g_{\mu \nu} / \delta X_{A}$ is known as the first functional derivative of $g_{\mu \nu}\left(x^{\alpha}, X_{B}\left(y^{\alpha}\right)\right)$ with respect to $X_{A}$. It is also possible to introduce the second functional derivative $\delta^{2} g_{\mu \nu} / \delta X_{A} \delta X_{B}$. By definition,

$$
\int d^{3} \mathbf{y}_{1} d^{3} \mathbf{y}_{2} \delta_{\varepsilon_{x^{\prime}}}^{*} X_{B}\left(\mathbf{y}_{1}, c t\right) \delta_{\varepsilon_{x^{\prime}}}^{*} X_{C}\left(\mathbf{y}_{2}, c t\right) \frac{\delta^{2} g_{\mu \nu}}{\delta_{\varepsilon_{x^{\prime}}}^{*} X_{B} \delta_{\varepsilon_{x^{\prime}}}^{*} X_{C}} \equiv D_{X_{B} X_{C}}\left[g_{\mu \nu}\left(x^{\alpha}, X_{A}\left(y^{\alpha}\right)\right)\right] \cdot\left(\delta_{\varepsilon_{x^{\prime}}}^{*} X_{B}\left(y^{\beta}\right), \delta_{\varepsilon_{x^{\prime}}}^{*} X_{C}\left(y^{\gamma}\right)\right) .
$$

The case where the non-linear terms occurring in (2.3) do not contribute to $\delta_{\varepsilon_{x^{\prime}}}^{*} g_{\mu \nu}$ (or at least to the order of $g_{\mu \nu}$ we are interested in) is of particular importance. This happens when all products involving two factors equal to $\delta_{\varepsilon_{x^{\prime}}}^{*} X_{B}$ or $\varepsilon_{x^{\prime}}$ as well as a space or time derivative of the metric $\partial g$ can be neglected. Hence, the $n$ $\mathrm{PN}$ transformation law is linear whenever $\mathcal{O}\left(1 / c^{n+1}\right)=$ $\mathcal{O}\left(\varepsilon_{x^{\prime}}^{2} \partial g\right)+\mathcal{O}\left(\varepsilon_{x^{\prime}} \delta_{\varepsilon_{x^{\prime}}}^{*} X \partial g\right)+\mathcal{O}\left(\left(\delta_{\varepsilon_{x^{\prime}}}^{*} X\right)^{2} \partial g\right)$, and equation (2.3) reduces then to:

$$
\begin{aligned}
\delta_{\varepsilon_{x^{\prime}}}^{*} g_{\mu \nu}=-D_{X_{B}}\left[g_{\mu \nu}\right] & \cdot \delta_{\varepsilon_{x^{\prime}}}^{*} X_{B} \\
& +\varepsilon_{x^{\prime}}^{\lambda} \partial_{\lambda} g_{\mu \nu}+2 g_{\lambda(\mu} \partial_{\nu)} \varepsilon_{x^{\prime}}^{\lambda}
\end{aligned}
$$

As a check, let us specify relation 2.5 to the important case where the matter variables are $(i)$ the baryonic density of the fluid $\rho_{*}=\sqrt{-g} \rho u^{0}$ (with $g=\operatorname{det} g_{\mu \nu}$ ), $(i i)$ the fluid coordinate 3-velocity field $v^{i}=c u^{i} / u^{0}$, and (iii) the entropy per unit mass $s$, i.e. $g_{\mu \nu}=g_{\mu \nu}\left(x^{\alpha}, \rho_{*}, v^{p}, s\right)$.

By using the transformation properties of $\rho$ (scalar), $u^{\mu}$ (vector) and $\sqrt{-g}$ (density), we find at the linear order after a straightforward calculation:

$$
\begin{aligned}
\delta^{*} \rho_{*}= & \partial_{i}\left(\rho_{*} \varepsilon_{x^{\prime}}^{i}\right)-\frac{v^{i}}{c} \partial_{i} \varepsilon_{x^{\prime}}^{0} \rho_{*}+\frac{\varepsilon_{x^{\prime}}^{0}}{c} \partial_{t} \rho_{*} \\
= & \partial_{i}\left(\rho_{*} \varepsilon_{x^{\prime}}^{i}-\rho_{*} \frac{v^{i}}{c} \varepsilon_{x^{\prime}}^{0}\right), \\
\delta^{*} v^{i}= & \frac{1}{c} \partial_{t}\left(\varepsilon_{x^{\prime}}^{0} v^{i}\right)+\varepsilon_{x^{\prime}}^{j} \partial_{j} v^{i}-v^{j} \partial_{j} \varepsilon_{x^{\prime}}^{i} \\
& -\partial_{t} \varepsilon_{x^{\prime}}^{i}+\frac{v^{i} v^{j}}{c} \partial_{j} \varepsilon_{x^{\prime}}^{0} \\
= & \frac{d}{d t}\left(-\varepsilon_{x^{\prime}}^{i}+\frac{v^{i}}{c} \varepsilon_{x^{\prime}}^{0}\right)-\left(-\varepsilon_{x^{\prime}}^{j}+\frac{\varepsilon_{x^{\prime}}^{0}}{c} v^{j}\right) \partial_{j} v^{i}, \\
\delta^{*} s= & \frac{\varepsilon_{x^{\prime}}^{0}}{c} \partial_{t} s+\varepsilon_{x^{\prime}}^{i} \partial_{i} s,
\end{aligned}
$$

having made use of the continuity equation to obtain the second expression of $\delta^{*} \rho_{*}$ (see section IV). The variation $\delta^{*} v^{i}$ is put into a rather compact form by resorting to the total derivative $d / d t=\partial_{t}+v^{i} \partial_{i}$. By virtue of equation (2.5), the new components of the metric read 


$$
\begin{array}{r}
g_{\mu \nu}^{\prime}=g_{\mu \nu}-D_{\rho_{*}}\left[g_{\mu \nu}\right] \cdot \delta^{*} \rho_{*}-D_{v^{q}}\left[g_{\mu \nu}\right] \cdot \delta^{*} v^{q}-D_{s}\left[g_{\mu \nu}\right] \cdot \delta^{*} s \\
+\varepsilon_{x^{\prime}}^{\lambda} \partial_{\lambda} g_{\mu \nu}+2 g_{\lambda(\mu} \partial_{\nu)} \varepsilon_{x^{\prime}}^{\lambda} \quad(2.6)
\end{array}
$$

If the source is made of one point particle of mass $m$, then $\rho_{*}(\mathbf{y}, t)=m \delta(\mathbf{y}-\mathbf{z}(t))$, and the metric can be viewed as a functional of $\mathbf{z}$ and $\mathbf{v}$,

$g_{\mu \nu}\left(x^{\alpha}, \mathbf{z}(t), \mathbf{v}(t)\right)=\frac{1}{m} \int d^{3} \mathbf{y} g_{\mu \nu}\left(x^{\alpha}, \mathbf{y}, \mathbf{v}(\mathbf{y}, t)\right) \rho_{*}(\mathbf{y}, t)$.

The contribution of the functional derivatives is

$$
\begin{aligned}
& -m\left(D_{\rho_{*}}\left[g_{\mu \nu}\right] \cdot \delta^{*} \rho_{*}+D_{v^{q}}\left[g_{\mu \nu}\right] \cdot \delta^{*} v^{q}\right) \\
& =-\int d^{3} \mathbf{y}\left\{g_{\mu \nu}\left(x^{\alpha}, \mathbf{y}, \mathbf{v}(\mathbf{y}, t)\right) \delta^{*} \rho_{*}\right. \\
& \left.\quad+D_{v_{q}}\left[g_{\mu \nu}\left(x^{\alpha}, \mathbf{y}, \mathbf{v}(\mathbf{y}, t)\right)\right] \cdot \delta^{*} v^{q} \rho_{*}\right\} \\
& =\int d^{3} \mathbf{y}\left\{\left(D_{y^{i}}\left[g_{\mu \nu}\left(x^{\alpha}, \mathbf{y}, \mathbf{v}(\mathbf{y}, t)\right)\right]\right.\right. \\
& \left.\left.\quad+\partial_{i} v^{j} D_{v^{j}}\left[g_{\mu \nu}\left(x^{\alpha}, \mathbf{y}, \mathbf{v}(\mathbf{y}, t)\right)\right]\right)\right\} \cdot\left(\rho_{*} \varepsilon_{x^{\prime}}^{i}-\rho_{*} \frac{v^{i}}{c} \varepsilon_{x^{\prime}}^{0}\right) \\
& \left.\quad-\rho_{*} D_{v_{i}}\left[g_{\mu \nu}\left(x^{\alpha}, \mathbf{y}, \mathbf{v}(\mathbf{y}, t)\right)\right]\right\} \cdot\left(\frac{d}{d t}\left(-\varepsilon_{x^{\prime}}^{i}+\frac{v^{i}}{c} \varepsilon_{x^{\prime}}^{0}\right)\right. \\
& \left.\quad-\left(-\varepsilon_{x^{\prime}}^{j}+\frac{\varepsilon_{x^{\prime}}^{0}}{c} v^{j}\right) \partial_{j} v^{i}\right) \\
& \quad+\frac{d}{d t}\left(-\varepsilon_{x^{\prime}}^{i}+\frac{v^{i}}{c} \varepsilon_{x^{\prime}}^{0}\right) \\
& \left.\quad \times D_{v^{i}}\left[g_{\mu \nu}\left(x^{\alpha}, \mathbf{y}, \mathbf{v}(\mathbf{y}, t)\right)\right]\right\} \delta(\mathbf{y}-\mathbf{z}(t)),
\end{aligned}
$$

which shows that

$$
\begin{aligned}
D_{\rho_{*}}\left[g_{\mu \nu}\right] \cdot \delta^{*} \rho_{*} & +D_{v^{q}}\left[g_{\mu \nu}\right] \cdot \delta^{*} v^{q} \\
& =D_{z^{i}}\left[g_{\mu \nu}\right] \cdot \delta^{*} z^{i}+D_{v^{i}}\left[g_{\mu \nu}\right] \cdot \frac{d}{d t} \delta^{*} z^{i},
\end{aligned}
$$

where $\delta^{*} z^{i}=-\varepsilon_{x^{\prime}}^{i}(\mathbf{z}(t), t)+v^{i}(t) \varepsilon_{x^{\prime}}^{0}(\mathbf{z}(t), t) / c$. This is in perfect agreement with the linearized boost transformation formulas [3]: $c t^{\prime}=c t-\varepsilon_{x^{\prime}}^{0}(\mathbf{z}(t), t) ; z^{\prime i}\left(t^{\prime}\right)=$ $z^{i}(t)-\varepsilon_{x^{\prime}}^{i}(\mathbf{z}(t), t)$.

\section{ELIMINATION OF HIGHEST TIME DERIVATIVES IN THE METRIC OF A GENERAL PERFECT FLUID}

An analytic expression for that part of the metric that contributes to the $3.5 \mathrm{PN}$ reaction force arising in an isolated system due to gravitational wave emission is available in a Burke-Thorne-like gauge which also fulfills the harmonicity conditions at 1 PN order [1], for general smooth, spatially compact distributions of matter. However, it is not suited for numerical calculations for it depends on seventh order time derivatives of certain multipole moments. A possible cure consists in performing a change of coordinates such that terms of $\delta_{\varepsilon_{x^{\prime}}}^{*} g_{\mu \nu}$ cancel the highest time derivatives in the relevant part of $g_{\mu \nu}$ (the functional dependence will not be specified anymore henceforth). In order to be able to apply the formula (2.5), we first have to make choice of the fields $X_{A}$. It is convenient to take the mass density $\sigma=\left(T^{00}+T^{i i}\right) / c^{2}$, the current density $\sigma_{i}=T^{0 i} / c$, and the stress $\sigma_{i j}=T^{i j}$, following paper [1]. All three quantities have compact spatial support and are Newtonian at the leading order for weakly stressed systems. Moreover, they lead to a simple expression for the metric, based on a parametrization by means of certain potentials of which they are the sources. At the $1 \mathrm{PN}$ order, these are the Poisson integrals:

$$
\begin{aligned}
U(\mathbf{x}, t) & =G \int \frac{d^{3} \mathbf{y}}{|\mathbf{x}-\mathbf{y}|} \sigma(\mathbf{y}, t), \\
U_{i}(\mathbf{x}, t) & =G \int \frac{d^{3} \mathbf{y}}{|\mathbf{x}-\mathbf{y}|} \sigma_{i}(\mathbf{y}, t),
\end{aligned}
$$

as well as the super potential $\chi(\mathbf{x}, t)=G \int d^{3} \mathbf{y} \mid \mathbf{x}-$ $\mathbf{y} \mid \sigma(\mathbf{y}, t)$. They represent the Poisson solutions of the equations $\Delta U \equiv \partial_{i} \partial_{i} U=-4 \pi G \sigma, \Delta U_{i}=-4 \pi G \sigma_{i}$, $\Delta \chi=2 U$ respectively. We shall write $U=\Delta^{-1}(-4 \pi G \sigma)$ or $\chi=\Delta^{-2}(-8 \pi G \sigma)$. It is worth noticing that $U$ reduces to the Newtonian potential when $c$ goes to infinity. The terms of the metric that contribute to the $1 / c^{5}$ and $1 / c^{7}$ part of the equations of motion take also a particularly simple form. They indeed depend on only two "reaction" potentials $U^{\text {reac }}$ and $U_{i}^{\text {reac }}$ involving the mass quadrupole $I_{i j}(t)$, the mass octupole $I_{i j k}(t)$, and the current quadrupole $J_{i j}(t)$ as defined in the Blanchet-Damour formalism (see [14] and references therein):

$$
\begin{aligned}
U^{\mathrm{reac}}(\mathbf{x}, t)= & -\frac{G}{5 c^{5}} x^{i j} I_{i j}^{(5)}(t)+\frac{G}{c^{7}}\left[\frac{1}{189} x^{i j k} I_{i j k}^{(7)}(t)\right. \\
& \left.-\frac{1}{70} x^{k k} x^{i j} I_{i j}^{(7)}(t)\right]+\mathcal{O}\left(\frac{1}{c^{8}}\right), \\
U_{i}^{\mathrm{reac}}(\mathbf{x}, t)= & \frac{G}{c^{5}}\left[\frac{1}{21} \hat{x}^{i j k} I_{j k}^{(6)}(t)\right. \\
& \left.-\frac{4}{45} \epsilon_{i j k} x^{j m} J_{k m}^{(5)}(t)\right]+\mathcal{O}\left(\frac{1}{c^{6}}\right),
\end{aligned}
$$

the symbol $\hat{x}^{i j k \cdots}$, or equivalently $x^{<i j k \ldots>}$, being a short form for the symmetric trace-free (STF) part of $x^{i j k \ldots} \equiv$ $x^{i} x^{j} x^{k} \ldots$, and $I_{i j}^{(n)}(t)$, an alternative notation for the $n$th time derivative $d^{n} I_{i j}(t) / d t^{n}$. These potentials differ from $V^{\text {reac }}$ and $V_{i}^{\text {reac }}$ used in 14 respectively by terms of order $1 / c^{8}$ and $1 / c^{6}$ which do not play any role at our level [1]. We give for completeness the multipole moments entering $U^{\text {reac }}$ and $U_{i}^{\text {reac }}$ as functionals of $\sigma$ and $\sigma_{i}$ :

$$
\begin{aligned}
I_{i j} & =\int d^{3} \mathbf{y}\left(\hat{y}^{i j} \sigma+\frac{1}{14 c^{2}} y^{k k} \hat{y}^{i j} \partial_{t}^{2} \sigma-\frac{20}{21 c^{2}} \hat{y}^{i j k} \partial_{t} \sigma_{k}\right), \\
I_{i j k} & =\int d^{3} \mathbf{y} \hat{y}^{i j k} \sigma+\mathcal{O}\left(\frac{1}{c^{2}}\right),
\end{aligned}
$$




$$
J_{i j}=\int d^{3} \mathbf{y} \epsilon_{k m<i} \hat{y}^{j>k} \sigma_{m},
$$

with $\epsilon_{k m i}$ holding for the Euclidean Levi-Civita tensor (in three dimensions). Having the potentials $U, U_{i}, \chi$, $U^{\text {reac }}$, and $U_{i}^{\text {reac }}$ to our disposal, we can write the metric components as

$$
\begin{aligned}
g_{00}= & -1+\frac{2}{c^{2}}\left(U+U^{\text {reac }}\right)+\frac{1}{c^{4}}\left[\partial_{t}^{2} \chi-2 U^{2}-4 U U^{\text {reac }}\right] \\
& +\left(g_{00}\right)_{(6+8)}+\mathcal{O}\left(\frac{1}{c^{10}}\right), \\
g_{0 i}= & -\frac{4}{c^{3}}\left(U_{i}+U_{i}^{\text {reac }}\right)+\left(g_{0 i}\right)_{(5+7)}+\mathcal{O}\left(\frac{1}{c^{9}}\right), \quad(3.4 \mathrm{~b}) \\
g_{i j}= & \delta_{i j}\left[1+\frac{2}{c^{2}}\left(U+U^{\text {reac }}\right)\right]+\left(g_{i j}\right)_{(4+6)}+\mathcal{O}\left(\frac{1}{c^{8}}\right),
\end{aligned}
$$

where the indices between brackets indicate the order of the term they refer to, so that $g_{00(6+8)}$ denotes the full set of $1 / c^{6}$ and $1 / c^{8}$ terms entering $g_{00}$.

It should be stressed that the $1 / c^{2}$ order in the corrections to the flat metric entering the right-hand side of equation (3.4) is not the post-Newtonian order, the latter being rather defined as the level of contributions to the equations of motion. Since $\left(g_{00}\right)_{(2)}$ is responsible for the Newtonian force, the terms $\left(g_{00}\right)_{(4)},\left(g_{0 i}\right)_{(3)}$, and $\left(g_{i j}\right)_{(2)}$ that come just after in the power expansion are evidently post-Newtonian. When the matter variables $X_{A}$ are chosen in a way they have a non-zero Newtonian limit $X_{A(0)}=X_{A}+\mathcal{O}\left(1 / c^{2}\right)$ and derive from the trajectory of the fluid in the configuration space independently from the metric, the $1 / c^{5}$ and $1 / c^{7}$ terms of the equations of motion identify with the reaction force in the post-Newtonian scheme. Other set of variables of the type $X_{A}=X_{A(0)}+\mathcal{O}\left(1 / c^{2}\right)$ with $X_{A(0)} \neq 0$ may be equivalently used provided all expansions are performed consistently including the Newtonian, 1 PN, 2.5 PN, and 3.5 PN terms. The coupling between space-time curvature and radiation being a $4 \mathrm{PN}$ effect, the even orders are purely conservative. It is thus convenient to split $g_{\mu \nu}$ into an odd $\left(g_{\mu \nu}\right)_{\text {odd }}$ and an even part $\left(g_{\mu \nu}\right)_{\text {even }}$, named after the parity of the terms they generate in the equations of motion expressed with the help of the variables that have been adopted. As an example, $\left(g_{00}\right)_{(7)},\left(g_{0 i}\right)_{(6)}$ or $\left(g_{i j}\right)_{(5)}$ will belong to the odd part, while $\left(g_{00}\right)_{(2)},\left(g_{0 i}\right)_{(3)}$ or $\left(g_{i j}\right)_{(2)}$ will enter the even one. In our problem, the odd (resp. even) part includes the 2.5 and 3.5 PN (resp. Newtonian and post-Newtonian) corrections. The even part corrections that are beyond the $1 \mathrm{PN}$ approximation do not play any role and can be let unspecified.

We propose now to conduct a coordinate transformation intended to reduce, as far as possible, the order of time derivatives appearing in the original metric components. This can be achieved by eliminating the potentials $U_{(7)}^{\text {reac }}$ and $U_{i(5)}^{\text {reac }}$ containing the highest orders of derivation. In addition, we require that the new gauge identifies with the standard post-Newtonian one, $\partial_{i} g_{0 i}^{\prime}-\frac{1}{2} \partial_{0} g_{i i}^{\prime}=\mathcal{O}\left(1 / c^{5}\right), \partial_{j} g_{i j}^{\prime}-\frac{1}{2} \partial_{i}\left(g_{j j}^{\prime}-g_{00}^{\prime}\right)=\mathcal{O}\left(1 / c^{4}\right)$, which is itself equivalent to the Arnowitt-Deser-Misner (ADM) gauge 15] up to the $1 \mathrm{PN}$ order. As the original 1 PN gravitational field (3.4) satisfies the harmonicity conditions in the near zone (i.e. the region of space including the system in which the post-Newtonian approximation is valid) modulo $1.5 \mathrm{PN}$ corrections, the functions $\varepsilon_{x^{\prime}}^{\mu}$ corresponds at the leading order to the difference between harmonic and ADM coordinates. After paper [3], we have $\varepsilon_{x^{\prime}}^{0}=\mathcal{O}\left(1 / c^{3}\right)$ and $\varepsilon_{x^{\prime}}^{i}=\mathcal{O}\left(1 / c^{4}\right)$, which shows that non-linear terms of the kind $\varepsilon_{x^{\prime}}^{\lambda_{1} \lambda_{2} \ldots} \partial_{\lambda_{1} \lambda_{2} \ldots} D \ldots\left[g_{\mu \nu}\right] \ldots=$ $\mathcal{O}\left(1 / c^{10}\right)$ drop out in equation (2.3). The only nonlinearities that may remain are those generated by the functional variations $\delta_{\varepsilon_{x^{\prime}}}^{*} \sigma, \delta_{\varepsilon_{x^{\prime}}}^{*} \sigma_{i}$, and $\delta_{\varepsilon_{x^{\prime}}}^{*} \sigma_{i j}$. To know whether they can be neglected or not, we have to determine the post-Newtonian order of the three latter quantities. The linear approximation is certainly sufficient for this purpose. We compute $\sigma\left(y^{\prime \alpha}, g_{\alpha \beta}^{\prime}\left(z^{\gamma}, X_{B}^{\prime}\right)\right)$, $\sigma_{i}\left(y^{\prime \alpha}, g_{\alpha \beta}^{\prime}\left(z^{\gamma}, X_{B}^{\prime}\right)\right)$, and $\sigma_{i j}\left(y^{\prime \alpha}, g_{\alpha \beta}^{\prime}\left(z^{\gamma}, X_{B}^{\prime}\right)\right)$, using the gauge transformation law $T^{\mu \nu}\left(y^{\prime \alpha}, g_{\alpha \beta}^{\prime}\left(z^{\gamma}, X_{B}^{\prime}\right)\right)=$ $T^{\mu \nu}\left(y^{\alpha}, g_{\alpha \beta}\left(z^{\gamma}, X_{B}\right)\right)-2 \partial_{\lambda} \varepsilon_{x^{\prime}}^{(\mu} T^{\nu) \lambda}\left(y^{\alpha}, g_{\alpha \beta}\left(z^{\gamma}, X_{B}\right)\right)+$ $\mathcal{O}\left(\varepsilon^{2}\right)$ for the stress-energy tensor. After straightforward calculations, we get

$$
\begin{aligned}
\delta_{\varepsilon_{x^{\prime}}}^{*} \sigma= & \varepsilon_{x^{\prime}}^{k} \partial_{k} \sigma-\frac{2}{c} \sigma \partial_{t} \varepsilon_{x^{\prime}}^{0}+\frac{1}{c} \varepsilon_{x^{\prime}}^{0} \partial_{t} \sigma-\frac{2}{c} \sigma_{i} \partial_{i} \varepsilon_{x^{\prime}}^{0} \\
& -\frac{2}{c^{2}} \sigma_{i} \partial_{t} \varepsilon_{x^{\prime}}^{i}-\frac{2}{c^{2}} \sigma_{i k} \partial_{k} \varepsilon_{x^{\prime}}^{i} \\
& +\frac{2}{c^{3}} \sigma_{k k} \partial_{t} \varepsilon_{x^{\prime}}^{0}+\mathcal{O}\left(\varepsilon^{2}\right)+\mathcal{O}\left(\left(\delta_{\varepsilon_{x^{\prime}}}^{*} g\right)^{2}\right), \\
\delta_{\varepsilon_{x^{\prime}}}^{*} \sigma_{i}= & -\sigma \partial_{t} \varepsilon_{x^{\prime}}^{i}-\sigma_{j} \partial_{j} \varepsilon_{x^{\prime}}^{i}+\varepsilon_{x^{\prime}}^{j} \partial_{j} \sigma_{i}-\frac{1}{c} \sigma_{i} \partial_{t} \varepsilon_{x^{\prime}}^{0} \\
& +\frac{1}{c} \varepsilon_{x^{\prime}}^{0} \partial_{t} \sigma_{i}-\frac{1}{c} \sigma_{i j} \partial_{j} \varepsilon_{x^{\prime}}^{0} \\
& +\frac{1}{c^{2}} \sigma_{j j} \partial_{t} \varepsilon_{x^{\prime}}^{i}+\mathcal{O}\left(\varepsilon^{2}\right)+\mathcal{O}\left(\left(\delta_{\varepsilon_{x^{\prime}}}^{*} g\right)^{2}\right), \\
\delta_{\varepsilon_{x^{\prime}}}^{*} \sigma_{i j}= & -2 \sigma_{(i} \partial_{t} \varepsilon_{x^{\prime}}^{j)}-2 \partial_{k} \varepsilon_{x^{\prime}}^{(i} \sigma_{j) k}+\varepsilon_{x^{\prime}}^{k} \partial_{k} \sigma_{i j} \\
& +\frac{1}{c} \varepsilon_{x^{\prime}}^{0} \partial_{t} \sigma_{i j}+\mathcal{O}\left(\varepsilon^{2}\right)+\mathcal{O}\left(\left(\delta_{\varepsilon_{x^{\prime}}}^{*} g\right)^{2}\right),
\end{aligned}
$$

which means notably that $\delta_{\varepsilon_{x^{\prime}}}^{*} X_{A}=\mathcal{O}\left(1 / c^{4}\right)=$ $\mathcal{O}\left(\varepsilon_{x^{\prime}}^{i}, \varepsilon_{x^{\prime}}^{0} / c\right)$. Consequently, there cannot be nonlinear contributions in $\delta_{\varepsilon_{x^{\prime}}}^{*} g_{\mu \nu}$, and equation (2.5) yields

$$
\begin{aligned}
\left(\delta_{\varepsilon_{x^{\prime}}}^{*} g_{00}\right)_{\text {odd }}= & -\frac{2}{c} \partial_{t} \varepsilon_{x^{\prime}(6+8)}^{0}+\frac{4}{c^{3}} U \partial_{t} \varepsilon_{x^{\prime}(6)}^{0} \\
& +\frac{2}{c}\left(g_{0 i}\right)_{(3)} \partial_{t} \varepsilon_{x^{\prime}(5)}^{i}+\frac{2}{c^{3}} \varepsilon_{x^{\prime}(6)}^{0} \partial_{t} U \\
& +\varepsilon_{x^{\prime}(5)}^{i} \partial_{i}\left(g_{00}\right)_{(4)}+\frac{2}{c^{2}} \varepsilon_{x^{\prime}(5+7)}^{i} \partial_{i} U \\
& -\frac{2}{c^{2}} \int d^{3} \mathbf{y} \frac{\delta U}{\delta X_{A}}\left(\delta_{\varepsilon_{x^{\prime}}}^{*} X_{A}\right)_{(5+7)} \\
& -\int d^{3} \mathbf{y} \frac{\delta\left(g_{00}\right)_{(4)}}{\delta X_{A}}\left(\delta_{\varepsilon_{x^{\prime}}}^{*} X_{A}\right)_{(5)}, \\
\left(\delta_{\varepsilon_{x^{\prime}}}^{*} g_{0 i}\right)_{\text {odd }}= & -\partial_{i} \varepsilon_{x^{\prime}(6+8)}^{0}+\frac{1}{c} \partial_{t} \varepsilon_{x^{\prime}(5+7)}^{i}
\end{aligned}
$$




$$
\begin{aligned}
& +\frac{2}{c^{2}} U \partial_{i} \varepsilon_{x^{\prime}(6)}^{0}+\frac{2}{c^{3}} U \partial_{t} \varepsilon_{x^{\prime}(5)}^{i} \\
& +\left(g_{0 j}\right)_{(3)} \partial_{i} \varepsilon_{x^{\prime}(5)}^{j}+\varepsilon_{x^{\prime}(5)}^{j} \partial_{j}\left(g_{(0 i)}\right)_{(3)} \\
& -\int d^{3} \mathbf{y} \frac{\delta\left(g_{0 i}\right)_{(3)}}{\delta X_{A}}\left(\delta_{\varepsilon_{x^{\prime}}}^{*} X_{A}\right)_{(5)} \\
\left(\delta_{\varepsilon_{x^{\prime}}}^{*} g_{i j}\right)_{\text {odd }}= & 2 \partial_{(i} \varepsilon_{x^{\prime}(5+7)}^{j)}+\frac{4}{c^{2}} U \partial_{(i} \varepsilon_{x^{\prime}(5)}^{j)} \\
& +\frac{2}{c^{2}} \varepsilon_{x^{\prime}(5)}^{k} \partial_{k} U \delta^{i j} \\
& -\frac{2}{c^{2}} \delta^{i j} \int d^{3} \mathbf{y} \frac{\delta U}{\delta X_{A}}\left(\delta_{\varepsilon_{x^{\prime}}}^{*} X_{A}\right)_{(5)}
\end{aligned}
$$

On the other hand, relations (3.3) and (3.4) tell us that the time derivatives of highest order in $g_{\mu \nu}^{\prime}$, namely $\partial_{t}^{6}$ and $\partial_{t}^{7}$, come $(i)$ from the first correction $U_{(7)}^{\text {reac }}$ of the reaction potential $U^{\text {reac }}$ that parametrizes the $3.5 \mathrm{PN}$ part of $g_{00}^{\prime}$, and (ii) from the leading approximation of the reaction potential that contributes to the $3.5 \mathrm{PN}$ part of $g_{0 i}^{\prime}, U_{i}^{\text {reac }}=U_{i(5)}^{\text {reac }}$. We shall restrict ourselves to the case where the new metric can be identified with a function of certain elementary potentials $P_{1}, P_{2}, \ldots$ These potentials are supposed to be spatial integrals whose sources are $a$ priori products of ( $i$ ) densities such as $\sigma, \sigma_{i}, \sigma_{i j}$, or their derivatives evaluated at the location of the integration variable $\mathbf{y}$ at time $t,(i i)$ some factors depending on the field point $\mathbf{x}$, and possibly (iii) instantaneous potentials of the same kind. Typical examples are $U, U_{i}$, or $U_{(5)}^{\text {reac }}$. That the latter quantity is indeed of the required type, this can be seen by letting the factor $x^{i j}$ and the time derivatives go under the integration symbol in the first term of relation (3.2a). Following this hypothesis, we shall search for $\varepsilon_{x^{\prime}}^{\mu}$ as a function of $U, U_{i}$, or some other potentials $P_{1}, P_{2} \ldots$ Moreover, our choice must be such that at least $U_{(7)}^{\text {reac }}$ simplifies with terms coming from the gauge transformation (3.6).

To be more explicit, we come back to equations (3.6a). Together with equations (3.4), they imply

$$
\begin{aligned}
\left(g_{00}^{\prime}\right)_{(7+9)}= & \left(h_{00}\right)_{(9)}-\frac{2}{c} \partial_{t} \varepsilon_{x^{\prime}(6+8)}^{0}+\frac{2}{c^{2}} \varepsilon_{x^{\prime}(5+7)}^{i} \partial_{i} U \\
& -\frac{2}{c^{2}} \int d^{3} \mathbf{y} \frac{\delta U}{\delta \sigma}\left(\delta_{\varepsilon_{x^{\prime}}}^{*} \sigma\right)_{(5+7)}+\frac{2}{c^{2}} U_{(5+7)}^{\mathrm{reac}}, \\
\left(g_{0 i}^{\prime}\right)_{(6+8)}= & \left(h_{0 i}\right)_{(8)}-\partial_{i} \varepsilon_{x^{\prime}(6+8)}^{0} \\
& +\frac{1}{c} \partial_{t} \varepsilon_{x^{\prime}(5+7)}^{i}-\frac{4}{c^{3}} U_{i(5)}^{\mathrm{reac}}, \\
\left(g_{i j}^{\prime}\right)_{(5+7)}= & \left(h_{i j}\right)_{(7)}+2 \partial_{(i} \varepsilon_{x^{\prime}(5+7)}^{j)}+\frac{2}{c^{2}} \delta^{i j} U_{(5)}^{\mathrm{reac}} .
\end{aligned}
$$

For simplicity, the $3.5 \mathrm{PN}$ terms that are functionals of $\varepsilon_{x^{\prime}(6)}^{0}\left(P_{1}, P_{2}, \ldots\right)$ or $\varepsilon_{x^{\prime}(5)}^{i}\left(P_{1}, P_{2}, \ldots\right)$ and functions of potentials involving second order time derivatives, at most, were gathered in the matrix $h_{\mu \nu}$. At the leading order, equation (3.7a) reduces to

$$
\begin{aligned}
\left(g_{00}^{\prime}\right)_{(7)}=-\frac{2}{c} \partial_{t} \varepsilon_{x^{\prime}(6)}^{0}+\frac{2}{c^{2}} \varepsilon_{x^{\prime}(5)}^{i} \partial_{i} U & \\
& -\frac{2}{c^{2}} \int d^{3} \mathbf{y} \frac{\delta U}{\delta \sigma}\left(\delta_{\varepsilon_{x^{\prime}}}^{*} \sigma\right)_{(5)}+\frac{2}{c^{2}} U_{(5)}^{\mathrm{reac}}
\end{aligned}
$$

As long as $U_{(5)}^{\text {reac }}$ appears explicitly in the gravitational field, we shall have to evaluate the fifth order time derivative $I_{i j}^{(5)}(t)$ in numerical simulations. We can avoid this by imposing that the reaction potential $U_{(5)}^{\text {reac }}$ cancels the contribution to $\left(g_{00}^{\prime}\right)_{(7)}$ that is linear in $\varepsilon_{x^{\prime}(6)}^{0}$ or $\varepsilon_{x^{\prime}(5)}^{i}$, which amounts to demanding that $\varepsilon_{x^{\prime}(6)}^{0}\left(\right.$ resp. $\left.\varepsilon_{x^{\prime}(5)}^{i}\right)$ decomposes into terms containing time derivatives of fourth order at most, plus terms $\varepsilon_{x^{\prime}(6)}^{0 \text { (part) }}$ (resp. $\varepsilon_{x^{\prime}(5)}^{i \text { (part I) }}$ or

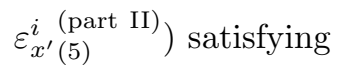

$$
\begin{aligned}
& -\frac{2}{c} \partial_{t} \varepsilon_{x^{\prime}(6)}^{0(\text { part })}+\frac{2}{c^{2}} \varepsilon_{x^{\prime}(5)}^{i(\text { part I) }} \partial_{i} U \\
& \quad-\frac{2}{c^{2}} \int d^{3} \mathbf{y} \frac{\delta U}{\delta \sigma} \varepsilon_{x^{\prime}(5)}^{k(\text { part II) }} \partial_{k} \sigma+\frac{2}{c^{2}} U_{(5)}^{\text {reac }}=0
\end{aligned}
$$

While either $2 \varepsilon_{x^{\prime}(5)}^{i(\text { part I) }} \partial_{i} U / c^{2}$ or $-2 \int d^{3} \mathbf{y} \quad(\delta U / \delta \sigma)$ $\varepsilon_{x^{\prime}(5)}^{k \text { (part II) }} \partial_{k} \sigma / c^{2}$ contribute to the cancellation of $2 U_{5}^{\text {reac }} / c^{2}$, the quantities $\varepsilon_{x^{\prime}(5)}^{i \text { part(I) }}$ or $\varepsilon_{x^{\prime}(5)}^{i \text { part(II) }}$ are, by contrast, explicit functions of $U_{(5)}^{\mathrm{reac}}$. If $\varepsilon_{x^{\prime}(5)}^{i \text { part(I) }} \neq 0$ or $\varepsilon_{x^{\prime}(5)}^{i \text { part(II) }} \neq 0$, the fifth derivative $I_{i j}^{(5)}(t)$ still occurs in the metric by virtue of the relation $g_{i j}^{\prime}=2 \partial_{(i} \varepsilon_{x^{\prime}(5)}^{j)}+$ $\mathcal{O}\left(1 / c^{7}\right)$. This eventuality being rejected, $2 U_{(5)}^{\text {reac }} / c^{2}$ must simplify with $-2 \partial_{t} \varepsilon_{x^{\prime}(6)}^{0 \text { (part) }} / c$ exclusively, i.e.

$$
-\frac{2}{c} \partial_{t} \varepsilon_{x^{\prime}(6)}^{0(\text { part })}+\frac{2}{c^{2}} U_{(5)}^{\text {reac }}=0 .
$$

Therefore, we may construct $\varepsilon_{x^{\prime}(6)}^{0}$ as the sum of $U_{(5)}^{\mathrm{reac}(-1)} / c=-G x^{i j} I_{i j}^{(4)}(t) /\left(5 c^{6}\right)$ (antiderivative of $U_{(5)}^{\text {reac }} / c$ that vanishes when $I_{i j}(t)=0$ ), plus some arbitrary function $\varepsilon_{x^{\prime}(6)}^{0(\mathrm{arb})}$ that does not involve derivatives of order higher than four:

$$
\varepsilon_{x^{\prime}(6)}^{0}=\frac{1}{c} U_{(5)}^{\mathrm{reac}(-1)}+\varepsilon_{x^{\prime}(6)}^{0(\mathrm{arb})} .
$$

As a matter of fact, the choice of $\varepsilon_{x^{\prime}(6)}^{0 \text { (arb) }}$ together with that of $\varepsilon_{x^{\prime}(5)}^{i}$ entirely determines the maximal order $n_{d}\left(\left(g_{00}^{\prime}\right)_{(7)}\right)$ of time derivations in $\left(g_{00}^{\prime}\right)_{(7)}$. Next, the expression for the odd $0 i$ components of the new metric (3.7b) at order $2.5 \mathrm{PN}$,

$$
\left(g_{0 i}^{\prime}\right)_{(6)}=-\partial_{i} \varepsilon_{x^{\prime}(6)}^{0}+\frac{1}{c} \partial_{t} \varepsilon_{x^{\prime}(5)}^{i},
$$

shows that $n_{d}\left(\left(g_{0 i}^{\prime}\right)_{(6)}\right)=n_{d}\left(\partial_{i} \varepsilon_{x^{\prime}(6)}^{0}\right)=4$, unless $-\partial_{i} \varepsilon_{x^{\prime}(6)}^{0 \text { (part) }}$ cancels one of the terms entering $\partial_{t} \varepsilon_{x^{\prime}(5)}^{i} / c$. 
In the latter case, $\left(g_{0 i}^{\prime}\right)_{(6)}$ reduces to $-\partial_{i} \varepsilon_{x^{\prime}(6)}^{0(\mathrm{arb})}+$ $\partial_{t} \varepsilon_{x^{\prime}(5)}^{i(\mathrm{arb})} / c$, where $\varepsilon_{x^{\prime}(5)}^{i(\mathrm{arb})}$ is an arbitrary function of elementary potentials. We have then

$$
n_{d}\left(\left(g_{0 i}^{\prime}\right)_{(6)}\right)=\max \left[n_{d}\left(\varepsilon_{x^{\prime}(6)}^{0(\operatorname{arb})}\right), 1+n_{d}\left(\varepsilon_{x^{\prime}(5)}^{i(\operatorname{arb})}\right)\right] .
$$

Since $n_{d}\left(\left(g_{0 i}^{\prime}\right)_{(6)}\right)$ as well as $n_{d}\left(\left(g_{00}^{\prime}\right)_{(7)}\right)$ are adjustable, we shall succeed in further lowering the order of temporal derivatives if $n_{d}\left(\left(g_{i j}^{\prime}\right)_{(5)}\right) \leq 3$. To know whether this inequality is fulfilled, we solve the equation $-\partial_{i} \varepsilon_{x^{\prime}(6)}^{0 \text { (part) }}+$ $\partial_{t} \varepsilon_{x^{\prime}(5)}^{i \text { (part) }} / c=0$ for $\varepsilon_{x^{\prime}(5)}^{i \text { (part) }} \equiv \varepsilon_{x^{\prime}(5)}^{i}-\varepsilon_{x^{\prime}(5)}^{i \text { (arb) }}$. The solution that vanishes when $I_{i j}(t)=0$ reads

$$
\varepsilon_{x^{\prime}(5)}^{i(\text { part })}=c \partial_{i} \varepsilon_{x^{\prime}(6)}^{0(\operatorname{part})(-1)}=\partial_{i} U_{(5)}^{\mathrm{reac}(-2)},
$$

which leads to odd metric space components of the form $\left(g_{i j}^{\prime}\right)_{(5)}=\partial_{(i} \varepsilon_{x^{\prime}(5)}^{j)}-2 G I_{i j}^{(3)}(t) /\left(5 c^{5}\right)$, with $n_{d}\left(\left(g_{i j}^{\prime}\right)_{(5)}\right)=3$. No function of $\varepsilon_{x^{\prime}(6)}^{0(\operatorname{arb})}$ or $\varepsilon_{x^{\prime}(5)}^{i(\operatorname{arb})}$ having a maximal time derivative order smaller than 3 are capable to rule out the second term, so that we may take $\varepsilon_{x^{\prime}(6)}^{0(\mathrm{arb})}=\varepsilon_{x^{\prime}(5)}^{i(\mathrm{arb})}=0$.

Having specified the gauge vector at the $2.5 \mathrm{PN}$ order, we can proceed to the next approximation, and determine both $\varepsilon_{x^{\prime}(8)}^{0}$ and $\varepsilon_{x^{\prime}(7)}^{i}$ by using the same method as employed above. The matrix $h_{\mu \nu}$, depending on none of the unknown functions, is entirely fixed at this stage. The highest order temporal derivations again arise in the reaction potential. At this level, we have $n_{d}\left(\left(g_{\mu \nu}^{\prime}\right)_{\text {odd }}\right)=n_{d}\left(U_{(7)}^{\text {reac }}\right)=7$. As before, we intend to make this number decrease by discarding the term $U_{(7)}^{\text {reac }}$ from the metric (3.7). Its complete or partial absorption into $\varepsilon_{x^{\prime}(7)}^{i}$ would result in a explicit dependence: $\varepsilon_{x^{\prime}(7)}^{i}=\varepsilon_{x^{\prime}(7)}^{i}\left(U_{(7)}^{\text {reac }}\right)$, which would entail the appearance of time derivatives of seventh order in the spatial part (3.7c) of $g_{\mu \nu}^{\prime}$. Considering that $\left(g_{i j}^{\prime}\right)_{(7)}-2 \partial_{(i} \varepsilon_{x^{\prime}(7)}^{j)}$ contains fifth order derivatives at most, these high order time derivatives could not be erased by means of other contributions. We would end up to the same value of $n_{d}\left(\left(g_{\mu \nu}^{\prime}\right)_{\text {odd }}\right)$ as before: $n_{d}\left(\left(g_{\mu \nu}^{\prime}\right)_{\text {odd }}\right)=n_{d}\left(\left(g_{i j}^{\prime}\right)_{(7)}\right)=7$. Because it is not acceptable, we must rather incorporate $U_{(7)}^{\text {reac }}$ into $\varepsilon_{x^{\prime}(8)}^{0}$, and set

$$
\begin{aligned}
\varepsilon_{x^{\prime}(8)}^{0}=\frac{1}{c} U_{(7)}^{\mathrm{reac}(-1)}+\varepsilon_{x^{\prime}(8)}^{0(\mathrm{arb})}, & \\
& n_{d}\left(\varepsilon_{x^{\prime}(8)}^{0(\mathrm{arb})}\right) \leq 6,
\end{aligned}
$$

$n_{d}\left(\left(g_{\mu \nu}^{\prime}\right)_{\text {odd }}\right)$ going automatically down to 6 . The highest order time derivatives actually come from the terms $-\partial_{i} \varepsilon_{x^{\prime}(8)}^{0}$ and $-4 U_{i(5)}^{\text {reac }} / c^{3}$ in $\left(g_{0 i}^{\prime}\right)_{(8)}$, but they can be eliminated if a part of $-\partial_{i} \varepsilon_{x^{\prime}(8)}^{0(\operatorname{arb})}+\partial_{t} \varepsilon_{x^{\prime}(7)}^{i}$ exactly cancel them. This happens for

$$
\varepsilon_{x^{\prime}(7)}^{i}=\partial_{i} U_{(7)}^{\mathrm{reac}(-2)}+\frac{4}{c^{2}} U_{i(5)}^{\mathrm{reac}(-1)}+\varepsilon_{x^{\prime}(7)}^{i(\operatorname{arb})},
$$

$$
n_{d}\left(\varepsilon_{x^{\prime}(7)}^{i(\operatorname{arb})}\right) \leq 5 .
$$

The space components of the new 3.5 PN metric are obtained by inserting the expression above into the righthand side of equation (3.7C). This yields a maximal order of temporal derivations equal to $n_{d}\left(\varepsilon_{x^{\prime}(7)}^{i}\right)=5$, whatever functions $\varepsilon_{x^{\prime}(8)}^{0(\operatorname{arb})}$ and $\varepsilon_{x^{\prime}(7)}^{i(\operatorname{arb})}$ we have used. We have still the freedom to specify the "arbitrary" part of $\varepsilon_{x^{\prime}}$ as most convenient for us.

In summary, it is possible to reduce the number $n_{d}\left(g_{\mu \nu}^{\prime}\right)$ from 7 to 5 by performing a suitable (linear) gauge transformation. Equations (3.8), (3.9), (3.10), and (3.11) provide a possible choice for the gauge vector:

$$
\begin{aligned}
& \varepsilon_{x^{\prime}(6+8)}^{0}=\frac{1}{c} \partial_{t} U_{(5+7)}^{\mathrm{reac}(-2)}, \\
& \varepsilon_{x^{\prime}(5+7)}^{i}=\partial_{i} U_{(5+7)}^{\mathrm{reac}(-2)}+\frac{4}{c^{2}} U_{i(5)}^{\mathrm{reac}(-1)},
\end{aligned}
$$

with

$$
\begin{aligned}
U^{\mathrm{reac}(-2)} & =-\frac{G}{5 c^{5}} x^{i j} I_{i j}^{(3)}(t)+\frac{G}{c^{7}}\left[\frac{1}{189} x^{i j k} I_{i j k}^{(5)}(t)\right. \\
& \left.-\frac{1}{70} x^{k k} x^{i j} I_{i j}^{(5)}(t)\right]+\mathcal{O}\left(\frac{1}{c^{8}}\right)
\end{aligned}
$$

and

$$
\begin{aligned}
U_{i}^{\mathrm{reac}(-1)}= & \frac{G}{c^{5}}\left[\frac{1}{21} \hat{x}^{i j k} I_{j k}^{(5)}(t)\right. \\
& \left.-\frac{4}{45} \epsilon_{i j k} x^{j m} J_{k m}^{(4)}(t)\right]+\mathcal{O}\left(\frac{1}{c^{6}}\right)
\end{aligned}
$$

modulo the "arbitrary" part. There exist other interesting alternatives. For instance, we could let the original metric unchanged at the $2.5 \mathrm{PN}$ level while keeping the requirement $n_{d}\left(\left(g_{\mu \nu}^{\prime}\right)_{\text {odd }}\right) \leq 5$ since neither $\left(g_{00}\right)_{(7)}$, $\left(g_{0 i}\right)_{(6)}$, or $\left(g_{i j}\right)_{(5)}$ involve time derivatives of higher order. The coordinate system defined by means of formulas (3.12) present the advantage to coincide with the ADM one up to the 2.5 PN approximation, as we shall see in the next section.

\section{EXPRESSION OF THE ODD METRIC AT THE 3.5 PN ORDER}

For computing the reaction force, we must now finalize the form of the gravitational field by fixing the two arbitrary functions $\varepsilon_{x^{\prime}(8)}^{0(\text { arb })}$ and $\varepsilon_{x^{\prime}(7)}^{i(\text { arb })}$. On one hand, the gauge $x^{\prime \mu}=x^{\mu}-\varepsilon_{x^{\prime}}^{\mu}$ is constructed in such a way that $g_{\mu \nu}^{\prime}$ is precisely the ADM metric $\left(g_{\mu \nu}\right)_{\mathrm{ADM}}$ at the $1 \mathrm{PN}$ order: $g_{\mu \nu}^{\prime}=\left(g_{\mu \nu}\right)_{\mathrm{ADM}}$, plus a $2 \mathrm{PN}$ deviation. On the other hand, $\left(g_{00}^{\prime}\right)_{(9)}$ depends on the $1 \mathrm{PN}$ components of the metric (3.4) in the Burke-Thorne-like gauge introduced in paper [1]. Now, the non-spatial terms of $\left(g_{\mu \nu}\right)_{\mathrm{ADM}}$ differ from those of $g_{\mu \nu}$ at this order. We have:

$$
\left(g_{00}\right)_{\mathrm{ADM}(4)}=-\frac{2}{c^{4}} U^{2},
$$




$$
\left(g_{0 i}\right)_{\mathrm{ADM}(3)}=-\frac{1}{c^{3}} A_{i} \equiv-\frac{1}{c^{3}}\left(4 U_{i}+\frac{1}{2} \partial_{i} \partial_{t} \chi\right) .
$$

In order to homogenize the expression of the $3.5 \mathrm{PN}$ metric, we shall choose the "arbitrary" part of $\varepsilon_{x^{\prime}}$ so as to discard the contribution of $\left(g_{00}\right)_{(4)}$ (resp. $\left.\left(g_{0 i}\right)_{(3)}\right)$ and replace it by that of $\left(g_{00}\right)_{\mathrm{ADM}(4)}$ (resp. $\left.\left(g_{0 i}\right)_{\mathrm{ADM}(3)}\right)$ in $\left(\delta^{*} g_{\mu \nu}^{\prime}\right)_{\text {odd }}$. This is achieved by taking

$$
\begin{aligned}
\varepsilon_{x^{\prime}(8)}^{0(\mathrm{arb})}= & \varepsilon_{x^{\prime}(5)}^{i} \partial_{i} \varepsilon_{x^{\prime}(3)}^{0}-D_{X_{B}}\left[\varepsilon_{x^{\prime}(3)}^{0}\right] \cdot\left(\delta_{\varepsilon_{\mathrm{odd}}^{\beta}}^{*} X_{B}\right)_{(5)} \\
= & \varepsilon_{x^{\prime}(6)}^{0} \partial_{0} \varepsilon_{x^{\prime}(3)}^{0}+\varepsilon_{x^{\prime}(5)}^{i} \partial_{i} \varepsilon_{x^{\prime}(3)}^{0} \\
& -D_{X_{B}}\left[\varepsilon_{x^{\prime}(3)}^{0}\right] \cdot \delta_{\varepsilon_{\mathrm{odd}} X_{B}}^{*} X_{B}+\mathcal{O}\left(\frac{1}{c^{10}}\right), \\
\varepsilon_{x^{\prime}(7)}^{i(\mathrm{arb})}=0= & \varepsilon_{x^{\prime}(6)}^{0} \partial_{0} \varepsilon_{x^{\prime}(4)}^{i}+\varepsilon_{x^{\prime}(5)}^{j} \partial_{j} \varepsilon_{x^{\prime}(4)}^{i} \\
& \quad-D_{X_{B}}\left[\varepsilon_{x^{\prime}(4)}^{i}\right] \cdot \delta_{\varepsilon_{\text {odd }}^{\beta} X_{B}}^{*}+\mathcal{O}\left(\frac{1}{c^{9}}\right),
\end{aligned}
$$

with $\epsilon_{\text {odd }}^{\mu} \equiv\left(\varepsilon_{x^{\prime}(6+8)}^{0}, \varepsilon_{x^{\prime}(5+7)}^{i}\right)$. This statement can be checked either directly or by use of formula (2.4) of section III noticing that the final value of $\varepsilon_{x^{\prime}}$, which reads

$$
\varepsilon_{x^{\prime}}^{\mu}=\varepsilon_{\text {even }}^{\mu}+\varepsilon_{\text {odd }}^{\mu}+\varepsilon_{\text {odd }}^{\lambda} \partial_{\lambda} \varepsilon_{\text {even }}^{\mu}-D_{X_{B}}\left[\varepsilon_{\text {even }}^{\mu}\right] \cdot \delta_{\varepsilon_{\text {odd }}^{\beta} X_{B}}^{*}
$$

if we pose $\varepsilon_{\text {even }}^{\mu}=\left(\varepsilon_{x^{\prime}(3)}^{0}, \varepsilon_{x^{\prime}(4)}^{i}\right)$, is exactly the one we would get by applying successively the two coordinate transformations $x^{\prime \prime \mu}=x^{\mu}-\varepsilon_{\text {even }}^{\mu}\left(x^{\prime \prime \alpha}, X_{A}^{\prime \prime}\right)$ and $x^{\prime \mu}=$ $x^{\prime \prime \mu}-\varepsilon_{\text {odd }}^{\mu}\left(x^{\prime \alpha}, X_{A}^{\prime}\right)$.

The resulting metric $g_{\mu \nu}^{\prime}$ is

$$
\begin{aligned}
g_{00}^{\prime}= & -1+\frac{2}{c^{2}} U-\frac{2}{c^{4}} U^{2}-\frac{4}{c^{4}} U U^{\mathrm{reac}}+\frac{4}{c^{3}} U \partial_{t} \varepsilon_{x^{\prime}(6)}^{0} \\
& -\frac{2}{c^{4}} A_{i} \partial_{t} \varepsilon_{x^{\prime}(5)}^{i}+\frac{2}{c^{3}} \varepsilon_{x^{\prime}(6)}^{0} \partial_{t} U+\frac{1}{c^{4}} \varepsilon_{x^{\prime}(5)}^{i} \partial_{i}\left(-2 U^{2}\right) \\
& +\frac{2}{c^{2}} \varepsilon_{x^{\prime}(5+7)}^{i} \partial_{i} U-\frac{2}{c^{2}} \int d^{3} \mathbf{y} \frac{\delta U}{\delta X_{A}}\left(\delta_{\varepsilon_{x^{\prime}}}^{*} X_{A}\right)_{(5+7)} \\
& -\frac{1}{c^{4}} \int d^{3} \mathbf{y} \frac{\delta\left(-2 U^{2}\right)}{\delta X_{A}}\left(\delta_{\varepsilon_{x^{\prime}}}^{*} X_{A}\right)_{(5)}+\frac{1}{c^{6}}(\ldots) \\
& +\frac{1}{c^{8}}(\ldots)+\mathcal{O}\left(\frac{1}{c^{10}}\right) \\
g_{0 i}^{\prime}= & -\frac{1}{c^{3}} A_{i}+\frac{2}{c^{2}} U \partial_{i} \varepsilon_{x^{\prime}(6)}^{0}+\frac{2}{c^{3}} U \partial_{t} \varepsilon_{x^{\prime}(5)}^{i} \\
& -\frac{1}{c^{3}} A_{j} \partial_{i} \varepsilon_{x^{\prime}(5)}^{j}-\frac{1}{c^{3}} \varepsilon_{x^{\prime}(5)}^{j} \partial_{j} A_{i} \\
& +\frac{1}{c^{3}} \int d^{3} \mathbf{y} \frac{\delta A_{i}}{\delta X_{A}}\left(\delta_{\varepsilon_{x^{\prime}}}^{*} X_{A}\right)_{(5)}+\frac{1}{c^{5}}(\ldots) \\
& +\frac{1}{c^{7}}(\ldots)+\mathcal{O}\left(\frac{1}{c^{9}}\right), \\
g_{i j}^{\prime}= & \delta^{i j}\left[1+\frac{2}{c^{2}} U+\frac{2}{c^{2}} U_{(5)}^{\mathrm{reac}}\right]+2 \partial_{(i} \varepsilon_{x^{\prime}(5+7)}^{j)} \\
& +\frac{4}{c^{2}} U \partial_{(i} \varepsilon_{x^{\prime}(5)}^{j)}+\frac{2}{c^{2}} \varepsilon_{x^{\prime}(5)}^{k} \partial_{k} U \delta^{i j} \\
& -\frac{2}{c^{2}} \delta^{i j} \int d^{3} \mathbf{y} \frac{\delta U}{\delta X_{A}}\left(\delta_{\varepsilon_{x^{\prime}}}^{*} X_{A}\right)_{(5)}+\frac{1}{c^{4}}(\ldots)
\end{aligned}
$$

where $\varepsilon_{x^{\prime}(6+8)}^{0}$ and $\varepsilon_{x^{\prime}(5+7)}^{i}$ are defined in equations (3.12). We remind that $\left(g_{0 i}^{\prime}\right)_{(6)}=0$ by construction. We may now calculate the functional derivatives explicitly. Considering for example the potential $U$, its source contains none of the densities $\sigma_{i}$ or $\sigma_{i j}$, and thus, $\int d^{3} \mathbf{y}\left(\delta U / \delta \sigma_{i}\right) \delta^{*} \sigma_{i}=\int d^{3} \mathbf{y}\left(\delta U / \delta \sigma_{i j}\right) \delta^{*} \sigma_{i j}=0$. The term $\int d^{3} \mathbf{y}(\delta U / \delta \sigma) \delta^{*} \sigma$ is deduced from the evaluation of the difference $U^{\prime}-U$ :

$$
\begin{aligned}
U^{\prime}-U & =G \int \frac{d^{3} \mathbf{y}}{|\mathbf{x}-\mathbf{y}|} \sigma^{\prime}(\mathbf{y}, t)-G \int \frac{d^{3} \mathbf{y}}{|\mathbf{x}-\mathbf{y}|} \sigma(\mathbf{y}, t) \\
& =G \int \frac{d^{3} \mathbf{y}}{|\mathbf{x}-\mathbf{y}|} \delta^{*} \sigma(\mathbf{y}, t)+\mathcal{O}\left(\left[\delta^{*} \sigma\right]^{2}\right),
\end{aligned}
$$

hence

$$
\begin{aligned}
& \int d^{3} \mathbf{y} \frac{\delta U}{\delta \sigma}\left(\delta_{\varepsilon_{x^{\prime}}}^{*} \sigma\right)_{(5+7)} \\
& =G \int \frac{d^{3} \mathbf{y}}{|\mathbf{x}-\mathbf{y}|}\left(\delta_{\varepsilon_{x^{\prime}}}^{*} \sigma\right)_{(5+7)} \\
& =G \int \frac{d^{3} \mathbf{y}}{|\mathbf{x}-\mathbf{y}|}\left[\varepsilon_{x^{\prime}(5+7)}^{k} \partial_{k} \sigma-\frac{2}{c} \sigma \partial_{t} \varepsilon_{x^{\prime}(6)}^{0}+\frac{1}{c} \varepsilon_{x^{\prime}(6)}^{0} \partial_{t} \sigma\right. \\
& \left.-\frac{2}{c} \sigma_{i} \partial_{i} \varepsilon_{x^{\prime}(6)}^{0}-\frac{2}{c^{2}} \sigma_{i} \partial_{t} \varepsilon_{x^{\prime}(5)}^{i}-\frac{2}{c^{2}} \sigma_{i k} \partial_{k} \varepsilon_{x^{\prime}(5)}^{i}\right](\mathbf{y}, t) .
\end{aligned}
$$

The other non-zero functional derivatives present in the metric variation (3.6) are computed following the same procedure.

$$
\begin{gathered}
\int d^{3} \mathbf{y} \frac{\delta A_{i}}{\delta \sigma}\left(\delta_{\varepsilon_{x^{\prime}}}^{*} \sigma\right)_{(5)} \\
=4 \int d^{3} \mathbf{y} \frac{\delta U_{i}}{\delta \sigma}\left(\delta_{\varepsilon_{x^{\prime}}}^{*} \sigma\right)_{(5)}+\frac{1}{2} \int d^{3} \mathbf{y} \frac{\delta\left(\partial_{t} \partial_{i} \chi\right)}{\delta \sigma}\left(\delta_{\varepsilon_{x^{\prime}}}^{*} \sigma\right)_{(5)} \\
=\frac{G}{2} \partial_{t} \partial_{i} \int d^{3} \mathbf{y}|\mathbf{x}-\mathbf{y}|\left[\varepsilon_{x^{\prime}(5)}^{k} \partial_{k} \sigma\right](\mathbf{y}, t) \\
\int d^{3} \mathbf{y} \frac{\delta A_{i}}{\delta \sigma_{j}}\left(\delta_{\varepsilon_{x^{\prime}}}^{*} \sigma_{j}\right)_{(5)} \\
=4 G \int \frac{d^{3} \mathbf{y}}{|\mathbf{x}-\mathbf{y}|}\left[-\sigma \partial_{t} \varepsilon_{x^{\prime}(5)}^{i}-\sigma_{j} \partial_{j} \varepsilon_{x^{\prime}(5)}^{i}\right. \\
\left.+\varepsilon_{x^{\prime}(5)}^{j} \partial_{j} \sigma_{i}\right](\mathbf{y}, t) .
\end{gathered}
$$

By inserting formulas (4.4) into equation (4.3), we get an expression for the metric already well adapted to our purpose. Notably, all potentials elementary potentials but $\chi$ and $G \int d^{3} \mathbf{y}|\mathbf{x}-\mathbf{y}|(\delta \chi / \delta \sigma) \delta_{\varepsilon_{x^{\prime}}}^{*} \sigma$ satisfy Poissontype equations. Nonetheless, in order to make easier the comparison with paper [4], we shall present an alternative form where the functional derivatives are re-written as combinations of Poisson-type integrals on the sources $X_{1}=\sigma$ and $X_{2}=\sigma_{i}$ exclusively. It can be obtained from 
an appropriate handling of the integrands in the potentials (4.4). Originally, they are indeed made of sums of elementary pieces of the kind

$$
\begin{aligned}
& |\mathbf{x}-\mathbf{y}|^{2 p-1} \partial X_{A}(\mathbf{y}, t) \partial \varepsilon_{\text {odd }} \\
& \quad \propto|\mathbf{x}-\mathbf{y}|^{2 p-1} y^{i_{1} i_{2} \ldots i_{l}} \partial X_{A}(\mathbf{y}, t) \times \text { function of time, }
\end{aligned}
$$

for $A=1,2$ and $p, l \in \mathbb{N}$. Now, it is straightforward to show by recurrence that

$y^{i_{1} i_{2} \ldots i_{l}}=x^{i_{1} i_{2} \ldots i_{l}}-\left[x^{i_{1} i_{2} \ldots i_{l-1}}\left(x^{i_{l}}-y^{i_{l}}\right)+\right.$ permutations $]$

$$
\begin{aligned}
& +\left[x^{i_{1} i_{2} \ldots i_{l-2}}\left(x^{i_{l-1}}-y^{i_{l-1}}\right)\left(x^{i_{l}}-y^{i_{l}}\right)+\text { permutations }\right] \\
& +\ldots+(-1)^{l}\left(x^{i_{1}}-y^{i_{1}}\right)\left(x^{i_{2}}-y^{i_{2}}\right) \ldots\left(x^{i_{l}}-y^{i_{l}}\right)
\end{aligned}
$$

so that every piece decomposes into a sum of terms such as $x^{j_{1} j_{2} \ldots j_{k}}\left(x^{i_{1}}-y^{i_{1}}\right)\left(x^{i_{2}}-y^{i_{2}}\right) \ldots\left(x^{i_{l}}-y^{i_{l}}\right)|\mathbf{x}-\mathbf{y}|^{2 p-1}$. Each of them are next transformed by means of the identity

$$
\left(x^{i_{1}}-y^{i_{1}}\right) \ldots\left(x^{i_{l}}-y^{i_{l}}\right)|\mathbf{x}-\mathbf{y}|^{a}=\sum_{m=0}^{[l / 2]}\left[\frac{(-1)^{m} \delta^{i_{1} i_{2}} \ldots \delta^{i_{2 m-1} i_{2 m}}}{(a+2 l-2 m) \ldots(a+2)} \partial_{i_{2 m+1} \ldots i_{l}}|\mathbf{x}-\mathbf{y}|^{a+2 l-2 m}+\text { permutations }\right]
$$

valid for $a>-2$, where the latter relation results from the formula

$$
\begin{array}{r}
\partial_{i_{1} i_{2} \ldots i_{l}}|\mathbf{x}-\mathbf{y}|^{a}=\sum_{m=0}^{[l / 2]} a(a-2) \ldots(a-2 l+2 m+2)|\mathbf{x}-\mathbf{y}|^{a-2 l+2 m}\left[\delta^{i_{1} i_{2}} \ldots \delta^{i_{2 m-1} i_{2 m}}\right. \\
\left.\quad \times\left(x^{i_{2 m+1}}-y^{i_{2 m+1}}\right) \ldots\left(x^{i_{l}}-y^{i_{l}}\right)+\text { permutations }\right]
\end{array}
$$

proved by simple recurrence. The square bracket $[l / 2]$ denotes here the integer part of $l / 2$. We thus see that the initial potentials can be split into a sum of terms matching the pattern:

$$
\begin{aligned}
\int d^{3} \mathbf{y} & x^{j_{1} j_{2} \ldots j_{k}} \partial_{i_{1} i_{2} \ldots i_{l}}|\mathbf{x}-\mathbf{y}|^{2 p-1} \partial X_{A}(\mathbf{y}, t) \\
= & x^{j_{1} j_{2} \ldots j_{k}} \partial \partial_{i_{1} i_{2} \ldots i_{l}} \int d^{3} \mathbf{y}|\mathbf{x}-\mathbf{y}|^{2 p-1} X_{A}(\mathbf{y}, t) .
\end{aligned}
$$

Under this form, they only depend on the Poissonlike integrals $P_{q}=G \int d^{3} \mathbf{y}|\mathbf{x}-\mathbf{y}|^{2 q-1} \sigma(\mathbf{y}, t), P_{i q}=$ $G \int d^{3} \mathbf{y}|\mathbf{x}-\mathbf{y}|^{2 q-1} \sigma_{i}(\mathbf{y}, t)$ as well as $P_{i j q}=G \int d^{3} \mathbf{y} \mid \mathbf{x}-$ $\left.\mathbf{y}\right|^{2 q-1} \sigma_{i j}(\mathbf{y}, t)$. We shall need in particular $P_{0}=U, P_{1}=$ $\chi, P_{i 0}=U_{i}$ as before, but also $\chi_{i}=P_{i 1}$ and $U_{i j}=P_{i j 0}$. As an example, let us apply the latter treatment to the simplest functional derivative $G \int d^{3} \mathbf{y} /|\mathbf{x}-\mathbf{y}| \varepsilon_{x^{\prime}(5)}^{k} \partial_{k} \sigma$. We have:

$$
\begin{aligned}
& G \int \frac{d^{3} \mathbf{y}}{|\mathbf{x}-\mathbf{y}|} \varepsilon_{x^{\prime}(5)}^{k} \partial_{k} \sigma \\
& =G \int \frac{d^{3} \mathbf{y}}{|\mathbf{x}-\mathbf{y}|}\left(-\frac{2 G}{5 c^{5}} y^{i} I_{i j}^{(3)}(t)\right) \partial_{j} \sigma \\
& =-\frac{2 G^{2}}{5 c^{5}} I_{i j}^{(3)}(t) \int d^{3} \mathbf{y}\left[-\frac{\left(x^{i}-y^{i}\right)}{|\mathbf{x}-\mathbf{y}|}+\frac{x^{i}}{|\mathbf{x}-\mathbf{y}|}\right] \partial_{j} \sigma \\
& =-\frac{2 G^{2}}{5 c^{5}} I_{i j}^{(3)}(t)\left[-\int d^{3} \mathbf{y} \frac{\partial}{\partial x^{i}}|\mathbf{x}-\mathbf{y}| \frac{\partial}{\partial y_{j}} \sigma\right. \\
& \left.\quad+x^{i} \int \frac{d^{3} \mathbf{y}}{|\mathbf{x}-\mathbf{y}|} \frac{\partial \sigma}{\partial y_{j}}\right] .
\end{aligned}
$$

After integrating by part and observing that $\partial \mid \mathbf{x}-$ $\left.\mathbf{y}\right|^{a} / \partial y^{i}=-\partial|\mathbf{x}-\mathbf{y}|^{a} / \partial x^{i}$ for any real number $a$, we arrive at the equality: $G \int d^{3} \mathbf{y} \varepsilon_{x^{\prime}(5)}^{k} \partial_{k} \sigma /|\mathbf{x}-\mathbf{y}|=$ $-2 G I_{i j}^{(3)}(t)\left[-\partial_{i j} \chi+x^{i} \partial_{j} U\right] /\left(5 c^{5}\right)$. Processing all the elementary potentials in a similar way leads to the important result

$$
\begin{aligned}
\left(g_{00}^{\prime}\right)_{\text {odd }} & =-\frac{4 G}{5 c^{7}} I_{k l}^{(3)} \partial_{k l} \chi \\
+\frac{2 G}{c^{9}}\left(\frac{4}{5} I_{k l}^{(3)}\left(U \partial_{k l} \chi-U_{k l}\right)\right. & \\
+ & \frac{1}{5} I_{k l}^{(4)}\left(8 \partial_{k} \chi_{l}+\frac{1}{3} \partial_{k l} \partial_{t} P_{2}-x^{k} \partial_{l} \partial_{t} \chi\right) \\
+ & \frac{1}{7} I_{k l}^{(5)}\left(-\frac{8}{15} x^{k} x^{l} U-\frac{11}{15} r^{2} \partial_{k l} \chi-\frac{29}{15} x^{k} \partial_{l} \chi\right. \\
& \left.+\frac{4}{5} x^{k} x^{m} \partial_{m l} \chi+\frac{1}{3} \partial_{k l} P_{2}+\frac{1}{9} x^{m} \partial_{k l m} P_{2}\right) \\
+ & \frac{1}{63} I_{k l m}^{(5)}\left(-\frac{1}{3} \partial_{k l m} P_{2}+2 x^{k} \partial_{l m} \chi\right) \\
- & \left.\frac{16}{45} x^{k} \epsilon_{k l m} J_{l n}^{(4)} \partial_{m n} \chi\right) \\
\left(g_{0 i}^{\prime}\right)_{\text {odd }} & =\frac{G}{5 c^{8}}\left(16 I_{i k}^{(3)} U_{k}+I_{k l}^{(3)}\left(8 \partial_{k l} \chi_{i}+\frac{1}{3} \partial_{i k l} \partial_{t} P_{2}\right)\right. \\
\left.\quad-9 I_{i k}^{(4)} \partial_{k} \chi+I_{k l}^{(4)}\left(-x^{k} \partial_{i l} \chi+\frac{1}{3} \partial_{i k l} P_{2}\right)\right) & 4 G \\
\left(g_{i j}^{\prime}\right)_{\text {odd }} & =-\frac{4 G}{5 c^{5}} I_{i j}^{(3)}
\end{aligned}
$$




$$
\begin{aligned}
+\frac{2 G}{c^{7}}( & -\frac{4}{5} I_{i j}^{(3)} U-\frac{2}{5} \delta^{i j} I_{k l}^{(3)} \partial_{k l} \chi-\frac{11}{105} r^{2} I_{i j}^{(5)} \\
& +\frac{2}{63} x^{k} I_{i j k}^{(5)}+\frac{4}{35} x^{k} x^{(i} I_{j) k}^{(5)} \\
& \left.-\frac{4}{105} \delta^{i j} x^{k} x^{l} I_{k l}^{(5)}-\frac{16}{45} x^{k} \epsilon_{k l(i} J_{j) l}^{(4)}\right),
\end{aligned}
$$

with $r^{2}=x^{m} x^{m}$. The leading components of $\left(g_{\mu \nu}^{\prime}\right)_{\text {odd }}$ are identical to those of the $2.5 \mathrm{PN}$ metric in ADM coordinates. With the help of these formulas, we recover the expression proposed by Rezzolla et al. in paper [4] in absence of mass multipole moments, i.e. when $I_{i_{1} i_{2} \ldots i_{l}}=0$. We conclude that our gauge coincides with the ADM one up to the 2.5 PN order, and generalizes that of Rezzolla and collaborators at the next level.

Once the gravitational field has been evaluated in the new coordinate system, we may take advantage of the freedom in the choice of the matter variables. Variables defined by means of the fluid trajectory in the configuration space without any reference to the metric are particularly appropriate to post-Newtonian calculations, since their Poisson brackets are identical to those of Newtonian theory 16]. We first take the baryonic density $\rho_{*}$ representing the number of baryon weighed by their individual mass per coordinate volume $d^{3} \mathbf{x}$. It reduces to the scalar density $\rho$ at the Newtonian order: $\rho_{*}=\sqrt{-g^{\prime}} \rho u^{0}=\rho+\mathcal{O}\left(1 / c^{2}\right)$. The second variable we shall use for describing the fluid is the coordinate velocity $v^{i}$. It does not depend on the metric as being the Eulerian quantity associated to the Lagrangian velocity $d x^{\mu}\left(x_{0}^{\alpha}, t\right) / d t$ of the particle $x^{\mu}\left(x_{0}^{\alpha}, t\right)$ located at point $x_{0}^{i}$ at time $x_{0}^{0} / c$. We have $v^{i} / c=u^{i} / u^{0}$, and therefore, $v^{i} / c=u^{i}+\mathcal{O}\left(1 / c^{2}\right)$. We shall introduce a zeroth component $v^{0}=c$ when necessary, so that we can write $v^{\mu} / c=u^{\mu} / u^{0}$. For macroscopic systems, the set of variables $\left\{\rho_{*}, v^{i}\right\}$ is completed by the entropy $s$.

The relation between $\sigma$ and $\sigma_{i}$ on one hand, $\rho_{*}, v^{i}$, and $s$ on the other hand, is determined by the form of the stress-energy tensor. We shall focus henceforth on adiabatic fluid systems, for which

$$
T^{\mu \nu}=\rho\left(c^{2}+h\right) u^{\mu \nu}+p g^{\mu \nu},
$$

where $h$ and $p$ stand for the enthalpy per unit mass and for the pressure respectively. The stress-energy tensor (4.5) has to be supplemented by the equation of state of the fluid; it is typically provided by the energy $e$ per unit mass $^{1}$ as a function of the scalar density and of the entropy, $e=e(\rho, s)$, from what we can infer the pressure $p(\rho, s)=\rho^{2} \partial e(\rho, s) / \partial \rho$ or the enthalpy $h(\rho, s)=e(\rho, s)+$ $p(\rho, s) / \rho$. It is now possible to relate the densities $\sigma$ to $\rho_{*}$, $v^{i}$, and $s$. At the Newtonian approximation, we have $\sigma=$ $\left(T^{00}+T^{i i}\right) / c^{2}=\left[\rho c^{2} u^{0^{2}}+\mathcal{O}\left(1 / c^{0}\right)\right] / c^{2}=\rho_{*}+\mathcal{O}\left(1 / c^{2}\right)$,

\footnotetext{
${ }^{1}$ Let us point out that the symbol $e$ denotes the energy per volume unit in paper [5]
}

and $\sigma_{i}=T^{0 i} / c=\left[\rho c^{2} u^{i} u^{0}+\mathcal{O}(1 / c)\right] / c=\rho_{*} v^{i}+\mathcal{O}\left(1 / c^{2}\right)$. It is thus useful to pose:

$$
\begin{aligned}
U_{*} & =G \int \frac{d^{3} \mathbf{y}}{|\mathbf{x}-\mathbf{y}|} \rho_{*}, \\
U_{* i} & =G \int \frac{d^{3} \mathbf{y}}{|\mathbf{x}-\mathbf{y}|} \rho_{*} v^{i}, \\
\chi_{*} & =G \int d^{3} \mathbf{y}|\mathbf{x}-\mathbf{y}| \rho_{*} .
\end{aligned}
$$

At the order $1 \mathrm{PN}$ and beyond we proceed in several steps, for the computations are longer. We first expand $\sqrt{-g^{\prime}}$ in power of $1 / c$ in order to evaluate $\rho\left(\rho_{*}, v^{p}, s\right)=$ $\rho_{*} /\left(u^{0} \sqrt{-g^{\prime}}\right)$. From the expression of the determinant of a perturbed Minkowski metric $g_{\mu \nu}^{\prime}=\eta_{\mu \nu}+\delta g_{\mu \nu}^{\prime}$ at linear order, $g^{\prime}=-1+\delta g^{\prime}+\mathcal{O}\left(\left(\delta g^{\prime}\right)^{2}\right)$ with $\delta g^{\prime}=$ $\operatorname{det}\left(\eta_{\alpha \beta}\right) \eta^{\rho \sigma} \delta g_{\rho \sigma}^{\prime}=-\eta^{\alpha \beta} \delta g_{\alpha \beta}^{\prime}$, we get immediately

$$
\begin{aligned}
\sqrt{-g^{\prime}}= & \left(1+\eta^{\alpha \beta} \delta g_{\alpha \beta}^{\prime}\right)^{1 / 2}+\mathcal{O}\left(\left(\delta g^{\prime}\right)^{2}\right) \\
= & 1-\frac{1}{2}\left(g_{00}^{\prime}\right)_{(\leq 7)}+\frac{1}{2}\left(g_{i i}^{\prime}\right)_{(\leq 7)}+\mathcal{O}\left(\frac{1}{c^{2}}\right) \mathcal{O}\left(g_{\text {odd }}^{\prime}\right) \\
& +\frac{1}{c^{4}}(\ldots)+\frac{1}{c^{6}}(\ldots)+\mathcal{O}\left(\frac{1}{c^{8}}\right) .
\end{aligned}
$$

The lowest contribution to $\mathcal{O}\left(1 / c^{2}\right) \mathcal{O}\left(g_{\text {odd }}^{\prime}\right)$ is a Lorentz scalar proportional to $\left(g_{00}^{\prime}\right)_{(2)}$ or $\left(g_{i j}^{\prime}\right)_{(2)}$, and $\left(g_{i j}^{\prime}\right)_{(5)}$. Now, the only allowed combinations are $\left(g^{\prime \alpha \beta}\right)_{(2)} \times$ $\left(g_{\alpha \beta}^{\prime}\right)_{(5)} \propto \delta^{i j} g_{i j(5)}^{\prime}$, and $\eta^{\alpha \beta}\left(g_{\alpha \beta}^{\prime}\right)_{(2)} \eta^{\rho \sigma}\left(g_{\rho \sigma}^{\prime}\right)_{(5)}$, which are both zero due to the trace-free property of $\left(g_{i j}^{\prime}\right)_{(5)}=$ $-4 G I_{i j}^{(3)} /\left(5 c^{5}\right)$. As a consequence,

$$
\begin{array}{r}
\sqrt{-g^{\prime}}=1+\frac{2}{c^{2}} U-\frac{1}{2}\left(g_{00}^{\prime}\right)_{(7)}+\frac{1}{2}\left(g_{i i}^{\prime}\right)_{(7)}+\frac{1}{c^{4}}(\ldots) \\
+\frac{1}{c^{6}}(\ldots)+\mathcal{O}\left(\frac{1}{c^{8}}\right) .
\end{array}
$$

We next calculate the Lorentz factor $u^{0}=d t / d \tau$ appearing in $\rho$ and $v^{i}$, from the definition of the proper time $c d \tau=\left(-g_{\alpha \beta}^{\prime} d x^{\alpha} d x^{\beta}\right)^{1 / 2}$. We find:

$$
\begin{aligned}
u^{0}= & {\left[-g_{00}^{\prime}-2 g_{0 i}^{\prime} \frac{v^{i}}{c}-g_{i j}^{\prime} \frac{v^{i} v^{j}}{c^{2}}\right]^{-1 / 2} } \\
= & {\left[1-\frac{2}{c^{2}} U-\left(g_{00}^{\prime}\right)_{(7)}-2\left(g_{0 i}^{\prime}\right)_{(6)} \frac{v^{i}}{c}-\frac{v^{2}}{c^{2}}\right.} \\
& \left.-\left(g_{i j}^{\prime}\right)_{(5)} \frac{v^{i} v^{j}}{c^{2}}+\frac{1}{c^{4}}(\ldots)+\frac{1}{c^{6}}(\ldots)+\mathcal{O}\left(\frac{1}{c^{8}}\right)\right]^{-1 / 2} \\
= & 1+\frac{U}{c^{2}}+\frac{1}{2} \frac{v^{2}}{c^{2}}+\frac{1}{2}\left(g_{00}^{\prime}\right)_{(7)}+\frac{1}{2}\left(g_{i j}^{\prime}\right)_{(5)} \frac{v^{i} v^{j}}{c^{2}} \\
& +\frac{1}{c^{4}}(\ldots)+\frac{1}{c^{6}}(\ldots)+\mathcal{O}\left(\frac{1}{c^{8}}\right)
\end{aligned}
$$

where $v$ denotes the Euclidean norm of $v^{i}$, i.e. $v^{2}=v^{i} v^{i}$. Regarding the fact that the lowest odd order part of the matter or thermodynamical quantities involved in 
the stress-energy tensor 4.5 is necessarily larger than five, equations (4.7) and (4.8) show that the first odd terms in power of $1 / c$ entering the $1 / c$ expansion of $u^{0}=u^{0}\left(\rho_{*}, v^{p}, s\right)$ and $\rho=\rho\left(\rho_{*}, v^{p}, s\right)$ arise exactly at order seven, which implies that the $1 / c^{5}$ parts of the scalar density and the Lorentz factor are both zero. Referring to these two parts as $u_{(5) \text { reac }}^{0}$ and $\rho_{(5) \text { reac re- }}$ spectively, so as to distinguish them from the fifth order of $\rho$ and $u^{i}$ considered as functions of $\sigma, \sigma_{i}$, and $\sigma_{i j}$, we can write in short: $u_{(5) \text { reac }}^{0}=\rho_{(5) \text { reac }}=0$. Therefore, the densities $\sigma=\rho\left(u^{0}\right)^{2}+\mathcal{O}\left(1 / c^{2}\right)$ and $\sigma_{i}=$ $\rho\left(u^{0}\right)^{2} v^{i}+\mathcal{O}\left(1 / c^{2}\right)$ have no contribution of order $1 / c^{5}$, i.e. $\sigma_{(5) \text { reac }}=0$ and $\sigma_{i(5) \text { reac }}=0$ (with the same notation as before). Let us mention that the stress density $\sigma_{i j}=\rho\left(u^{0}\right)^{2} v^{i} v^{j}+p g^{i j}+\mathcal{O}\left(1 / c^{2}\right)$ does contribute at this level:

$$
\begin{aligned}
\sigma_{(5) i j \text { reac }} & =p\left(\rho_{*}, s\right)\left(g^{i j}\right)_{(5) \text { reac }} \\
& =-p\left(\rho_{*}, s\right)\left(g_{i j}^{\prime}\right)_{(5) \text { reac }} \neq 0 .
\end{aligned}
$$

The fact that we have $U_{(5) \text { reac }}=\chi_{(5) \text { reac }}=0$ and $U_{i(5) \text { reac }}=0$ for the potentials leads us to conclude that the reaction part of the gravitational field is simply

$$
\begin{aligned}
& \left(g_{00}^{\prime}\right)_{(7) \text { reac }}=\left[-1+\frac{2}{c^{2}} U+\frac{1}{c^{4}}(\ldots)\right. \\
& \left.+\frac{1}{c^{6}}(\ldots)+\left(g_{00}^{\prime}\right)_{(7)}\right]_{\text {reac }}=\left[\left(g_{00}^{\prime}\right)_{(7)}\right]_{\text {reac }} \\
& =\frac{4 G}{5 c^{7}}\left(-Q_{k l}^{(3)} x^{k} \partial_{l} U_{*}\right. \\
& \left.+G \int \frac{d^{3} \mathbf{y}}{|\mathbf{x}-\mathbf{y}|} Q_{k l}^{(3)} x^{k} \partial_{l} \rho_{*}\right) \\
& \left(g_{0 i}^{\prime}\right)_{(6) \text { reac }}=\left[-\frac{1}{c^{3}}\left(4 U_{i}+\frac{1}{2} \partial_{i} \partial_{t} \chi\right)\right. \\
& \left.+\frac{1}{c^{5}}(\ldots)+\left(g_{0 i}^{\prime}\right)_{(6)}\right]_{(6) \mathrm{reac}}=0 \\
& \left(g_{i j}^{\prime}\right)_{(5) \text { reac }}=\left[\delta^{i j}\left(1+\frac{2}{c^{2}} U\right)+\frac{1}{c^{4}}(\ldots)+\left(g_{i j}^{\prime}\right)_{(5)}\right]_{\text {reac }} \\
& =\left[\left(g_{i j}^{\prime}\right)_{(5)}\right]_{(5) \text { reac }}=-\frac{4 G}{5 c^{5}} Q_{i j}^{(3)}
\end{aligned}
$$

at the leading approximation. The functions $\left[\left(g_{00}^{\prime}\right)_{(7)}\right]_{(7) \text { reac }}$ and $\left[\left(g_{i j}^{\prime}\right)_{(5)}\right]_{(5) \text { reac}}$, are obtained by replacing consistently the Newtonian-like potentials $U, U_{i}, \chi$, and the mass quadrupole moment $I_{i j}$, by $U_{*}, U_{* i}, \chi_{*}$, and $Q_{i j}=\int d^{3} \mathbf{y} \hat{y}^{i j} \rho_{*}$ respectively in the corresponding odd metric components. From equations (4.7), 4.8), and (4.10), we deduce $u^{0}$ and $\rho$ expressed within our set of variables:

$$
u^{0}=1+\frac{U_{*}}{c^{2}}+\frac{1}{2} \frac{v^{2}}{c^{2}}+\frac{1}{2}\left[\left(g_{00}^{\prime}\right)_{(7)}\right]_{(7) \text { reac }}
$$

$$
\begin{aligned}
& +\frac{1}{2}\left[\left(g_{i j}^{\prime}\right)_{(5)}\right]_{\text {reac }} \frac{v^{i} v^{j}}{c^{2}}+\frac{1}{c^{4}}(\ldots)+\frac{1}{c^{6}}(\ldots)+\mathcal{O}\left(\frac{1}{c^{8}}\right) \\
& \rho=\rho_{*}\left(1-\frac{3}{c^{2}} U_{*}-\frac{1}{2} \frac{v^{2}}{c^{2}}\right) \\
& -\frac{\rho_{*}}{2}\left(\left[\left(g_{i i}^{\prime}\right)_{(7)}\right]_{(7) \text { reac }}+\left[\left(g_{i j}^{\prime}\right)_{(5)}\right]_{(5) \text { reac }} \frac{v^{i} v^{j}}{c^{2}}\right) \\
& +\frac{1}{c^{4}}(\ldots)+\frac{1}{c^{6}}(\ldots)+\mathcal{O}\left(\frac{1}{c^{8}}\right) .
\end{aligned}
$$

At last, we compute the pressure $p$ as a function of $\rho_{*}$, $v^{i}$, and $s$, by expanding $p\left(\rho_{*}+\delta \rho, s\right)=\left(\rho_{*}+\delta \rho\right)^{2} \partial e\left(\rho_{*}+\right.$ $\delta \rho, s) / \partial \rho$ around $\rho=\rho_{*}$. According to relation (4.12), the difference $\delta \rho \equiv \rho-\rho_{*}$ splits into (i) a "conservative" term of order $1 / c^{2}$, which we shall note $\rho_{(2) \text { cons }}$ henceforth, (ii) a "reaction" term of order $1 / c^{7}$, plus (iii) contributions of order $1 / c^{4}, 1 / c^{6}$, and $\mathcal{O}\left(1 / c^{8}\right)$ that are irrelevant in our calculation. Thus, $\mathcal{O}\left([\delta \rho]^{2}\right)=$ $1 / c^{4}(\ldots)+1 / c^{6}(\ldots)+\mathcal{O}\left(1 / c^{8}\right)$, and the quadratic term of the Taylor series can be omitted.

$$
\begin{aligned}
p\left(\rho_{*}+\delta \rho, s\right)= & p\left(\rho_{*}, s\right)+\delta \rho \frac{\partial p}{\partial \rho}\left(\rho_{*}, s\right)+\mathcal{O}\left([\delta \rho]^{2}\right) \\
= & p\left(\rho_{*}, s\right)+\left(\rho_{(2)}+\rho_{(7)}\right) \frac{\partial p}{\partial \rho}\left(\rho_{*}, s\right) \\
& +\frac{1}{c^{4}}(\ldots)+\frac{1}{c^{6}}(\ldots)+\mathcal{O}\left(\frac{1}{c^{8}}\right) .
\end{aligned}
$$

We define the coordinate pressure $p_{*}$ to be the pressure of a fictitious fluid of scalar density $\rho_{*}$ and of entropy $s$ in our coordinate grid: $p_{*} \equiv p\left(\rho_{*}, s\right)$. Its numerical value a priori differs from that of the actual pressure $p \equiv p\left(\rho\left(\rho_{*}, v^{p}, s\right), s\right)$. We introduce in the same way a coordinate enthalpy $h_{*}$ as well as a coordinate adiabatic index $\gamma_{*}=\partial \ln p_{*} / \partial \ln \rho_{*}$, etc. Whereas $h \neq h_{*}$ and $\gamma \neq$ $\gamma_{*}$ in general, $\gamma_{*}$ identifies with the usual adiabatic index $\gamma=\partial \ln p / \partial \ln \rho$ when the equation of state is assumed to be polytropic. Though $\gamma_{*}$ happens to be constant in the latter case, it usually depends on time and on the field point: $\gamma_{*}=\gamma_{*}(\mathbf{x}, t)$. Within the preceding notation, equation (4.13) becomes:

$$
\begin{aligned}
p= & p_{*}\left[1-\frac{\gamma_{*}}{c^{2}}\left(3 U_{*}+\frac{1}{2} v^{2}\right)\right] \\
& -\frac{p_{*} \gamma_{*}}{2}\left(\left[\left(g_{i i}^{\prime}\right)_{(7)}\right]_{(7) \text { reac }}+\left[\left(g_{i j}^{\prime}\right)_{(5)}\right]_{(5) \text { reac }} \frac{v^{i} v^{j}}{c^{2}}\right) \\
& +\frac{1}{c^{4}}(\ldots)+\frac{1}{c^{6}}(\ldots)+\mathcal{O}\left(\frac{1}{c^{8}}\right) .
\end{aligned}
$$

This entails notably that $h=h_{*}+(\ldots) / c^{2}+(\ldots) / c^{4}+$ $\mathcal{O}\left(1 / c^{6}\right)$. Finally, the $1 \mathrm{PN}+3.5 \mathrm{PN}$ mass density $\sigma=$ $\left(T^{00}+T^{i i}\right) / c^{2}$ is derived by inserting expressions (4.3), (4.11), (4.12), and (4.14) into the stress-energy tensor $T^{\mu \nu}=\rho_{*} u^{0}\left(1+h / c^{2}\right) v^{\mu} v^{\nu} / \sqrt{-g^{\prime}}+p g^{\prime \mu \nu}$. We arrive at

$$
\sigma=\rho_{*}+\frac{1}{c^{2}}\left[\rho_{*} c^{2}\left(1+u_{(2) \text { cons }}^{0}+u_{(7) \text { reac }}^{0}\right)\right.
$$




$$
\begin{aligned}
&\left.\times\left(1-\left(\sqrt{-g^{\prime}}\right)_{(2) \text { cons }}-\left(\sqrt{-g^{\prime}}\right)_{(7) \text { reac }}\right)-p_{*}\right] \\
&+\frac{1}{c^{2}}\left[\rho_{*} v^{2}+p_{*}\left(\delta^{i i}-\left[\left(g^{\prime i i}\right)_{(5)}\right]_{(5) \text { reac }}\right)\right] \\
&=\rho_{*}+\frac{\rho_{*}}{c^{2}}\left(\frac{3}{2} v^{2}-U_{*}+h_{*}+2 \frac{p_{*}}{\rho_{*}}\right)+\rho_{*}\left(\left[\left(g_{00}^{\prime}\right)_{(7)}\right]_{\text {reac }}\right. \\
&\left.-\frac{1}{2}\left[\left(g_{i i}^{\prime}\right)_{(7)}\right]_{(7) \text { reac }}+\frac{1}{2}\left[\left(g_{i j}^{\prime}\right)_{(5)}\right]_{(5) \text { reac }} \frac{v^{i} v^{j}}{c^{2}}\right),
\end{aligned}
$$

noticing that $\left[\left(g^{\prime i i}\right)_{(5)}\right]_{(5) \text { reac }}=-\left[\left(g_{i i}^{\prime}\right)_{(5)}\right]_{(5) \text { reac }}+$ $\mathcal{O}\left(1 / c^{7}\right)=\mathcal{O}\left(1 / c^{7}\right)$. The Poisson integral of the above expansion gives the potential $U$ in the $\left\{\rho_{*}, v^{i}, s\right\}$ representation, modulo an unimportant prefactor $G$.

$$
\begin{aligned}
U= & U_{*}+\frac{G}{c^{2}} \int \frac{d^{3} \mathbf{y}}{|\mathbf{x}-\mathbf{y}|} \rho_{*}\left(\frac{3}{2} v^{2}-U_{*}+h_{*}+2 \frac{p_{*}}{\rho_{*}}\right) \\
+ & G \int \frac{d^{3} \mathbf{y}}{|\mathbf{x}-\mathbf{y}|} \rho_{*}\left(\left[\left(g_{00}^{\prime}\right)_{(7)}\right]_{\text {reac }}-\frac{1}{2}\left[\left(g_{i i}^{\prime}\right)_{(7)}\right]_{\text {reac }}\right. \\
& \left.+\frac{1}{2}\left[\left(g_{i j}^{\prime}\right)_{(5)}\right]_{(5) \text { reac }} \frac{v^{i} v^{j}}{c^{2}}\right) \\
& +\frac{1}{c^{4}}(\ldots)+\frac{1}{c^{6}}(\ldots)+\mathcal{O}\left(\frac{1}{c^{8}}\right) .
\end{aligned}
$$

All other potentials can be cast into a similar form. The leading orders of the conservative and reaction part are determined by that of $\sigma$ and $\sigma_{i}$. As the fifth order corrections of both mass and current density vanish, we have $\chi=\chi_{*}+\chi_{(2) \text { cons }}+\chi_{(7) \text { reac }}$ and $U_{i}=$ $U_{* i}+U_{i(2) \text { cons }}+U_{i(7) \text { reac }}$, modulo irrelevant terms.

We are now in measure to isolate the "reaction" field $\left(g_{\mu \nu}^{\prime}\right)_{\mathrm{reac}}$. It essentially consists of the odd metric 4.3) to which add the reaction terms generated by the postNewtonian part of $\left(g_{\mu \nu}^{\prime}\right)_{\text {even }}=g_{\mu \nu}^{\prime}-\left(g_{\mu \nu}^{\prime}\right)_{\text {odd }}$ regarded as a function of the matter variables $\sigma$ and $\sigma_{i}$. The latter terms do not contribute to $\left(g_{0 i}^{\prime}\right)_{\text {reac }}$ or $\left(g_{i j}^{\prime}\right)_{\text {reac }}$ at the 3.5 PN order, but enter the 00 component of $\left(g_{\mu \nu}^{\prime}\right)_{\text {reac }}$,

$$
\begin{aligned}
\left(g_{00}^{\prime}\right)_{\text {reac }} & =\left(g_{00}^{\prime}\right)_{\text {odd }}+\frac{2}{c^{2}} U_{(7) \text { reac }}+\mathcal{O}\left(\frac{1}{c^{10}}\right), \\
\left(g_{0 i}^{\prime}\right)_{\text {reac }} & =\left(g_{0 i}^{\prime}\right)_{\text {odd }}-\frac{1}{c^{3}} A_{i(7) \text { reac }}+\mathcal{O}\left(\frac{1}{c^{9}}\right) \\
& =\left(g_{0 i}^{\prime}\right)_{\text {odd }}+\mathcal{O}\left(\frac{1}{c^{9}}\right), \\
\left(g_{i j}^{\prime}\right)_{\text {reac }} & =\left(g_{i j}^{\prime}\right)_{\text {odd }}+\delta^{i j} \frac{2}{c^{2}} U_{(7) \text { reac }}+\mathcal{O}\left(\frac{1}{c^{8}}\right) \\
& =\left(g_{i j}^{\prime}\right)_{\text {odd }}+\mathcal{O}\left(\frac{1}{c^{8}}\right) .
\end{aligned}
$$

By virtue of equations (4.3), (4.4), 4.16), and (4.17), we finally obtain the 2.5 and $3.5 \mathrm{PN}$ components of the metric as

$$
\begin{aligned}
\left(g_{00}^{\prime}\right)_{(9) \text { reac }}= & \frac{4 G}{5 c^{9}}\left(-I_{2}^{(3)} x^{k} \partial_{l} U_{*}+Q_{k l}^{(3)} x^{k}\left(-\partial_{l} U_{2}+2 U_{*} \partial_{l} U_{*}\right)+Q_{k l}^{(4)} x^{k}\left(-\frac{1}{2} x^{l} \partial_{t} U_{*}+A_{* l}\right)+\frac{5}{126} Q_{k l m}^{(5)} x^{k} x^{l} \partial_{m} U_{*}\right. \\
& +Q_{k l}^{(5)} x^{k}\left(\frac{17}{42} x^{l} x^{m} \partial_{m} U_{*}-\frac{11}{42} r^{2} \partial_{l} U_{*}\right)-\frac{8}{9} \epsilon_{k l m} S_{m n}^{(4)} x^{l} x^{n} \partial_{k} U_{*}-2 U G \int \frac{d^{3} \mathbf{y}}{|\mathbf{x}-\mathbf{y}|} Q_{k l}^{(3)} y^{k} \partial_{l} \rho_{*} \\
& +G \int \frac{d^{3} \mathbf{y}}{|\mathbf{x}-\mathbf{y}|}\left[I_{2 k l}^{(3)} y^{k} \partial_{l} \rho_{*}+Q_{k l}^{(3)} y^{k}\left(\rho_{*} \partial_{l} U_{*}+\partial_{l}\left(\rho_{*} \delta\right)\right)-3 \rho_{*} v^{k} v^{l} Q_{k l}^{(3)}-\frac{5}{126} Q_{k l m}^{(5)} y^{k} y^{l} \partial_{m} \rho_{*}\right. \\
& +Q_{k l}^{(4)} y^{k}\left(\frac{1}{2} y^{l} \partial_{t} \rho_{*}-4 \rho_{*} v^{l}\right)+Q_{k l}^{(5)} y^{k}\left(-\frac{17}{42} y^{l} y^{m} \partial_{m} \rho_{*}+\frac{11}{42}|\mathbf{y}|^{2} \partial_{l} \rho_{*}-\rho_{*} y^{l}\right)-\frac{8}{9} \epsilon_{k l m} S_{m n}^{(4)} y^{l} y^{n} \partial_{k} \rho_{*} \\
& \left.\left.-G \rho_{*} \int \frac{d^{3} \mathbf{y}^{\prime}}{\left|\mathbf{y}-\mathbf{y}^{\prime}\right|}\left(Q_{k l}^{(3)} y^{k} \partial_{l} \rho_{*}\right)\left[\mathbf{y}^{\prime}\right]\right]\right) \\
\left(g_{0 i}^{\prime}\right)_{(8) \text { reac }}= & -\frac{8 G}{5 c^{8}}\left(-\frac{1}{4} Q_{k l}^{(3)} x^{k} \partial_{l} A_{* i}-\frac{1}{4} Q_{i k}^{(3)} A_{* k}+Q_{i k}^{(4)} x^{k} U_{*}+\frac{G}{8} \partial_{i} \partial_{t} \int d^{3} \mathbf{y}|\mathbf{x}-\mathbf{y}|\left[Q_{k l}^{(3)} y^{k} \partial_{l} \rho_{*}\right]\right. \\
& \left.+G \int \frac{d^{3} \mathbf{y}}{|\mathbf{x}-\mathbf{y}|}\left[Q_{k l}^{(3)} y^{k} \partial_{l}\left(\rho_{*} v^{i}\right)-\rho_{*} v^{k} Q_{i k}^{(3)}-\rho_{*} y^{k} Q_{i k}^{(4)}\right]\right) \\
\left(g_{i j}^{\prime}\right)_{(7) \text { reac }}= & \frac{4 G}{5 c^{7}}\left(-I_{2 i j}^{(3)}-2 Q_{i j}^{(3)} U_{*}+\frac{5}{63} Q_{i j k}^{(5)} x^{k}+\frac{2}{7} x^{k} x^{(i} Q_{j) k}^{(5)}-\frac{11}{42} Q_{i j}^{(5)} r^{2}-\frac{8}{9} \epsilon_{k l(i} S_{j) l}^{(4)} x^{k}\right) \\
& +\frac{4 G}{5} \delta^{i j}\left(-\frac{2}{21} x^{k} x^{l} Q_{k l}^{(5)}-Q_{k l}^{(3)} x^{k} \partial_{l} U_{*}+G \int \frac{d^{3} \mathbf{y}}{|\mathbf{x}-\mathbf{y}|} Q_{k l}^{(3)} y^{k} \partial_{l} \rho_{*}\right)
\end{aligned}
$$

with $A_{* i} \equiv 4 U_{* i}+\partial_{i} \partial_{t} \chi_{*} / 2, \quad U_{2} \equiv c^{2} U_{(2) \text { cons },}, \quad \int d^{3} \mathbf{y} \epsilon_{k m<i} \hat{y}^{j>k} \rho_{*} v^{m}$. $I_{2 i j} \equiv c^{2}\left(I_{i j}\right)_{(2) \text { cons }}$, and $S_{i j} \equiv J_{i j(0) \text { cons }}=$ 


\subsection{PN reaction force}

The evolution equations including the $3.5 \mathrm{PN}$ gravitational damping are deduced from the conservation of the stress-energy tensor

$$
\partial_{\alpha}\left(\sqrt{-g^{\prime}} T_{\mu}^{\alpha}\right)=\frac{1}{2} \sqrt{-g^{\prime}} T^{\alpha \beta} \partial_{\mu} g_{\alpha \beta}^{\prime}
$$

and from that of the entropy

$$
\partial_{t} s+v^{i} \partial_{i} s=0
$$

The projection of formula (4.19) on $u^{\mu}$, together with formula (4.20), leads to the continuity equation

$$
\partial_{t} \rho_{*}+\partial_{i}\left(\rho_{*} v^{i}\right)=0
$$

whereas its space part is equivalent to the Euler equation

$$
\begin{array}{r}
\partial_{j}\left[\rho_{*}\left(1+\frac{h}{c^{2}}\right) u_{i} v^{j} c\right]+\partial_{t}\left[\rho_{*}\left(1+\frac{h}{c^{2}}\right) u_{i} c\right] \\
+\partial_{i}\left(\sqrt{-g^{\prime}} p\right)=\frac{1}{2} \sqrt{-g^{\prime}} T^{\alpha \beta} \partial_{i} g_{\alpha \beta}^{\prime}
\end{array}
$$

In the absence of coupling between radiative and nonradiative effects, the $1 \mathrm{PN}+3.5 \mathrm{PN}$ dynamics of the adiabatic fluid subjected to gravitational damping is described by the post-Newtonian expansion of equalities (4.20), 4.21), (4.22), including the Newtonian, $1 \mathrm{PN}$, $2.5 \mathrm{PN}$, as well as the $3.5 \mathrm{PN}$ order for fixed $\rho_{*}, v^{i}$, and $s$.

We first identify the momentum density of the fluid ${ }^{2}$ $M_{i} \equiv \rho_{*}\left(1+h / c^{2}\right) u_{i} c=\sqrt{-g^{\prime}} T_{i}^{0} / c$ on the left-hand side of (4.22). The space part $u_{i}$ of the covariant velocity is related to the variables $\rho_{*}, v^{i}$, and $s$ through the equality $u_{i}=v^{i} / c+\left[u_{0}\left(g_{i 0}^{\prime}+g_{i j}^{\prime} v^{j} / c\right)\right]_{(3+6+8)}+(\ldots) / c^{5}+(\ldots) / c^{7}+$ $\mathcal{O}\left(1 / c^{9}\right)$. After replacing $u^{0}$ by its value (4.11) in the definition of $M_{i}$ and expanding up to the $1 \mathrm{PN}+3.5 \mathrm{PN}$ order with the help of equations (4.3), we arrive at

$$
\begin{aligned}
M_{i}= & \rho_{*} v^{i}+\frac{\rho_{*}}{c^{2}}\left(h_{*} v^{i}+3 U_{*} v^{i}-A_{* i}+\frac{1}{2} v^{2} v^{i}\right) \\
& +\rho_{*} v^{j}\left(g_{i j}^{\prime}\right)_{(5+7) \text { reac }} \\
& +\frac{1}{c^{2}} \rho_{*}\left(h_{*}+\frac{1}{2} v^{2}+U_{*}\right) v^{j}\left(g_{i j}^{\prime}\right)_{(5) \text { reac }} \\
& +\rho_{*} c\left(g_{0 i}^{\prime}\right)_{(8) \text { reac }}
\end{aligned}
$$

2 Note that the momentum density $M_{i}$ is referred to as $\pi_{i}$ in paper [5]

$$
\begin{aligned}
& +\frac{1}{2} \rho_{*} v^{i}\left(\left(g_{00}^{\prime}\right)_{(7) \text { reac }}+\left(g_{k l}^{\prime}\right)_{(5) \text { reac }} \frac{v^{k} v^{l}}{c^{2}}\right) \\
& +\frac{1}{c^{4}}(\ldots)+\frac{1}{c^{6}}(\ldots)+\mathcal{O}\left(\frac{1}{c^{8}}\right) .
\end{aligned}
$$

This expression contains the term $\rho_{*}\left(g_{i j}^{\prime}\right)_{(5+7)} v^{j}$ involving in particular the third order time derivative of the $1 \mathrm{PN}$ quadrupole $I_{i j}$ and the fifth time derivative of the Newtonian mass multipole moments $Q_{i j}$ and $Q_{i j k}$. Therefore, the extra time derivation appearing on the left-hand side of the Euler equation (4.22) induces terms in $I_{i j(2) \text { cons }}^{(4)}, Q_{i j}^{(6)}$, and $Q_{i j k}^{(6)}$, which make us lose all benefit from the gauge transformation. In order to cure that, we modify our system of variables according to paper [5]. We keep the baryonic density as well as the entropy, but substitute to the coordinate velocity $v^{i}$ the more appropriate "momentum velocity" $w_{i} \equiv M_{i} / \rho_{*}$. The relation between $w_{i}$ and $v^{i}$ is found by inverting equation (4.23) iteratively. At the leading order, we have $M_{i}=\rho_{*} v^{i}+\mathcal{O}\left(1 / c^{2}\right)$, hence $w_{i}=v^{i}+\mathcal{O}\left(1 / c^{2}\right)$. This result is used to compute the $1 \mathrm{PN}$ correction of $v^{i}$ as a function of $w_{i}: M_{i}=$ $\rho_{*} v^{i}-\rho_{*}\left(-h_{*} w_{i}-3 U_{*} w_{i}+A_{* i}-w^{2} w_{i} / 2\right) / c^{2}+\mathcal{O}\left(1 / c^{4}\right)$, hence $v^{i}$, which is in its turn inserted into equation (4.23) truncated at the next approximation, etc. We get in the end:

$$
\begin{aligned}
v^{i} & =w_{i}+\frac{1}{c^{2}}\left(-h_{*} w_{i}-3 U_{*} w_{i}+A_{* i}-\frac{1}{2} w^{2} w_{i}\right) \\
& -w_{j}\left(g_{i j}^{\prime}\right)_{(5) \text { reac }}-w_{j}\left(g_{i j}^{\prime}\right)_{(7) \text { reac }} \\
& +\frac{1}{c^{2}}\left(5 U_{*}+\frac{1}{2} w^{2}+h_{*}\right) w_{j}\left(g_{i j}^{\prime}\right)_{\text {cons }}-\frac{A_{* j}}{c^{2}}\left(g_{i j}^{\prime}\right)_{\text {reac }}^{(5)} \\
& -c\left(g_{0 i}^{\prime}\right)_{\text {reac }}+\frac{w_{i}}{2}\left(-\left(g_{00}^{\prime}\right)_{\text {reac }}+\left(g_{k l}^{\prime}\right)_{\text {reac }} \frac{w_{k} w_{l}}{c^{2}}\right) \\
& +\frac{1}{c^{4}}(\ldots)+\frac{1}{c^{6}}(\ldots)+\mathcal{O}\left(\frac{1}{c^{8}}\right) .
\end{aligned}
$$

Up to now, we have reduced the kinetic part of the Euler equation (4.22) to $\partial_{j}\left(\rho_{*} w_{i} v^{j}\right)+\partial_{t}\left(\rho_{*} w_{i}\right)$, or equivalently $\rho_{*}\left(v^{j} \partial_{j} w_{i}+\partial_{t} w_{i}\right)$, after the continuity equation. The pressure force $F_{i}^{\text {press }} \equiv-\partial_{i}\left(\sqrt{-g^{\prime}} p\right) / \rho_{*}$, as for it, is derived from the post-Newtonian expansion (4.14) and (4.7) of $p$ and $\sqrt{-g^{\prime}}$ respectively, supplemented by the two approximate equalities $U=U_{*}+$ $(\ldots) / c^{2}+(\ldots) / c^{4}+\mathcal{O}\left(1 / c^{6}\right),\left(g_{i i}^{\prime}\right)_{(7)}=\left(g_{i i}^{\prime}\right)_{(7) \text { reac }}$, and $v^{i}=w_{i}-w_{j}\left(g_{i j}^{\prime}\right)_{(5) \text { reac }}+$ irrelevant terms.

$$
\begin{aligned}
& F_{i}^{\mathrm{press}}=-\frac{1}{\rho_{*}} \partial_{i}\left\{p_{*}+\frac{1}{c^{2}}\left[2 U_{*}-\gamma_{*}\left(3 U_{*}+\frac{1}{2} v^{2}\right)\right] p_{*}\right. \\
& \left.+\frac{p_{*}}{2}\left[\left(g_{j j}^{\prime}\right)_{\text {reac }}^{(7)}-\left(g_{00}^{\prime}\right)_{(7) \text { reac }}-\gamma_{*}\left(\left(g_{j j}^{\prime}\right)_{\text {reac }}+\left(g_{j k}^{\prime}\right)_{\text {reac }}^{(5)} \frac{v^{j} v^{k}}{c^{2}}\right)\right]\right\}+\frac{1}{c^{4}}(\ldots)+\frac{1}{c^{6}}(\ldots)+\mathcal{O}\left(\frac{1}{c^{8}}\right)
\end{aligned}
$$




$$
\begin{aligned}
=-[1 & \left.+\frac{1}{c^{2}}\left(2 U_{*}-\gamma_{*}\left(3 U_{*}+\frac{1}{2} w^{2}\right)\right)+\frac{1}{2}\left(\left(g_{j j}^{\prime}\right)_{\text {reac }}^{(7)}-\left(g_{00}^{\prime}\right)_{\text {reac }}^{(7)}\right)-\frac{\gamma_{*}}{2}\left(\left(g_{j j}^{\prime}\right)_{\text {reac }}-\left(g_{j k}^{\prime}\right)_{\text {reac }} \frac{w_{j} w_{k}}{c^{2}}\right)\right] \frac{\partial_{i} p_{*}}{\rho_{*}} \\
& -\frac{p_{*}}{\rho_{*}} \partial_{i}\left[\frac{1}{c^{2}}\left(2 U_{*}-\gamma_{*}\left(3 U_{*}+\frac{1}{2} w^{2}\right)\right)+\frac{1}{2}\left(\left(g_{j j}^{\prime}\right)_{(7) \text { reac }}-\left(g_{00}^{\prime}\right)_{(7) \text { reac }}\right)\right. \\
& \left.-\frac{\gamma_{*}}{2}\left(\left(g_{j j}^{\prime}\right)_{(7) \text { reac }}-\left(g_{j k}^{\prime}\right)_{(5) \text { reac }} \frac{w_{j} w_{k}}{c^{2}}\right)\right]+\frac{1}{c^{4}}(\ldots)+\frac{1}{c^{6}}(\ldots)+\mathcal{O}\left(\frac{1}{c^{8}}\right) .
\end{aligned}
$$

While both pressure and density tend toward zero in the region of space-time extending outside the system, the ratio $\partial_{i} p_{*} / \rho_{*}$ remains finite by virtue of the thermodynamical relation $\partial_{i} p_{*} / \rho_{*}=\partial_{i} h_{*}-T_{*} \partial_{i} s$, with $T_{*}=\partial e\left(\rho_{*}, s\right) / \partial s$ representing the coordinate temperature. The last term of the Euler equation still to be evaluated is the gravitational force $F_{i}^{\text {grav }} \equiv \partial_{i} g_{\alpha \beta}^{\prime} \sqrt{-g^{\prime}} T^{\alpha \beta} /\left(2 \rho_{*}\right)$. The components $g_{\mu \nu}^{\prime}$ of the metric in the new gauge and the square root of minus the corresponding determinant are given by equations (4.18) and (4.7) respectively. The stress-energy tensor is obtained from the mass density (4.15), the current density $\sigma_{i}=\rho_{*} v^{i}+\mathcal{O}\left(1 / c^{2}\right)$, as well as the stress density (4.9), by inverting the identities $\sigma=\left(T^{00}+T^{i i}\right) / c^{2}, \sigma_{i}=T^{0 i} / c$, and $\sigma_{i j}=T^{i j}$. All contributions of order $1 / c^{4}, 1 / c^{6}$, and $\mathcal{O}\left(1 / c^{8}\right)$ are neglected as usual. We find:

$$
\begin{aligned}
F_{i}^{\text {grav }}=\frac{1}{\rho_{*}} & \left\{\frac{1}{2} \partial_{i}\left[-1+\frac{2}{c^{2}} U_{*}-\frac{2}{c^{4}} U_{*}^{2}\right]\left[\sqrt{-g^{\prime}}\left(c^{2} \sigma-\sigma_{j j}\right)\right]_{(-2+0)}+\partial_{i}\left[-\frac{A_{* j}}{c^{3}}\right]\left[\sqrt{-g^{\prime}} c \sigma_{j}\right]_{\text {cons }}{ }_{\text {cons }}\right. \\
& +\frac{1}{2} \partial_{i}\left[\delta^{j k}\left(1+\frac{2}{c^{2}} U_{*}\right)\right]\left[\sqrt{-g^{\prime}} \sigma_{j k}\right]_{\text {cons }}+\frac{1}{2} \partial_{i}\left[-1+\frac{2}{c^{2}} U_{*}\right]\left[\sqrt{-g^{\prime}}\left(c^{2} \sigma-\sigma_{j j}\right)\right]_{\text {reac }} \\
& +\partial_{i}\left[-\frac{A_{* j}}{c^{3}}\right]\left[\sqrt{-g^{\prime}} \sigma_{j}\right]_{(4)}+\frac{1}{2} \partial_{i}\left[\delta^{j k}\left(1+\frac{2}{c^{2}} U_{*}\right)\right]\left[\sqrt{-g^{\prime}} \sigma_{j k}\right]_{\text {reac }} \underset{\text { reac }}{ } \\
& +\frac{1}{2} \partial_{i}\left(g_{00}^{\prime}\right)_{(7+9)}\left[\sqrt{-g^{\prime}}\left(c^{2} \sigma-\sigma_{j j}\right)\right]_{(-2+0)}+\partial_{i}\left(g_{0 j}^{\prime}\right)_{(6+8)}\left[\sqrt{-g^{\prime}} c \sigma_{j}\right]_{(-1) \text { cons }} \\
& \left.+\frac{1}{2} \partial_{i}\left(g_{j k}^{\prime}\right)_{(5+7)}\left[\sqrt{-g^{\prime}} \sigma_{j k}\right]_{(0+2) \text { cons }}+\frac{1}{c^{4}}(\ldots)+\frac{1}{c^{6}}(\ldots)+\mathcal{O}\left(\frac{1}{c^{8}}\right)\right\} \\
=\partial_{i} & U_{*}\left[1+\frac{1}{c^{2}}\left(\frac{3}{2} v^{2}-U_{*}+h_{*}+2 \frac{p_{*}}{\rho_{*}}\right)\right]+\frac{1}{c^{2}} \partial_{i} U_{2}-\frac{1}{c^{2}} v^{j} \partial_{i} A_{* j} \\
& +\frac{1}{2} c^{2} \partial_{i}\left(g_{00}^{\prime}\right)_{(7) \text { reac }}+\frac{1}{2} \partial_{i} U_{*}\left(\left(g_{00}^{\prime}\right)_{(7) \text { reac }}+\left(g_{j k}^{\prime}\right)_{(5) \text { reac }} \frac{v^{j} v^{k}}{c^{2}}\right) \\
& +\frac{1}{2} \partial_{i}\left(g_{00}^{\prime}\right)_{(7) \text { reac }}\left(h_{*}+U_{*}+\frac{1}{2} v^{2}-\frac{p_{*}}{\rho_{*}}\right)+\frac{c^{2}}{2} \partial_{i}\left(g_{00}^{\prime}\right)_{(9) \text { reac }}+c v^{j} \partial_{i}\left(g_{0 j}^{\prime}\right)_{(8) \text { reac }} \\
& +\frac{1}{2}\left(v^{j} v^{k} \partial_{i}\left(g_{j k}^{\prime}\right)_{(7) \text { reac }}+\frac{p_{*}}{\rho_{*}} \partial_{i}\left(g_{j j}^{\prime}\right)_{(7) \text { reac }}\right)+\frac{1}{c^{4}}(\ldots)+\frac{1}{c^{6}}(\ldots)+\mathcal{O}\left(\frac{1}{c^{8}}\right) .
\end{aligned}
$$

The indetermination of the ratio $p_{*} / \rho_{*}$ for $p_{*}$ and $\rho_{*}$ both going to zero is raised by use of the relation $p_{*} / \rho_{*}=\rho_{*} \partial e\left(\rho_{*}, s\right) / \partial \rho_{*}$. The replacement of $v^{i}$ in $F_{i}^{\text {grav }}$ according to equation (4.24) amounts to (i) changing $v^{i}$ into $w_{i}$ at each of its occurrences, and (ii) adding the $3.5 \mathrm{PN}$ term: $\delta F_{i}^{\text {grav }}=$ $-3 \partial_{i} U_{*} w_{j} w_{k}\left(g_{j k}^{\prime}\right)_{(5) \text { reac }} / c^{2}+\partial_{i} A_{* j} w_{k}\left(g_{j k}^{\prime}\right)_{(5) \text { reac }} / c^{2}$ to the resulting expression. Knowing $v^{i}, F_{i}^{\text {grav }}$, and $F_{i}^{\text {press }}$, we write the Euler equation as:

$$
\partial_{t} w_{i}+v^{j} \partial_{j} w_{i}=F_{i}^{\text {grav }}+F_{i}^{\text {press }}
$$

The evolution equations for the fluid are now established. They are parametrized by the Newtonian potential $U_{*}$, the post-Newtonian potential $A_{* i}=4 U_{* i}+$ $\partial_{i} \partial_{t} \chi_{*} / 2$, as well as the 2.5 and $3.5 \mathrm{PN}$ reaction metric components $\left(g_{00}^{\prime}\right)_{(7) \text { reac }},\left(g_{00}^{\prime}\right)_{(9) \text { reac }},\left(g_{0 i}^{\prime}\right)_{(8) \text { reac }}$,

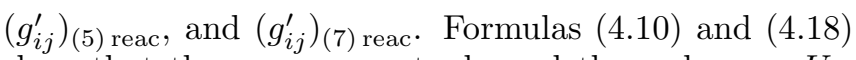
show that these components depend themselves on $U_{*}$, $A_{* i}$, and on the Poisson integrals coming from the functional derivation. Among these various quantities, the only super potential, $\chi_{*}$, appears exclusively through its gradient, $\partial_{i} \chi_{*}=G \int d^{3} \mathbf{y} \rho_{*}\left(x^{i}-y^{i}\right) /|\mathbf{x}-\mathbf{y}|$, which can be put under the form $\partial_{i} \chi_{*}=x^{i} \Delta^{-1}\left(-4 \pi G \rho_{*}\right)-$ $\Delta^{-1}\left(-4 \pi G x^{i} \rho_{*}\right)$. Consequently, all the elementary field quantities employed in the present formulation are solutions of decoupled Poisson equations with compact support sources. This simple elliptic structure fits particularly well to numerical computations. The only complication, apart from that due to the mere length of the expressions, comes from the double integration arising in

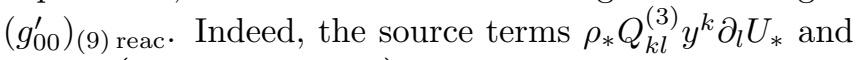
$\rho_{*} \Delta^{-1}\left(-4 \pi G Q_{k l}^{(3)} y^{k} \partial_{l} \rho_{*}\right)$ present in the second member 
of equation 4.18b are basically themselves Poisson potentials. This nonlinear effect originates in the fact that the integrand of $U_{(7) \text { reac }}$ involves certain pieces of the 2.5 and $3.5 \mathrm{PN}$ gravitational field, namely $\left(g_{00}^{\prime}\right)_{(7) \text { reac }}$,

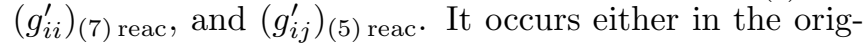
inal Burke-Thorne-like grid or in the coordinate system defined in section 【II as long as we confine ourselves to the $\left\{\rho_{*}, w_{i}, s\right\}$ representation, but can be cured by redefining the density variable as $\tilde{\rho}=\rho_{*}+\sigma_{(7)}$. This results in a slight modification of the present formalism. The corresponding set of equations is presented in Appendix A

Our last task in the achievement of our initial goal will consist in reducing the number of temporal derivatives remaining in the metric (4.18) to one. This will be done by eliminating $\partial_{t} \rho_{*}, \partial_{t} w_{i}$, and $\partial_{t} s$ with the help of the entropy conservation, of the continuity equation, and of the Euler equation at the Newtonian or post-Newtonian order. The multiple time derivatives we shall have to deal with apply on the multipole moments. They are of the type $(d / d t)^{n} \int d^{3} \mathbf{x} \rho_{*}(\mathbf{x}, t) f(\mathbf{x}, t)$, with $n \in\{2,3, \ldots\}$. The function $f$ depends on time through the evolution variables $\rho_{*}, w_{i}$, and $s: f(\mathbf{x}, t)=$ $f\left(\mathbf{x}, \rho_{*}(\mathbf{y}, t), w_{p}(\mathbf{y}, t), s(\mathbf{y}, t)\right)$. Each differentiation is performed by means of the formula:

$$
\begin{aligned}
& \frac{d}{d t} \int d^{3} \mathbf{x} \rho_{*}(\mathbf{x}, t) f(\mathbf{x}, t)=\int d^{3} \mathbf{x} \partial_{t}\left[\rho_{*}(\mathbf{x}, t) f(\mathbf{x}, t)\right] \\
& =\int d^{3} \mathbf{x}\left[-f(\mathbf{x}, t) \partial_{i}\left(\rho_{*}(\mathbf{x}, t) v^{i}(\mathbf{x}, t)\right)+\rho_{*}(\mathbf{x}, t) \partial_{t} f(\mathbf{x}, t)\right] \\
& =\int d^{3} \mathbf{x} \rho_{*}(\mathbf{x}, t) \frac{d f}{d t}(\mathbf{x}, t),
\end{aligned}
$$

where the last equality is obtained by integration by part. We recall that the total derivative $d f / d t=\partial_{t} f+v^{i} \partial_{i} f$ represents the temporal variation of the field $f$ for a fixed particle of fluid. It satisfies the chain rule $d f / d t=$ $\left(d \rho_{*} / d t\right) \partial f / \partial \rho_{*}+\left(d w_{i} / d t\right) \partial f / \partial w_{i}+(d s / d t) \partial f / \partial s$ for any $f=f\left(\rho_{*}, w_{i}, s\right)$. Taking advantage of the Euler equation (4.27), $d w_{i} / d t=F_{i}^{\text {grav }}+F_{i}^{\text {press }}$, as well as the local conservation of mass (4.21), $d \rho_{*} / d t=-\partial_{i}\left(\rho_{*} v^{i}\right)+v^{i} \partial_{i} \rho_{*}=$ $-\rho_{*} \partial_{i} v^{i}$, and entropy (4.20), $d s / d t=0$, we get:

$$
\frac{d f}{d t}=-\frac{\partial f}{\partial \rho_{*}} \rho_{*} \partial_{i} v^{i}+\frac{\partial f}{\partial w_{i}}\left(F_{i}^{\text {grav }}+F_{i}^{\text {press }}\right) .
$$

This shows that the time derivative of a term $\int d^{3} \mathbf{x} \quad \rho_{*}(\mathbf{x}, t) f(\mathbf{x}, t)$ is itself of the type $\int d^{3} \mathbf{x} \quad \rho_{*}(\mathbf{x}, t) g(\mathbf{x}, t)$ with $g=$ $g\left(\mathbf{x}, \rho_{*}(\mathbf{y}, t), w_{p}(\mathbf{y}, t), s(\mathbf{y}, t)\right)$. In this way, the number of time derivatives acting on the initial integral can be brought down to $n-1, n-2$, etc. After $n-1$ iteration, we are left with a unique time derivative as required. The price to pay is an increase of the expression lengths. The evaluation of $I_{i j}^{(3)}$ is particularly tedious since the mass quadrupole $I_{i j}$ is needed at the order $1 / c^{2}$. After specializing the definition (3.3) of $I_{i j}$ to the desired level of approximation, we have

$$
\begin{aligned}
I_{i j}= & \int d^{3} \mathbf{x} \hat{x}^{i j} \rho_{*}\left[1+\frac{1}{c^{2}}\left(\frac{3}{2} w^{2}-U_{*}+h_{*}+2 \frac{p_{*}}{\rho_{*}}\right)\right] \\
& +\frac{1}{14 c^{2}} \frac{d^{2}}{d t^{2}} \int d^{3} \mathbf{x} r^{2} \hat{x}^{i j} \rho_{*}-\frac{20}{21 c^{2}} \frac{d}{d t} \int d^{3} \mathbf{x} \hat{x}^{i j k} \rho_{*} w_{k} \\
& +\mathcal{O}\left(\frac{1}{c^{3}}\right) .
\end{aligned}
$$

We then perform the time derivation along the procedure explained above. The force per mass unit $F_{i}^{\text {grav }}+F_{i}^{\text {press }}$ is limited to the Newtonian order: $d w_{i} / d t=\partial_{i} U_{*}-\partial_{i} p_{*} / \rho_{*}$. We are led to [5]

$$
I_{i j}=E_{i j}+F_{i j}+G_{i j}+\mathcal{O}\left(\frac{1}{c^{3}}\right)
$$

with

$$
\begin{aligned}
E_{i j}=\operatorname{STF} \int d^{3} \mathbf{x} \rho_{*} x^{i} x^{j}\left[1+\frac{1}{c^{2}}\right. & \left(\frac{1}{2} w^{2}-U_{*}\right. \\
& \left.\left.+h_{*}-\frac{p_{*}}{\rho_{*}}\right)\right], \\
F_{i j}=\operatorname{STF} \int d^{3} \mathbf{x} \frac{\rho_{*}}{c^{2}}\left[\frac{11}{21} r^{2} w_{i} w_{j}\right. & -\frac{4}{7} x^{i} x^{k} w_{k} w_{j} \\
& \left.+\frac{4}{21} x^{i} x^{j} w^{2}\right],
\end{aligned}
$$

and

$$
G_{i j}=\operatorname{STF} \int d^{3} \mathbf{x} \frac{\rho_{*}}{c^{2}}\left[\frac{11}{21} r^{2} x^{i} \partial_{j} U_{*}-\frac{17}{21} x^{i} x^{j} x^{k} \partial_{k} U_{*}\right] .
$$

The computation of $I_{i j}^{(2)}$ from equation (4.30a) is long, but straightforward. We make systematical use of formulas (4.28) and (4.29). The quantities $v^{i}$ and $F_{i}^{\text {grav }}+F_{i}^{\text {press }}$ are truncated at the $1 \mathrm{PN}$ order in the first source term of $E_{i j}$, at the Newtonian order in the rest.

$$
\begin{aligned}
v^{i}= & w_{i}\left[1-\frac{1}{c^{2}}\left(\frac{1}{2} w^{2}+h_{*}+3 U_{*}\right)\right]+\frac{1}{c^{2}} A_{* i}, \\
\frac{d w_{i}}{d t}= & \partial_{i} U_{*}-\frac{\partial_{i} p_{*}}{\rho_{*}}+\frac{1}{c^{2}}\left(\frac{3}{2} w^{2}+h_{*}+2 \frac{p_{*}}{\rho_{*}}-U_{*}\right) \partial_{i} U_{*} \\
& +\frac{1}{c^{2}} \partial_{i} U_{2}-\frac{1}{c^{2}} w_{j} \partial_{i} A_{* j} \\
& -\frac{1}{c^{2}} \partial_{i}\left[\left(2 U_{*}-\gamma_{*}\left(\frac{1}{2} w^{2}+3 U_{*}\right)\right) \frac{p_{*}}{\rho_{*}}\right] .
\end{aligned}
$$

The total time derivative of the enthalpy $h_{*}$ is deduced from relation (4.29): $d h_{*} / d t=$ $\left(d \rho_{*} / d t\right)\left(\partial h_{*} / \partial p_{*}\right)\left(\partial p_{*} / \partial \rho_{*}\right)=-\gamma_{*} p_{*} \partial_{i} w_{i} / \rho_{*}+\mathcal{O}\left(1 / c^{2}\right)$. For treating the potentials $U_{*}, A_{* i}$, etc., the operator $d / d t$ is first put under its explicit form, $d / d t=\partial_{t}+v^{i} \partial_{i}$, and the partial derivative $\partial_{t}$ is next applied on the source $S$ through the integral symbol. The source variation $\partial_{t} S$ is transformed by means of the evolution equations at 
the Newtonian order. For convenience, we may redefine $U_{* i}$ as $\Delta^{-1}\left(-4 \pi G \rho_{*} w_{i}\right)$. This yields for instance

$$
\begin{aligned}
\frac{d}{d t} U_{*}= & \Delta^{-1} \partial_{t}\left(-4 \pi G \rho_{*}\right)+v^{i} \partial_{i} U_{*} \\
= & -\Delta^{-1}\left[-4 \pi G \partial_{i}\left(\rho_{*} w_{i}\right)\right]+w_{i} \partial_{i} U_{*}+\mathcal{O}\left(\frac{1}{c^{2}}\right) \\
= & -\partial_{i} U_{* i}+w_{i} \partial_{i} U_{*}+\mathcal{O}\left(\frac{1}{c^{2}}\right), \\
\frac{d}{d t} U_{* i}= & \Delta^{-1} \partial_{t}\left(-4 \pi G \rho_{*} w_{i}\right)+v^{j} \partial_{j} U_{* i} \\
= & \Delta^{-1}\left[-4 \pi G\left(-\partial_{j}\left(\rho_{*} w_{i} w_{j}\right)+\rho_{*} \partial_{i} U_{*}-\partial_{i} p_{*}\right)\right] \\
& +w_{j} \partial_{j} U_{* i}+\mathcal{O}\left(\frac{1}{c^{2}}\right) .
\end{aligned}
$$

As the potential $\partial_{t}\left(\partial_{i} \chi_{*}\right)$ occurs in $I_{i j}^{(2)}$, its time derivative must be eliminated as well. In order not to increase the number of Poisson equations, we systematically replace its old expression by the new one. It must be determined at the $2.5 \mathrm{PN}$ order, since $A_{* i}$ appears in the 1 PN part of the gravitational force (4.26).

$$
\begin{aligned}
\partial_{t} \partial_{i} \chi_{*}= & \partial_{t}\left[x^{i} \Delta^{-1}\left(-4 \pi G \rho_{*}\right)-\Delta^{-1}\left(-4 \pi G x^{i} \rho_{*}\right)\right] \\
= & -x^{i} \partial_{j} \Delta^{-1}\left(-4 \pi G \rho_{*} v^{j}\right) \\
& +\Delta^{-1}\left[-4 \pi G x^{i} \partial_{j}\left(\rho_{*} v^{j}\right)\right] \\
= & -x^{i}\left(\delta^{j k}-\left(g_{j k}^{\prime}\right)_{(5) \text { reac }}\right) \partial_{j} U_{* k} \\
& +\Delta^{-1}\left[-4 \pi G\left(\delta^{j k}-\left(g_{j k}^{\prime}\right)_{(5) \text { reac }}\right) x^{i} \partial_{j}\left(\rho_{*} w_{k}\right)\right] \\
& +\frac{1}{c^{2}}(\ldots)+\frac{1}{c^{4}}(\ldots)+\mathcal{O}\left(\frac{1}{c^{6}}\right)
\end{aligned}
$$

(the last form is obtained by using the Newtonian plus $2.5 \mathrm{PN}$ approximation for the coordinate velocity (4.24): $\left.v^{i}=w_{i}-w_{j}\left(g_{i j}^{\prime}\right)_{(5) \text { reac }}\right)$. It is sufficient to determine the total time derivative of $\partial_{t} \partial_{i} \chi_{*}$ contributing to $I_{i j}^{(2)}$ at the Newtonian order. It reads

$$
\begin{aligned}
\partial_{t}\left(\partial_{t} \partial_{i} \chi_{*}\right) & \\
=\partial_{t}\left[-x^{i} \partial_{j} U_{* j}+\Delta^{-1}\left(-4 \pi G x^{i} \partial_{j}\left(\rho_{*} w_{j}\right)\right)\right] & \\
=-x^{i} \partial_{j} \Delta^{-1}[-4 \pi & G\left(-\partial_{k}\left(\rho_{*} w_{j} w_{k}\right)\right. \\
& \left.\left.+\rho_{*} \partial_{j} U_{*}-\partial_{j} p_{*}\right)\right] \\
& +\Delta^{-1}\left[-4 \pi G x^{i}\left(-\partial_{j k}\left(\rho_{*} w_{j} w_{k}\right)\right.\right. \\
& \left.\left.+\partial_{j}\left(\rho_{*} \partial_{j} U_{*}\right)-\Delta p_{*}\right)\right] .
\end{aligned}
$$

After ruling out all temporal differentiation from $I_{i j}^{(2)}$, our result is finalized by removing, whenever possible, the space derivatives of the baryonic density $\rho_{*}$ and the pressure $p_{*}$ by integration by part. Remark that the space and time derivatives of $\gamma_{*}$ exactly cancel. All quantities $S_{i j}^{(n)}$ and $Q_{i j k}^{(n)}=(d / d t)^{n} \int d^{3} \mathbf{x} \hat{x}^{i j k} \rho_{*}$ for $n \leq 4$ are rewritten at Newtonian level following the preceding method.

Now, $S_{i j}$ and $Q_{i j k}$, as well as the hexadecapole $Q_{i j k l}=$ $\int d^{3} \mathbf{x} \hat{x}^{i j k l} \rho_{*}$, can be formally assimilated to the multipoles that parametrize the wave form $h_{i j}^{\mathrm{rad}}$ in some "radiative coordinate" grid $X^{\mu}=(T, \mathbf{X})$ up to the 3.5 order, as explained in paper [5]. After actually achieving the substitution into the wave form, the new expression for $h_{i j}^{\mathrm{rad}}(\mathbf{X}, T)$ involves one time derivation only, and is thus suitable for numerical computations. In conclusion, the present formalism allows one to build an accurate 1 $\mathrm{PN}+3.5 \mathrm{PN}$ code $(i)$ that models the fluid dynamics including the $3.5 \mathrm{PN}$ damping effect, and (ii) that permits gravitational wave extraction. All the field equations are of Poisson type, and have compact support sources. The number of time derivatives is never higher than one.

Let us list in the end the quantities to be computed and the equations to be solved for modeling the evolution of an adiabatic fluid at the $1 \mathrm{PN}+3.5 \mathrm{PN}$ approximation. For reader's convenience, we follow the presentation of Blanchet-Damour-Schäfer [5]. The notations are as close to theirs as possible, though not identical. ${ }^{3}$ It is useful to pose:

$$
\begin{aligned}
U_{5} & =\frac{c^{7}}{2}\left(g_{00}^{\prime}\right)_{(7) \text { reac }}, \\
U_{7} & =\frac{c^{9}}{2}\left(g_{00}^{\prime}\right)_{(9) \text { reac }}+3 c^{5} \Delta^{-1}\left(-4 \pi G \rho_{*} v_{(5) \text { reac }}^{i} w_{i}\right),
\end{aligned}
$$

$U_{5 i}=-\frac{c^{8}}{4}\left(g_{0 i}^{\prime}\right)_{(8) \text { reac }}$,

$h_{5 i j}=\frac{c^{5}}{2}\left(g_{i j}^{\prime}\right)_{(5) \text { reac }}$,

$h_{7 i j}=\frac{c^{7}}{2}\left(g_{i j}^{\prime}\right)_{(7) \text { reac }}-\delta^{i j} U_{5}$.

The resulting set of formulas is given below.

Thermodynamical quantities:

$T_{*}=\frac{\partial e\left(\rho_{*}, s\right)}{\partial s}$,

$\pi_{*} \equiv \frac{p\left(\rho_{*}, s\right)}{\rho_{*}}=\rho_{*} \frac{\partial e\left(\rho_{*}, s\right)}{\partial \rho_{*}}$,

$\gamma_{*} \equiv \frac{\partial \ln p\left(\rho_{*}, s\right)}{\partial \ln \rho_{*}}$,

$h_{*}=e\left(\rho_{*}, s\right)+\pi_{*}$.

Primary Poisson equations:

$\Delta U_{*}=-4 \pi G \rho_{*}$,

$\Delta U_{* i}=-4 \pi G \rho_{*} w_{i}$.

2.5 PN quantities:

$Q_{i j}^{[2]}$, see Appendix $\mathbb{B}$

$\dot{Q}_{i j}^{[2]}=\frac{d Q_{i j}^{[2]}}{d t}$

${ }^{3}$ We have the following correspondences:

\begin{tabular}{|l|c|c|c|c|}
\hline our notation & $\rho_{*}$ & $e$ & $U_{* i}$ & $A_{* i}$ \\
\hline notation of paper [5] & $r_{*}$ & $e / r_{*}$ & $U_{i}$ & $A_{i}$ \\
\hline
\end{tabular}




$$
\begin{aligned}
& h_{5 i j}=-\frac{2 G}{5} \dot{Q}_{i j}^{[2]}, \\
& \Delta R=-4 \pi G \dot{Q}_{i j}^{[2]} x^{i} \partial_{j} \rho_{*}, \\
& U_{5}=\frac{2 G}{5}\left(-\dot{Q}_{i j}^{[2]} x^{i} \partial_{j} U_{*}+R\right) .
\end{aligned}
$$

1 PN quantities with radiative corrections included:

$$
\begin{aligned}
& \alpha= 2 U_{*}-\gamma_{*}\left(\frac{1}{2} w^{2}+3 U_{*}\right) \\
&+\frac{1}{c^{5}}\left[2 U_{5}+\gamma_{*}\left(-3 U_{5}+w_{k} w_{l} h_{5 k l}\right)\right], \\
& \beta= \frac{1}{2} w^{2}+h_{*}+3 U_{*}, \\
& \delta= \frac{3}{2} w^{2}+h_{*}+2 \pi_{*}-U_{*}, \\
& \zeta_{i}=-\partial_{j}\left(\rho_{*} w_{i} w_{j}\right)+\rho_{*} \partial_{i} U_{*}-\partial_{i} p_{*}, \\
& \Delta U_{2}=-4 \pi G \rho_{*} \delta, \\
& \Delta D_{i}=-4 \pi G \zeta_{i}, \\
& \Delta E_{i}=-4 \pi G x^{i} \partial_{j} \zeta_{j}, \\
& \Delta C_{i}=-4 \pi G\left(\delta^{j k}-\frac{2}{c^{5}} h_{5 j k}\right) x^{i} \partial_{j}\left(\rho_{*} w_{k}\right), \\
& A_{* i}=4\left(\delta^{i k}-\frac{2}{c^{5}} h_{5 i k}\right) U_{* k}+\frac{1}{2} C_{i} \\
& \quad-\frac{1}{2} x^{i}\left(\delta^{j k}-\frac{2}{c^{5}} h_{5 j k}\right) \partial_{j} U_{* k} .
\end{aligned}
$$

3.5 PN quantities:

$I_{2 i j}^{[2]}, Q_{i j}^{[3]}, Q_{i j}^{[4]}, Q_{i j k}^{[4]}, S_{i j}^{[3]}$, see Appendix $\mathbb{B}$

$$
\begin{aligned}
& \dot{I}_{2 i j}^{[2]}=\frac{d I_{2}^{[2]}}{d t}, \\
& \dot{Q}_{i j}^{[4]}=\frac{d Q_{i j}^{[4]}}{d t}, \\
& \dot{Q}_{i j k}^{[4]}=\frac{d Q_{i j k}^{[4]}}{d t}, \\
& \dot{S}_{i j}^{[4]}=\frac{d S_{i j}^{[4]}}{d t},
\end{aligned}
$$$$
\Delta R_{2}=-4 \pi G\left[\dot{I}_{2 k l}^{[2]} x^{k} \partial_{l} \rho_{*}+Q_{k l}^{[3]} x^{k}\left(\rho_{*} \partial_{l} U_{*}+\partial_{l}\left(\rho_{*} \delta\right)\right)\right.
$$$$
+3 \rho_{*} w_{k} w_{l} Q_{k l}^{[3]}-\frac{5}{126} \dot{Q}_{k l m}^{[4]} x^{k} x^{l} \partial_{m} \rho_{*}
$$$$
+Q_{k l}^{[4]} x^{k}\left(\frac{1}{2} x^{l} \partial_{t} \rho_{*}-4 \rho_{*} w_{l}\right)
$$$$
+\dot{Q}_{k l}^{[4]} x^{k}\left(-\frac{17}{42} x^{l} x^{m} \partial_{m} \rho_{*}+\frac{11}{42} r^{2} \partial_{l} \rho_{*}-\rho_{*} x^{l}\right)
$$$$
\left.-\frac{8}{9} \epsilon_{k l m} \dot{S}_{m n}^{[3]} x^{l} x^{n} \partial_{k} \rho_{*}-\rho_{*} R\right] \text {, }
$$

$\Delta R_{i}=-4 \pi G\left[Q_{k l}^{[3]} x^{k} \partial_{l}\left(\rho_{*} w_{i}\right)-\rho_{*} w_{k} Q_{i k}^{[3]}-\rho_{*} x^{k} Q_{i k}^{[4]}\right.$

$$
\begin{aligned}
& \left.-\frac{1}{8} \partial_{t}\left(Q_{k l}^{[3]} x^{i} x^{k} \partial_{l} \rho_{*}\right)\right], \\
U_{7}= & \frac{2 G}{5}\left[-\dot{I}_{2 k l}^{[2]} x^{k} \partial_{l} U_{*}+Q_{k l}^{[3]} x^{k}\left(-\partial_{l} U_{2}+2 U_{*} \partial_{l} U_{*}\right)\right. \\
+ & Q_{k l}^{[4]} x^{k}\left(-\frac{1}{2} x^{l} \partial_{t} U_{*}+A_{* l}\right)+\frac{5}{126} \dot{Q}_{k l m}^{[4]} x^{k} x^{l} \partial_{m} U_{*} \\
+ & \dot{Q}_{k l}^{[4]} x^{k}\left(\frac{17}{42} x^{l} x^{m} \partial_{m} U_{*}-\frac{11}{42} r^{2} \partial_{l} U_{*}\right) \\
& \left.-\frac{8}{9} \epsilon_{k l m} \dot{S}_{m n}^{[3]} x^{l} x^{n} \partial_{k} U_{*}-2 U_{*} R+R_{2}\right], \\
U_{5 i}= & \frac{2 G}{5}\left[-\frac{1}{4} Q_{k l}^{[3]} x^{k} \partial_{l} A_{* i}-\frac{1}{4} Q_{i k}^{[3]} A_{* k}+Q_{i k}^{[4]} x^{k} U_{*}\right. \\
& \left.+\frac{1}{8} x^{i} \partial_{t} R+R_{i}\right], \\
= & \frac{2 G}{5}\left[-2 \dot{I}_{2 i j}^{[2]}-2 Q_{i j}^{[3]} U_{*}+\frac{5}{63} \dot{Q}_{i j k}^{[4]} x^{k}+\frac{2}{7} x^{k} x^{(i} \dot{Q}_{j) k}^{[4]}\right. \\
h_{7 i j} & \left.-\frac{11}{42} \dot{Q}_{i j}^{[4]} r^{2}-\frac{8}{9} \epsilon_{k l(i)} \dot{S}_{j) l}^{[3]} x^{k}-\frac{2}{21} \delta^{i j} x^{k} x^{l} \dot{Q}_{k l}^{[4]}\right] .
\end{aligned}
$$

Velocity and forces:

$$
\begin{aligned}
v^{i}=w_{i} & +\frac{1}{c^{2}}\left(-\beta w_{i}+A_{* i}\right)-\frac{2}{c^{5}} w_{k} h_{5 i k} \\
- & \frac{1}{c^{7}}\left(-4 U_{5 i}+2 w_{k} h_{7 i k}+w_{i}\left(3 U_{5}-w_{k} w_{l} h_{5 k l}\right)\right. \\
- & \left.2\left(\beta+2 U_{*}\right) w_{k} h_{5 i k}+2 A_{* k} h_{5 i k}\right) \\
F_{i}^{\text {press }}= & -\left(1+\frac{\alpha}{c^{2}}\right)\left(\partial_{i} h_{*}-T_{*} \partial_{i} s\right)-\frac{1}{c^{2}} \pi_{*} \partial_{i} \alpha, \\
F_{i}^{\text {grav }}= & \left(1+\frac{\delta}{c^{2}}\right) \partial_{i} U_{*}+\frac{1}{c^{2}} \partial_{i} U_{2}-\frac{1}{c^{2}} w^{j} \partial_{i} A_{* j}+\frac{1}{c^{5}} \partial_{i} U_{5} \\
& +\frac{1}{c^{7}}\left(\partial_{i} U_{7}-4 w_{k} \partial_{i} U_{5 k}+w_{k} w_{l} \partial_{i} h_{7 k l}\right. \\
& +\left(U_{5}-5 w_{k} w_{l} h_{5 k l}\right) \partial_{i} U_{*}+\left(\delta+2 U_{*}\right) \partial_{i} U_{5} \\
& \left.+2 h_{5 k l} w_{k} \partial_{i} A_{* l}\right) \cdot
\end{aligned}
$$

Evolution system:

$$
\begin{aligned}
& \partial_{t} \rho_{*}=-\partial_{i}\left(\rho_{*} v^{i}\right), \\
& \partial_{t} s=-v^{i} \partial_{i} s, \\
& \partial_{t} w_{i}=-v^{j} \partial_{j} w_{i}+F_{i}^{\text {grav }}+F_{i}^{\text {press }} . \\
& \text { Gravitational wave form }\left(R=\sqrt{X^{i} X^{i}}, \mathbf{N}=\mathbf{X} / R\right) \text { : } \\
& h_{i j}^{\text {rad }}(\mathbf{X}, T)=\frac{2 G}{c^{4} R} P_{i j k l}(\mathbf{N})\left\{I_{k l}^{(2)}+\frac{1}{3 c} N_{a} Q_{a k l}^{[3]}\right. \\
& \quad+\frac{4}{3 c} \epsilon_{a b(k} S_{l) a}^{[2]} N_{b}+\frac{1}{12 c^{2}} N_{a} N_{b} \dot{Q}_{a b k l}^{[3]} \\
& \left.\quad+\frac{1}{2 c^{2}} \epsilon_{a b(k} \dot{S}_{l) a c}^{[2]} N_{b} N_{c}\right\} .
\end{aligned}
$$




\section{Acknowledgment}

This work was supported by the EU Program "Improving the Human Research Potential and the SocioEconomic Knowledge Base" (Research Training Network Contract HPRN-CT-2000-00137). We thank M. Brügmann for checks of several formulas.

\section{APPENDIX A: SET OF FORMULAS IN THE $\left\{\tilde{\rho}, \mathbf{w}_{\mathbf{i}}, \mathbf{s}\right\}$ REPRESENTATION}

We give here the set of equations describing the $1+$ $3.5 \mathrm{PN}$ dynamics by means of the matter variables $\tilde{\rho}=$ $\rho_{*}+\sigma_{(7)}, w_{i}$, and $s$ (see section [V).

Thermodynamical quantities:

$$
\begin{aligned}
\tilde{T} & =\frac{\partial e(\tilde{\rho}, s)}{\partial s} \\
\tilde{\pi} & \equiv \frac{p(\tilde{\rho}, s)}{\tilde{\rho}}=\tilde{\rho} \frac{\partial e(\tilde{\rho}, s)}{\partial \tilde{\rho}} \\
\tilde{\gamma} & \equiv \frac{\partial \ln p(\tilde{\rho}, s)}{\partial \ln \tilde{\rho}} \\
\tilde{h} & =e(\tilde{\rho}, s)+\tilde{\pi} .
\end{aligned}
$$

Primary Poisson equations:

$\Delta \tilde{U}=-4 \pi G \tilde{\rho}$,

$\Delta \tilde{U}_{i}=-4 \pi G \tilde{\rho} w_{i}$.

2.5 PN quantities:

$Q_{i j}^{[2]}$, see Appendix [B] with the substitutions $\rho_{*} \rightarrow \tilde{\rho}$, $U_{*} \rightarrow \tilde{U}$, etc...;

$$
\begin{aligned}
& \dot{Q}_{i j}^{[2]}=\frac{d Q_{i j}^{[2]}}{d t} \\
& h_{5 i j}=-\frac{2 G}{5} \dot{Q}_{i j}^{[2]}, \\
& \Delta R=-4 \pi G \dot{Q}_{i j}^{[2]} x^{i} \partial_{j} \tilde{\rho}, \\
& U_{5}=\frac{2 G}{5}\left(-\dot{Q}_{i j}^{[2]} x^{i} \partial_{j} \tilde{U}+R\right) .
\end{aligned}
$$

$1 \mathrm{PN}$ quantities with radiative corrections included:

$$
\begin{aligned}
& \alpha=2 \tilde{U}-\tilde{\gamma}\left(\frac{1}{2} w^{2}+3 \tilde{U}\right)+\frac{2}{c^{5}} U_{5}(1-\tilde{\gamma}) \\
& \beta=\frac{1}{2} w^{2}+\tilde{h}+3 \tilde{U} \\
& \delta=\frac{3}{2} w^{2}+\tilde{h}+2 \tilde{\pi}-\tilde{U} \\
& \zeta_{i}=-\partial_{j}\left(\tilde{\rho} w_{i} w_{j}\right)+\tilde{\rho} \partial_{i} \tilde{U}-\partial_{i} \tilde{p} \\
& \Delta U_{2}=-4 \pi G \tilde{\rho} \delta \\
& \Delta D_{i}=-4 \pi G \zeta_{i} \\
& \Delta E_{i}=-4 \pi G x^{i} \partial_{j} \zeta_{j}
\end{aligned}
$$

$$
\begin{aligned}
\Delta C_{i}= & -4 \pi G\left(\delta^{j k}-\frac{2}{c^{5}} h_{5 j k}\right) x^{i} \partial_{j}\left(\tilde{\rho} w_{k}\right) \\
\tilde{A}_{i}= & 4\left(\delta^{i k}-\frac{2}{c^{5}} h_{5 i k}\right) \tilde{U}_{k}+\frac{1}{2} C_{i} \\
& -\frac{1}{2} x^{i}\left(\delta^{j k}-\frac{2}{c^{5}} h_{5 j k}\right) \partial_{j} \tilde{U}_{k}
\end{aligned}
$$

3.5 PN quantities:

$I_{2}{ }_{i j}^{[2]}, Q_{i j}^{[3]}, Q_{i j}^{[4]}, Q_{i j k}^{[4]}, S_{i j}^{[3]}$, see Appendix [B] with the substitutions $\rho_{*} \rightarrow \tilde{\rho}, U_{*} \rightarrow \tilde{U}$, etc...;

$$
\begin{aligned}
& \dot{I}_{2 i j}^{[2]}=\frac{d I_{2}^{[2]}}{d t} \\
& \dot{Q}_{i j}^{[4]}=\frac{d Q_{i j}^{[4]}}{d t}, \\
& \dot{Q}_{i j k}^{[4]}=\frac{d Q_{i j k}^{[4]}}{d t}, \\
& \dot{S}_{i j}^{[4]}=\frac{d S_{i j}^{[4]}}{d t}, \\
& \theta=\frac{2 G}{5}\left[Q_{i j}^{[3]}\left(x^{i} \partial_{j} \tilde{U}-w_{i} w_{j}\right)-R\right] \text {, } \\
& \Delta R_{2}=-4 \pi G\left[\dot{I}_{2 k l}^{[2]} x^{k} \partial_{l} \tilde{\rho}+Q_{k l}^{[3]} x^{k} \partial_{l}(\tilde{\rho} \delta)\right. \\
& +4 \tilde{\rho} w_{k} w_{l} Q_{k l}^{[3]}-\frac{5}{126} \dot{Q}_{k l m}^{[4]} x^{k} x^{l} \partial_{m} \tilde{\rho} \\
& +Q_{k l}^{[4]} x^{k}\left(\frac{1}{2} x^{l} \partial_{t} \tilde{\rho}-4 \tilde{\rho} w_{l}\right) \\
& +\dot{Q}_{k l}^{[4]} x^{k}\left(-\frac{17}{42} x^{l} x^{m} \partial_{m} \tilde{\rho}+\frac{11}{42} r^{2} \partial_{l} \tilde{\rho}-\tilde{\rho} x^{l}\right) \\
& \left.-\frac{8}{9} \epsilon_{k l m} \dot{S}_{m n}^{[3]} x^{l} x^{n} \partial_{k} \tilde{\rho}\right] \text {, } \\
& \Delta R_{i}=-4 \pi G\left[Q_{k l}^{[3]} x^{k} \partial_{l}\left(\tilde{\rho} w_{i}\right)-\tilde{\rho} w_{k} Q_{i k}^{[3]}-\tilde{\rho} x^{k} Q_{i k}^{[4]}\right. \\
& \left.-\frac{1}{8} \partial_{t}\left(Q_{k l}^{[3]} x^{i} x^{k} \partial_{l} \tilde{\rho}\right)\right] \\
& U_{7}=\frac{2 G}{5}\left[-\dot{I}_{2 k l}^{[2]} x^{k} \partial_{l} \tilde{U}+Q_{k l}^{[3]} x^{k}\left(-\partial_{l} U_{2}+2 \tilde{U} \partial_{l} \tilde{U}\right)\right. \\
& +Q_{k l}^{[4]} x^{k}\left(-\frac{1}{2} x^{l} \partial_{t} \tilde{U}+\tilde{A}_{l}\right) \\
& +\frac{5}{126} \dot{Q}_{k l m}^{[4]} x^{k} x^{l} \partial_{m} \tilde{U} \\
& +\dot{Q}_{k l}^{[4]} x^{k}\left(\frac{17}{42} x^{l} x^{m} \partial_{m} \tilde{U}-\frac{11}{42} r^{2} \partial_{l} \tilde{U}\right) \\
& \left.-\frac{8}{9} \epsilon_{k l m} \dot{S}_{m n}^{[3]} x^{l} x^{n} \partial_{k} \tilde{U}-2 \tilde{U} R+R_{2}\right] \text {, } \\
& U_{5 i}=\frac{2 G}{5}\left[-\frac{1}{4} Q_{k l}^{[3]} x^{k} \partial_{l} \tilde{A}_{i}-\frac{1}{4} Q_{i k}^{[3]} \tilde{A}_{k}+Q_{i k}^{[4]} x^{k} \tilde{U}\right. \\
& \left.+\frac{1}{8} x^{i} \partial_{t} R+R_{i}\right] \text {, }
\end{aligned}
$$




$$
\begin{aligned}
h_{7 i j}= & \frac{2 G}{5}\left[-2 \dot{I}_{2 i j}^{[2]}-2 Q_{i j}^{[3]} \tilde{U}+\frac{5}{63} \dot{Q}_{i j k}^{[4]} x^{k}+\frac{2}{7} x^{k} x^{(i} \dot{Q}_{j) k}^{[4]}\right. \\
& \left.-\frac{11}{42} \dot{Q}_{i j}^{[4]} r^{2}-\frac{8}{9} \epsilon_{k l(i} \dot{S}_{j) l}^{[3]} x^{k}-\frac{2}{21} \delta^{i j} x^{k} x^{l} \dot{Q}_{k l}^{[4]}\right] .
\end{aligned}
$$

Baryonic density, velocity and forces:

$$
\begin{aligned}
\rho_{*}= & \tilde{\rho}\left(1-\frac{\theta}{c^{7}}\right) \\
v^{i}= & w_{i}+\frac{1}{c^{2}}\left(-\beta w_{i}+\tilde{A}_{i}\right)-\frac{2}{c^{5}} w_{k} h_{5 i k} \\
& -\frac{1}{c^{7}}\left(-4 U_{5 i}+2 w_{k} h_{7 i k}+w_{i}\left(3 U_{5}-w_{k} w_{l} h_{5 k l}\right)\right. \\
& \left.-2(\beta+2 \tilde{U}) w_{k} h_{5 i k}+2 \tilde{A}_{k} h_{5 i k}\right), \\
F_{i}^{\text {press }} & =-\left[1+\frac{\alpha}{c^{2}}+\frac{\theta}{c^{7}}\right]\left(\partial_{i} \tilde{h}-\tilde{T} \partial_{i} s\right)-\frac{1}{c^{2}} \tilde{\pi} \partial_{i} \alpha
\end{aligned}
$$

$$
\begin{aligned}
F_{i}^{\text {grav }}= & \left(1+\frac{\delta}{c^{2}}\right) \partial_{i} \tilde{U}+\frac{1}{c^{2}} \partial_{i} U_{2}-\frac{1}{c^{2}} w^{j} \partial_{i} \tilde{A}_{j}+\frac{1}{c^{5}} \partial_{i} U_{5} \\
& +\frac{1}{c^{7}}\left(\partial_{i} U_{7}-4 w_{k} \partial_{i} U_{5 k}+w_{k} w_{l} \partial_{i} h_{7 k l}\right. \\
& +\left(U_{5}-5 w_{k} w_{l} h_{5 k l}\right) \partial_{i} \tilde{U}+(\delta+2 \tilde{U}) \partial_{i} U_{5} \\
& \left.+2 h_{5 k l} w_{k} \partial_{i} \tilde{A}_{l}\right) .
\end{aligned}
$$

Evolution system:

$$
\begin{aligned}
& \partial_{t} \rho_{*}=-\partial_{i}\left(\rho_{*} v^{i}\right), \\
& \partial_{t} s=-v^{i} \partial_{i} s \\
& \partial_{t} w_{i}=-v^{j} \partial_{j} w_{i}+F_{i}^{\mathrm{grav}}+F_{i}^{\mathrm{press}} .
\end{aligned}
$$

(A32)

\section{APPENDIX B: TIME DERIVATIVES OF RELEVANT MULTIPOLE MOMENTS}

This Appendix presents the full explicit expressions for the time derivatives of the multipole moments used in the present formalism.

$$
\begin{aligned}
& I_{i j}^{(1)}=Q_{i j}^{[1]}+\frac{1}{c^{2}} I_{2}^{[1]}+\mathcal{O}\left(\frac{1}{c^{4}}\right) \\
& =\operatorname{STF} \int\left\{2 \rho_{*} x^{i} w_{j}+\frac{1}{c^{2}}\left[\rho_{*}\left(2 A_{* i} x^{j}+\frac{11}{7} r^{2} w_{j} \partial_{i} U_{*}+\frac{10}{21} x^{p} w_{i} w_{j} w_{p}\right)+\frac{22}{21} p_{*} r^{2} \partial_{i} w_{j}\right.\right. \\
& +\rho_{*} x^{i}\left[\frac{11}{21} r^{2} w_{p} \partial_{j p} U_{*}-\frac{11}{21} r^{2} \partial_{j p} U_{* p}-8 U_{*} w_{j}-\frac{4}{21} w_{j} w^{2}\right] \\
& +x^{i} x^{p}\left[\rho_{*}\left(-\frac{46}{21} w_{j} \partial_{p} U_{*}+\frac{10}{21} w_{p} \partial_{j} U_{*}\right)-\frac{4}{7} p_{*}\left(\partial_{p} w_{j}+\partial_{j} w_{p}\right)\right]+x^{i} x^{j}\left[\rho_{*}\left(\partial_{p} U_{* p}-\frac{3}{7} w_{p} \partial_{p} U_{*}\right)+\frac{8}{21} p_{*} \partial_{p} w_{p}\right] \\
& \left.\left.+\frac{17}{21} \rho_{*} x^{i} x^{j} x^{p}\left[\partial_{p q} U_{* q}-w_{q} \partial_{p q} U_{*}\right]\right]\right\} d^{3} \mathbf{x}+\mathcal{O}\left(\frac{1}{c^{4}}\right) \\
& I_{i j}^{(2)}=Q_{i j}^{[2]}+\frac{1}{c^{2}} I_{2 i j}^{[2]}+\mathcal{O}\left(\frac{1}{c^{4}}\right)=\operatorname{STF} \int\left\{2 \rho_{*} x^{i} \partial_{j} U_{*}+2 \rho_{*} w_{i} w_{j}\right. \\
& +\frac{1}{c^{2}}\left[\rho _ { * } \left(8 D_{i} x^{j}+E_{i} x^{j}+\frac{11}{7} r^{2} \partial_{i} U_{*} \partial_{j} U_{*}+4 A_{* i} w_{j}-\frac{44}{21} r^{2} w_{j} \partial_{i p} U_{* p}-14 U_{*} w_{i} w_{j}\right.\right. \\
& \left.+\frac{44}{21} r^{2} w_{j} w_{p} \partial_{i p} U_{*}-\frac{5}{7} w_{i} w_{j} w^{2}\right)+p_{*}\left(-\frac{22}{21} r^{2} \partial_{i} w_{p} \partial_{p} w_{j}+\frac{22}{21}\left(1-\gamma_{*}\right) r^{2} \partial_{i} w_{j} \partial_{p} w_{p}+\frac{22}{7} r^{2} \partial_{i j} U_{*}\right) \\
& -2 h_{*} \rho_{*} w_{i} w_{j}+2 p_{*} w_{i} w_{j}+\frac{22}{21} r^{2} \partial_{i} h_{*} \partial_{j} p_{*}-\frac{22}{21} r^{2} T_{*} \partial_{i} s \partial_{j} p_{*} \\
& +x^{p}\left[\rho_{*}\left(-\frac{12}{7} w_{i} w_{j} \partial_{p} U_{*}+\frac{32}{7} w_{j} w_{p} \partial_{i} U_{*}\right)+p_{*}\left(\frac{8}{21} w_{i} \partial_{p} w_{j}+\frac{8}{21} w_{j} \partial_{i} w_{p}+\frac{64}{21} w_{p} \partial_{i} w_{j}\right)\right] \\
& +x^{i}\left[\rho_{*}\left(2 \partial_{j} U_{2}-10 U_{*} \partial_{j} U_{*}+10 w_{j} \partial_{p} U_{* p}-2 w_{p} \partial_{j} A_{* p}+2 w_{p} \partial_{p} A_{* j}+\frac{23}{7} w^{2} \partial_{j} U_{*}-\frac{80}{7} w_{j} w_{p} \partial_{p} U_{*}\right)\right. \\
& \left.+p_{*}\left(-2 \partial_{j} U_{*}+\frac{8}{21} w_{j} \partial_{p} w_{p}-\frac{20}{21} w_{p} \partial_{j} w_{p}-\frac{20}{21} w_{p} \partial_{p} w_{j}\right)+2 h_{*} \rho_{*} \partial_{j} U_{*}\right] \\
& +x^{i} x^{p}\left[\rho_{*}\left(-\frac{12}{7} \partial_{j} U_{*} \partial_{p} U_{*}+\frac{80}{21} w_{j} \partial_{p q} U_{* q}-\frac{32}{21} w_{p} \partial_{j q} U_{* q}-\frac{80}{21} w_{j} w_{q} \partial_{p q} U_{*}+\frac{32}{21} w_{p} w_{q} \partial_{j q} U_{*}\right)\right. \\
& +p_{*}\left(\frac{4}{7} \partial_{p} w_{q} \partial_{q} w_{j}+\frac{4}{7} \partial_{j} w_{q} \partial_{q} w_{p}-\frac{4}{7}\left(1-\gamma_{*}\right) \partial_{p} w_{j} \partial_{q} w_{q}-\frac{4}{7}\left(1-\gamma_{*}\right) \partial_{j} w_{p} \partial_{q} w_{q}-\frac{24}{7} \partial_{j p} U_{*}\right) \\
& \left.-\frac{8}{7} \partial_{j} p_{*} \partial_{p} h_{*}+\frac{8}{7} T_{*} \partial_{j} p_{*} \partial_{p} s\right]
\end{aligned}
$$




$$
\begin{aligned}
& +x^{i} x^{j}\left[\rho_{*}\left(-\frac{3}{7} \partial_{p} U_{*} \partial_{p} U_{*}+\frac{47}{21} w_{q} \partial_{p q} U_{* p}-\frac{26}{21} w_{p} w_{q} \partial_{p q} U_{*}\right)\right. \\
& +p_{*}\left(\frac{8}{21}\left(1-\gamma_{*}\right)\left(\partial_{p} w_{p}\right)^{2}-\frac{8}{21} \partial_{q} w_{p} \partial_{p} w_{q}-\frac{6}{7}\left(-4 \pi G \rho_{*}\right)+\frac{8}{21} \partial_{p} h_{*} \partial_{p} p_{*}-\frac{8}{21} T_{*} \partial_{p} s \partial_{p} p_{*}\right] \\
& +r^{2} x^{i}\left[\rho_{*}\left(\frac{11}{21} \partial_{p} U_{*} \partial_{j p} U_{*}-\frac{11}{21} \partial_{j p} D_{p}-\frac{22}{21} w_{q} \partial_{j p q} U_{* p}+\frac{11}{21} w_{p} w_{q} \partial_{j p q} U_{*}\right)+\frac{11}{21} p_{*} \partial_{j}\left(-4 \pi G \rho_{*}\right)\right] \\
& +x^{i} x^{j} x^{p}\left[\rho _ { * } \left(-\frac{17}{21} \partial_{q} U_{*} \partial_{p q} U_{*}+\frac{17}{21} \partial_{p q} D_{q}+\frac{34}{21} w_{r} \partial_{p q r} U_{* q}\right.\right. \\
& \left.\left.\left.\left.-\frac{17}{21} w_{q} w_{r} \partial_{p q r} U_{*}\right)-\frac{17}{21} p_{*} \partial_{p}\left(-4 \pi G \rho_{*}\right)\right]\right]\right\} d^{3} \mathbf{x}+\mathcal{O}\left(\frac{1}{c^{4}}\right), \\
& Q_{i j}^{[3]}=\operatorname{STF} \int\left\{4 p_{*} \partial_{i} w_{j}+6 \rho_{*} w_{j} \partial_{i} U_{*}+2 \rho_{*} x^{i}\left[w_{p} \partial_{j p} U_{*}-\partial_{j p} U_{* p}\right]\right\} d^{3} \mathbf{x}, \\
& Q_{i j}^{[4]}=\operatorname{STF} \int\left\{4 \rho_{*}\left(\partial_{i} h_{*}-T_{*} \partial_{i} s\right)\left(\partial_{j} h_{*}-T_{*} \partial_{j} s\right)+2 \rho_{*}\left(3 \partial_{i} U_{*} \partial_{j} U_{*}-4 w_{j} \partial_{i p} U_{* p}+4 w_{j} w_{p} \partial_{i p} U_{*}\right)\right. \\
& +4 p_{*}\left(-\partial_{i} w_{p} \partial_{p} w_{j}+\partial_{i} w_{j} \partial_{p} w_{p}-\gamma_{*} \partial_{i} w_{j} \partial_{p} w_{p}+3 \partial_{i j} U_{*}\right. \\
& \left.+x^{i}\left[2 \rho_{*}\left(\partial_{j p} U_{*} \partial_{p} U_{*}-\partial_{j p} D_{p}-2 w_{q} \partial_{j p q} U_{* p}+w_{p} w_{q} \partial_{j p q} U_{*}\right)+2 p_{*} \partial_{j}\left(-4 \pi G \rho_{*}\right)\right]\right\} d^{3} \mathbf{x}, \\
& Q_{i j k}^{[1]}=\operatorname{STF} \int\left\{3 \rho_{*} x^{i} x^{j} w_{k}\right\} d^{3} \mathbf{x}, \\
& Q_{i j k}^{[2]}=\operatorname{STF} \int\left\{3 \rho_{*} x^{i} x^{j} \partial_{k} U_{*}+6 \rho_{*} x^{i} w_{j} w_{k}\right\} d^{3} \mathbf{x}, \\
& Q_{i j k}^{[3]}=\operatorname{STF} \int\left\{6 \rho_{*} w_{i} w_{j} w_{k}+x^{i}\left[18 \rho_{*} w_{k} \partial_{j} U_{*}+12 p_{*} \partial_{j} w_{k}\right]+3 \rho_{*} x^{i} x^{j}\left[-\partial_{k p} U_{* p}+w_{p} \partial_{k p} U_{*}\right]\right\} d^{3} \mathbf{x}, \\
& Q_{i j k}^{[4]}=\operatorname{STF} \int\left\{36 \rho_{*} w_{j} w_{k} \partial_{i} U_{*}+48 p_{*} w_{j} \partial_{i} w_{k}+x^{i}\left[12 \rho_{*}\left(\partial_{j} h_{*}-T_{*} \partial_{j} s\right)\left(\partial_{k} h_{*}-T_{*} \partial_{k} s\right)\right.\right. \\
& +\rho_{*}\left(18 \partial_{j} U_{*} \partial_{k} U_{*}-24 w_{k} \partial_{j p} U_{* p}+24 w_{k} w_{p} \partial_{j p} U_{*}\right) \\
& \left.+p_{*}\left(-12 \partial_{j} w_{p} \partial_{p} w_{k}+12 \partial_{j} w_{k} \partial_{p} w_{p}-12 \gamma_{*} \partial_{j} w_{k} \partial_{p} w_{p}+36 \partial_{j k} U_{*}\right)\right] \\
& \left.+x^{i} x^{j}\left[\rho_{*}\left(3 \partial_{p} U_{*} \partial_{k p} U_{*}-3 \partial_{k p} D_{p}-6 w_{q} \partial_{k p q} U_{* p}+3 w_{p} w_{q} \partial_{k p q} U_{*}\right)\right]+3 p_{*} \partial_{k}\left(-4 \pi G \rho_{*}\right)\right\} d^{3} \mathbf{x}, \\
& Q_{i j k l}^{[1]}=\operatorname{STF} \int\left\{4 \rho_{*} x^{i} x^{j} x^{k} w_{l}\right\} d^{3} \mathbf{x}, \\
& Q_{i j k l}^{[2]}=\operatorname{STF} \int\left\{4 \rho_{*} x^{i} x^{j} x^{k} \partial_{l} U_{*}+12 \rho_{*} x^{i} x^{j} w_{k} w_{l}\right\} d^{3} \mathbf{x}, \\
& Q_{i j k l}^{[3]}=\operatorname{STF} \int\left\{24 \rho_{*} x^{i} w_{j} w_{k} w_{l}+x^{i} x^{j}\left(36 \rho_{*} w_{l} \partial_{k} U_{*}+24 p_{*} \partial_{k} w_{l}\right)+4 \rho_{*} x^{i} x^{j} x^{k}\left(-\partial_{l p} U_{* p}+w_{p} \partial_{l p} U_{*}\right)\right\} d^{3} \mathbf{x}, \\
& S_{i j}^{[1]}=\operatorname{STF} \int \epsilon_{p q i}\left\{\rho_{*} x^{j} x^{p} \partial_{q} U_{*}+\rho_{*} x^{p} w_{j} w_{q}\right\} d^{3} \mathbf{x}, \\
& S_{i j}^{[2]}=\mathrm{STF} \int \epsilon_{p q i}\left\{x^{p}\left[\rho_{*} w_{q} \partial_{j} U_{*}+p_{*} \partial_{j} w_{q}\right]\right. \\
& \left.+\left[x^{p}\left(2 \rho_{*} w_{j} \partial_{q} U_{*}+p_{*} \partial_{q} w_{j}\right)+x^{j} \rho_{*} w_{p} \partial_{q} U_{*}+\rho_{*} x^{j} x^{p}\left(w_{r} \partial_{q r} U_{*}-\partial_{q r} U_{* r}\right)\right]\right\} d^{3} \mathbf{x}, \\
& S_{i j}^{[3]}=\operatorname{STF} \int \epsilon_{p q i}\left\{\left[-p_{*} w_{q} \partial_{j} w_{p}+\rho_{*} x^{p}\left[-w_{q} \partial_{j r} U_{* r}+w_{q} w_{r} \partial_{j r} U_{*}\right]\right.\right. \\
& \left.+p_{*} x^{p}\left[-\partial_{j} w_{r} \partial_{r} w_{q}+\partial_{j} w_{q} \partial_{r} w_{r}-\gamma_{*} \partial_{j} w_{q} \partial_{r} w_{r}\right]\right] \\
& +\left[2 \rho_{*} x^{p}\left(\partial_{j} h_{*}-T_{*} \partial_{j} s\right)\left(\partial_{q} h_{*}-T_{*} \partial_{q} s\right)+p_{*} w_{p} \partial_{q} w_{j}+3 \rho_{*} w_{j} w_{p} \partial_{q} U_{*}\right. \\
& +3 \rho_{*} x^{p}\left[\partial_{j} U_{*} \partial_{q} U_{*}-w_{j} \partial_{q r} U_{* r}+w_{j} w_{r} \partial_{q r} U_{*}\right]+p_{*} x^{p}\left[\partial_{q} w_{j} \partial_{r} w_{r}-\partial_{r} w_{j} \partial_{q} w_{r}-\gamma_{*} \partial_{q} w_{j} \partial_{r} w_{r}+6 \partial_{j q} U_{*}\right] \\
& +2 \rho_{*} x^{j}\left[w_{p} w_{r} \partial_{q r} U_{*}-w_{p} \partial_{q r} U_{* r}\right] \\
& \left.\left.+x^{j} x^{p}\left[\rho_{*}\left(\partial_{q r} U_{*} \partial_{r} U_{*}-\partial_{q r} D_{r}-2 w_{s} \partial_{q r s} U_{* r}+w_{r} w_{s} \partial_{q r s} U_{*}\right)+p_{*} \partial_{q}\left(-4 \pi G \rho_{*}\right)\right]\right]\right\} d^{3} \mathbf{x}, \\
& S_{i j k}^{[1]}=\operatorname{STF} \int \epsilon_{p q i}\left\{\rho_{*} x^{j} x^{k} x^{p} \partial_{q} U_{*}+2 \rho_{*} x^{j} x^{p} w_{k} w_{q}\right\} d^{3} \mathbf{x},
\end{aligned}
$$




$$
\begin{aligned}
S_{i j k}^{[2]}= & \operatorname{STF} \int \epsilon_{p q i}\left\{2 \rho_{*} x^{p} w_{j} w_{k} w_{q}+\rho_{*} x^{j} x^{k} w_{p} \partial_{q} U_{*}+x^{j} x^{p}\left[2 \rho_{*}\left(2 w_{k} \partial_{q} U_{*}+w_{q} \partial_{k} U_{*}\right)+2 p_{*}\left(\partial_{q} w_{k}+\partial_{k} w_{q}\right)\right]\right. \\
& \left.-\rho_{*} x^{j} x^{k} x^{p}\left[\partial_{q r} U_{* r}-w_{r} \partial_{q r} U_{*}\right]\right\} d^{3} \mathbf{x} .
\end{aligned}
$$

[1] L. Blanchet, Phys. Rev. D 55, 714 (1997).

[2] G. Schäfer, Lett. Nuovo Cim. 36, 105 (1983).

[3] T. Damour and G. Schäfer, Gen. Rel. Grav. 17, 879 (1985).

[4] L. Rezzolla, M. Shibata, H. Asada, T. W. Baumgarte, and S. L. Shapiro, Astrophys. J. 525, 935 (1999).

[5] L. Blanchet, T. Damour, and G. Schäfer, Mon. Not. R. astr. Soc. 242, 289 (1990).

[6] K. Oohara and T. Nakamura, in Relativistic Gravitation and Gravitational Radiation, edited by J.-A. Marck and J.-P. Lasota, Les Houches (Cambridge University Press, 1997).

[7] D. I. Jones, N. Andersson, and E. Gourgoulhon, in preparation.

[8] N. Stergioulas, Living Reviews 1, 1998-8 (1998).

[9] S. Chandrasekhar, Phys. Rev. Lett. 24, 611 (1970).
[10] J. Friedman and B. Schutz, Astrophys. J. 222, 281 (1978).

[11] H. Dimmelmeier, J. A. Font, and E. Müller, Astron. Astrophys. 388, 917 (2002).

[12] M. Shibata, Phys. Rev. D 67, 024033 (2003).

[13] L. Schwartz, Analyse (Hermann, Paris, 1992), vol. 2, p. 155.

[14] L. Blanchet, in Relativistic Gravitation and Gravitational Radiation, edited by J.-A. Marck and J.-P. Lasota, Les Houches School of Physics (Cambridge University Press, Cambridge, 1997), p. 33.

[15] R. Arnowitt, S. Deser, and C. W. Misner, in Gravitation: An Introduction to Current Research, edited by L. Witten (Wiley, New York, 1962), chap. 7.

[16] D. D. Holm, Physica 17D, 1 (1985). 